\title{
Mechanisms underlying the CNS myelination: A molecular and morphological analysis of the wrapping process
}

\author{
PhD Thesis \\ in partial fulfilment of the requirements \\ for the degree "Doctor of Philosophy" (PhD) \\ in the Neuroscience Program \\ at the Georg August University Göttingen, \\ Faculty of Biology
}

Submitter by

Nicolas Snaidero

Born in

Versailles, France

Göttingen 2014 
Members of the Thesis Committee:

Prof. Dr. Mikael Simons

Max Planck Institut für Experimentel Medizin

Department of Neurology, University of Gottingen

Prof. Dr. Uwe-Karsten Hanisch

Universitätsmedizin Göttingen Georg-August Universität Institut für Neuropathologie

Prof. Dr. Holger Stark

Max-Planck-Institut für Biophysikalische Chemie

AG 3D Kryo-Elektronenmikroskopie 


\section{Affidavit}

I hereby declare that this PhD thesis "Mechanisms underlying the CNS myelination; a molecular and morphological analysis of the wrapping process" has been written independently with no other aids or sources than quoted.

Nicolas Snaidero

January, 2014

Gottingen, Germany 


\section{Contents}

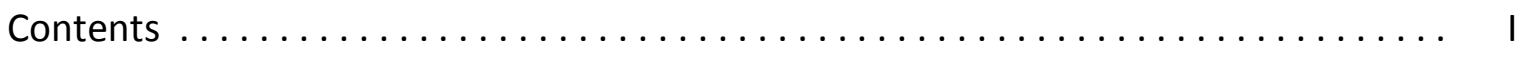

List of figures $\ldots \ldots \ldots \ldots \ldots \ldots \ldots \ldots \ldots \ldots \ldots \ldots \ldots \ldots \ldots \ldots \ldots \ldots, \quad V$

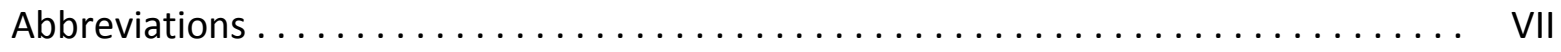

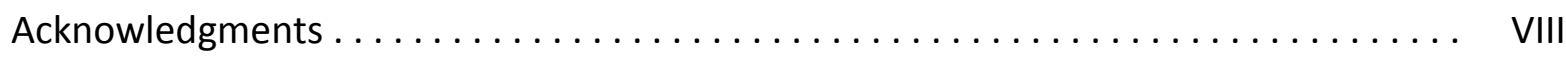

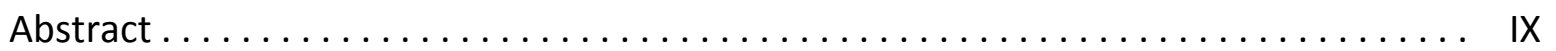

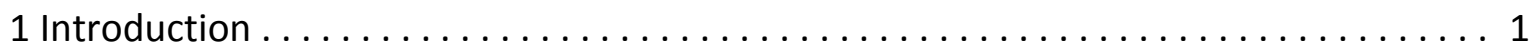

1.1 Organization of the nervous system in mammalians ................ 1

1.2 Cells constituting the nervous system $\ldots \ldots \ldots \ldots \ldots \ldots \ldots \ldots \ldots \ldots \ldots$

1.3 The development of myelin in evolution $\ldots \ldots \ldots \ldots \ldots \ldots \ldots \ldots \ldots$

1.4 Lineage, structure and function of the myelin sheath .............. 4

1.4.1 Lineage of the myelin forming cells .................. 4

1.4.2 Formation and appearance of myelin sheaths ............ 5

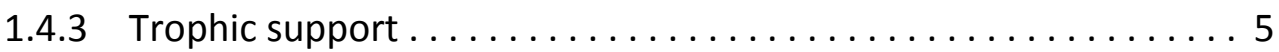

1.5 Composition of the CNS and PNS myelin sheath. ................ 6

1.6 Central regulators of cell polarity and cell growth in oligodendrocyte ..... 8

1.7 Mechanistic concepts of myelination $\ldots \ldots \ldots \ldots \ldots \ldots \ldots \ldots \ldots$

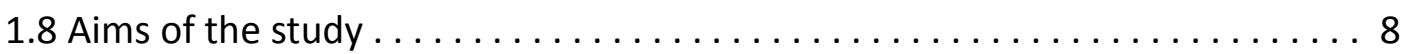

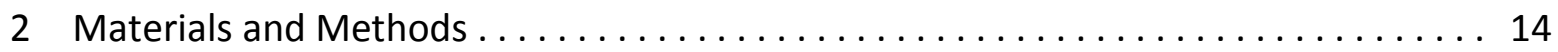

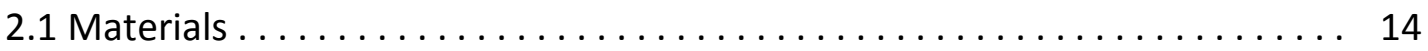

2.1.1 Equipment for electron microscopy .................. 14

2.1.2 Equipment for light microscopy ..................... 14

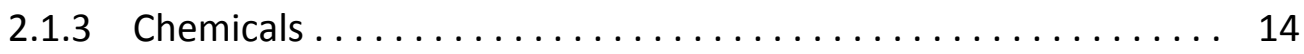

2.1.3.1 For electron microscopy .................. 14

2.1.3.2 For cell culture .......................... 15

2.1.3.3 Inhibitors for in vitro studies . . . . . . . . . . . . . 15

2.1.4 Plasmid .................................... 16

2.1.5 Animal lines................................. 16

2.1.6 Antibodies (primary and secondary) $\ldots \ldots \ldots \ldots \ldots \ldots \ldots \ldots$

2.1.6.1 Primary.............................. 16 
2.1.6.2 Secondary. ...................... 16

2.1 .7 Buffer list......................... 17

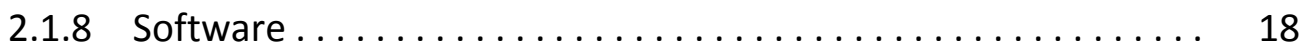

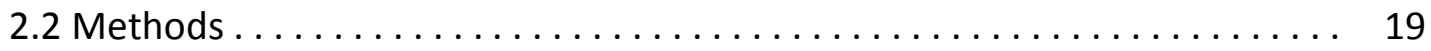

2.2.1 Cell culture techniques ...................... 19

2.2.1.1 Primary oligodendrocyte cultures . . . . . . . . . . . 19

2.2.1.2 Primary oligodendrocyte cells transfection ......... . 19

2.2.1.3 Chemical treatment of the Primary ........... 20 oligodendrocyte cultures

2.2.1.4 Infection of the Primary oligodendrocyte cultures by . . . 20 vesicular stomatitis virus (VSV)

2.2.1.5 Electrical stimulation of Primary oligodendrocyte cultures 20

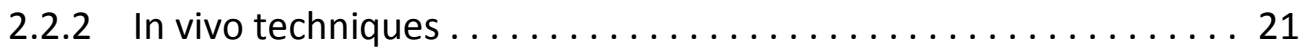

2.2.2.1 Live imaging and electron microscopy of myelination . . . 21 in Zebrafish

2.2.2.2 Mice handling and mutants $\ldots \ldots \ldots \ldots \ldots \ldots \ldots \ldots 22$

2.2.2.3 Sample preparation of mouse central nervous . . . . . . 22 system tissues

2.2.2.4 Intracerebral injection of VSV in Corpus callosum . . . . . . . 22

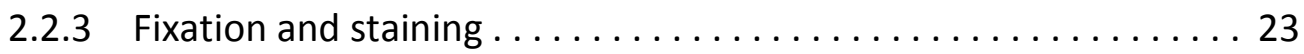

2.2.3.1 Chemical fixation and perfusion ............ 23

2.2.3.2 High pressure freezing fixation . . . . . . . . . . . 24

2.2.3.3 Immunocytochemistry . . . . . . . . . . . . . 24

2.2.3.4 Immuno-electron microscopy staining . . . . . . . 25

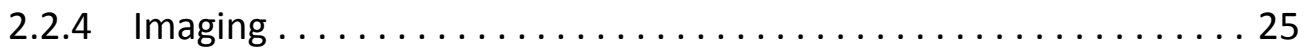

2.2.4.1 Transmission electron microscopy . ............. 25

2.2.4.2 Serial focus ion beam milling / scanning . . . . . . . 26 electron microscopy

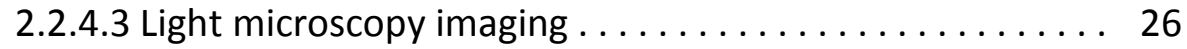

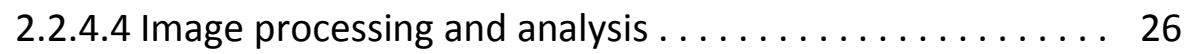

2.2.4.4.1 Transmission electron microscopy ........ 26 image analysis

2.2.4.4.2 Cultured oligodendrocyte cell rim ........ 27 quantification and surface VSV-G distribution

2.2.4.4.3 Zebrafish quantification ............ 27

2.2.4.4.4 3D segmentations of the FIBSEM data . . . . . 28

2.2.4.4.5 Immuno-electron microscopy labeling ..... 28 


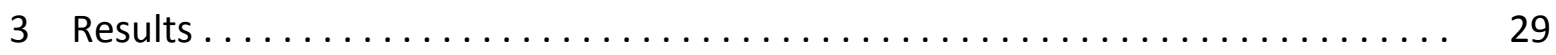

3.1 Morphology of the growing myelin sheath in vivo . . . . . . . . . 29

3.1.1 Three dimensional reconstructions of growing myelin . . . . . 29 sheaths by FIB-SEM

3.1.1.1 Technical approach to image a large volume ........ 29 at nanometer resolution range

3.1.1.2 Three dimensional visualization of myelination . . . . . 30 events at high resolution

3.1.2 Investigation of the myelin layer organization in the ..... 36 growing myelin sheath

3.1.3 In vivo motion of the growing myelin layers by . . . . . . . 40 live imaging in Zebrafish

3.2 Localization and characterization of the oligodendrocyte/myelin . . . . . . . 44 growth zone in vitro and in vivo.

3.2.1 Tracking the plasma membrane trafficking in cultured ...... 44 oligodendrocytes using the vesicular stomatitis virus

3.2.2 Subcellular localization of the VSV-G in growing myelin . . . . 50 sheath in vivo

3.3 Characterization of the mechanisms responsible for the polarized . . . . . 53 growth in oligodendrocytes.

3.3.1 Investigation of the oligodendrocyte inner tongue and ... . 53 outer rim during myelination.

3.3.1.1 Cytoskeleton distribution at the oligodendrocyte ..... 53 outer rim and inner tongue

3.3.1.2 Morphological evolution of the inner tongue . . . . . . 57 in vivo during myelin growth

3.3.2 Oligodendrocytic cytoplasm polarization during the myelin growth 58

3.3.2.1 The PI3K/AKT/mTOR signaling pathway enriched . . . . 59 at the leading edge of the oligodendrocyte

3.3.2.2 Regulation of the oligodendrocyte growth by ....... . 61 $\mathrm{PI3K} / \mathrm{AKT} / \mathrm{mTOR}$ signaling pathway

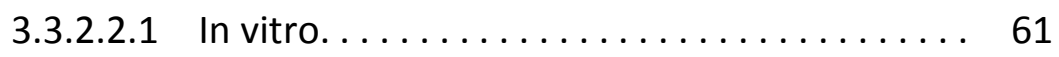

3.3.2.2.2 In vivo. ..................... 61

3.3.3 Artificial polarization of the oligodendrocyte cell trafficking . . . 64 by electrical stimulation

3.4 Myelin associated features in vivo. . . . . . . . . . . . . 66 
3.4.1 Cytoplasmic channels provide a short cut for transport . . . . . 66 towards the leading edge

3.4.2 Vesicular distribution along the non-compacted areas of ..... 69 growing sheaths

3.4.3 Redundant myelin during the myelin biogenesis . . . . . . . 71

3.4.4 MBP mediated compaction during the myelin growth . . . . . 74

3.4.4.1 Gradient of compaction within the growing myelin sheath 74

3.4.4.2 Growth and the compaction are independent processes . . 76

in the early stages of the myelination

3.4.4.3 Regulation of the compaction in the growing myelin sheath 77

3.5 Myelin sheath an adaptive and plastic structure

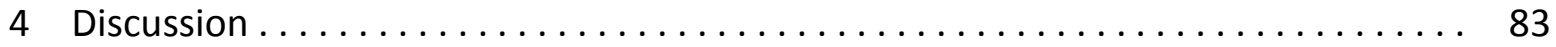

4.1 Tools for high resolution morphological analysis . . . . . . . . . . . . 84

4.2 Morphology of the growing myelin sheath in mammalian CNS . . . . . . 84

4.3 Identification of the leading edge in myelin $\ldots \ldots \ldots \ldots \ldots \ldots \ldots$

4.4 Central regulators of myelin growth $\ldots \ldots \ldots \ldots \ldots \ldots \ldots \ldots \ldots \ldots \ldots \ldots \ldots \ldots$

4.5 The cytoplasmic channels: a direct track from the cell body to . . . . . . . 91 the leading edge

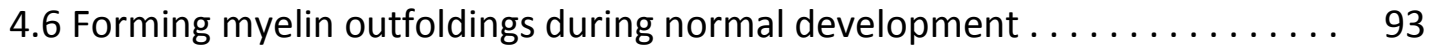

4.7 Sheath compaction proceed from the outer myelin layers . . . . . . . . 95

4.8 Axo-glia communication during myelination process $\ldots \ldots \ldots \ldots \ldots \ldots$

5 Summary $\ldots \ldots \ldots \ldots \ldots \ldots \ldots \ldots \ldots \ldots \ldots \ldots \ldots \ldots \ldots \ldots \ldots \ldots \ldots \ldots \ldots$

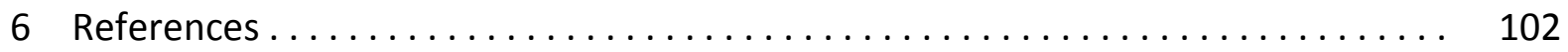

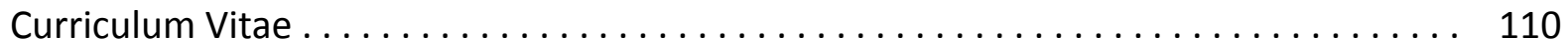

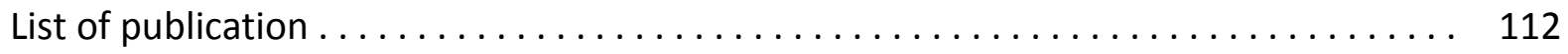




\section{List of Figures}

Introduction

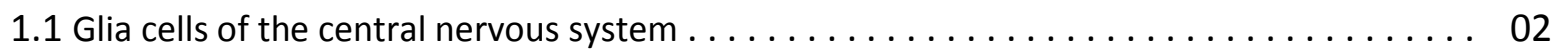

1.2 Ultrastructure of the myelin sheath in the CNS and PNS . . . . . . . . . . . 05

1.3 Distribution of the major CNS protein between compacted and non-compacted areas . . 07

Materials and methods

2.1 In vitro model of myelination: the primary oligodendrocyte cell culture . . . . . . . . . 19

2.2 Setup for cultured oligodendrocyte electrical stimulation $\ldots \ldots \ldots \ldots \ldots \ldots \ldots \ldots 21$

2.3 Myelin morphology preservation by high pressure freezing $\ldots \ldots \ldots \ldots \ldots \ldots \ldots$

Results

3.1 Sequential sample milling and imaging position during block face imaging $\ldots \ldots \ldots \ldots 31$

3.2 Resolution and cellular ultrasructure assessable by FIBSEM imaging $\ldots \ldots \ldots \ldots \ldots 2$

3.3 Three dimension high resolution investigation of myelin biogenesis $\ldots \ldots \ldots \ldots \ldots \ldots 32$

3.4 Investigation of the myelin ultrastructure of a growing sheath $\ldots \ldots \ldots \ldots \ldots \ldots \ldots$

3.5 Positioning of the inner and outer tongue along the growing myelinating sheath $\ldots \ldots 35$

3.6 Positioning of the successive myelin layers in a growing myelin sheath by TEM $\ldots \ldots \ldots 36$

3.7 Evolution of the internode morphology during CNS myelination $\ldots \ldots \ldots \ldots \ldots \ldots \ldots$

3.8 Evolution of the node morphology during CNS myelination $\ldots \ldots \ldots \ldots \ldots \ldots \ldots$

3.9 Organization of the successive myelin layers along a full internode during myelination . . . 39

3.10 Live imaging of myelination events in Zebrafish $\ldots \ldots \ldots \ldots \ldots \ldots \ldots \ldots \ldots \ldots$

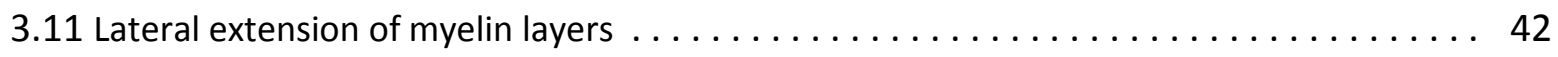

3.12 Tracking the membrane trafficking using the vesicular stomatitis virus $\mathrm{G}$ protein $\ldots \ldots 45$ in oligodendrocyte cell culture.

3.13 Subcellular localization of the VSV-G in cultured oligodendrocyte $\ldots \ldots \ldots \ldots \ldots$

3.14 Features of the VSV-G distribution on cultured oligodendrocyte $\ldots \ldots \ldots \ldots \ldots \ldots$

3.15 VSV particles localization in cell culture $\ldots \ldots \ldots \ldots \ldots \ldots \ldots \ldots \ldots \ldots \ldots \ldots$

3.16 Tracking the membrane trafficking using the vesicular stomatitis virus $\mathrm{G}$ protein . . . . . 51 in vivo during myelination

3.17 Tracking the membrane trafficking using the vesicular stomatitis virus $G$ protein . . . . . 52 in vivo after completion of myelination

3.18 Evolution of the oligodendrocyte morphology upon development in culture . . . . . . . 54

3.19 Localization of the F-actin cytoskeleton in vivo $\ldots \ldots \ldots \ldots \ldots \ldots \ldots \ldots \ldots \ldots \ldots \ldots \ldots \ldots \ldots$

3.20 Evolution of the morphology of the non-compacted areas of the myelin sheath $\ldots \ldots \ldots 58$ upon development in vivo 
3.21 Cytoplasmic polarization of the oligodendrocyte during the growth $\ldots \ldots \ldots \ldots \ldots$

3.22 Inhibition of the PI3K pathway alter the oligodendrocyte rim in vitro $\ldots \ldots \ldots \ldots 2$

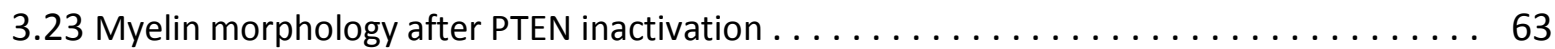

3.24 Artificial polarization of the cytoskeleton, PI3K signaling and plasma membrane trafficking 65

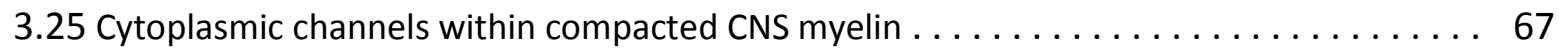

3.26 Three dimensional morphology of the cytoplasmic channels . . . . . . . . . . . 68

3.27 Vehicular profiles distribution within the myelin sheath during its maturation . . . . . 70

3.28 Myelin outfoldings in the developing myelin sheath $\ldots \ldots \ldots \ldots \ldots \ldots \ldots \ldots \ldots$

3.29 Three dimensional view on the outfoldings $\ldots \ldots \ldots \ldots \ldots \ldots \ldots \ldots \ldots \ldots \ldots \ldots$

3.30 Compaction of the growing myelin sheath $\ldots \ldots \ldots \ldots \ldots \ldots \ldots \ldots \ldots \ldots \ldots \ldots \ldots \ldots \ldots \ldots$

3.31 Effects of MBP levels on compaction and myelin stability $\ldots \ldots \ldots \ldots \ldots \ldots \ldots \ldots$

$3.32 \mathrm{CNP}$ a possible regulator of the myelin compaction $\ldots \ldots \ldots \ldots \ldots \ldots \ldots \ldots \ldots$

3.33 Interconnection between the cytoplasmic channels and the myelin growth . . . . . . 82

\section{Discussion}

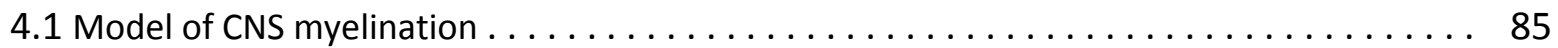

4.2 Lateral extension of the myelin layers during development $\ldots \ldots \ldots \ldots \ldots \ldots \ldots \ldots 88$

4.3 Model of outfolding formation and resolution during myelin growth . . . . . . . . 94

4.4 Model of the myelin morphology during growth and MBP compaction . . . . . . . . . . . 97 


\section{Abbreviations}

CC: Corpus Callosum

CNP: 2',3'-cyclic nucleotide 3' phosphodiesterase

CNS: Central Nervous System

DIV: Day In Vitro

EM: Electron Microscopy

FIBSEM: Focus Ion Beam Scanning Electron Microscopy

GFP: Green Fluorescence Protein

MAG: Myelin Associated Glycoprotein

MBP: Myelin Basic Protein

MIA: Multiple Image Acquisition

MOG: Myelin Oligodendrocyte Glycoprotein

OPC: oligodendrocyte precursor cells

$\mathrm{PO}$ :Peripheral myelin Protein 0

$\mathrm{P} 2$ :Peripheral myelin Protein 2

PBS: Phosphate buffered saline

PLP: Proteolipid Protein

PMP-22: Peripheral myelin protein 22

PNS: Peripheral Nervous System

PTEN:Phosphatase and Tensin homolog

SEM: Scanning Electron Microscopy

TEM: Transmission Electron Microscopy

VSV: Vesicular Stomatitis Virus

YFP: Yellow Fluorescence Protein 


\section{Acknowledgments}

I would like to thank my supervisor Mikael Simons for supporting and guiding me during this $\mathrm{PhD}$ and for offering the opportunity to be part of so many exiting projects. Thank you Mika, for your enthusiasm and trust.

I want to express my most sincere gratitude to Michael Hörner and Sandra Drübe from the IMPRS Neuroscience coordination office who believed in me and offered me the chance to be part of this program but also for their constant support throughout all these years.

I also want to thank Dr. Wiebke Möbius for introducing me to the Electron microscopy world and for being very supportive scientifically and personally and also Prof. Klaus Armin Nave for

I thank Prof. Dr. Uwe-Karsten Hanisch and Prof. Dr. Holger Stark for the fruitful discussions as members of my PhD thesis committee.

I also appreciate all the help from our collaborators: Wiebke Möbius, Tim Czopka, David A. Lyons, Klaus Armin Nave, Sandra Göbbles, Julia Edgar, Doron Merkler, and at the FEI Company: Liesbeth H.P. Hekking, Cliff Mathisen and Dick Verkleij.

I would like to mention the colleagues and friends in AG Simons, past and present who provided a nice and friendly working environment: Giselheid Schulz, Larisa Yurlova, Lena Steshenko, Dirk Fitzner, Aniket Ghosh, Natalia Manrique Hoyos, Mostafa Bakhti, Sebastian Schmitt, Shweta Aggarwal, Tina Kling, Shima Safaiyan, Schanila Nawaz, Nils Halbsgut, MarieTheres Weil, Caroline Velte and Ludovico Cantuti.

My thanks to Torben Ruwedel for EM technical support and for a lot of fun time throughout all these years and to Giselheid Schulz for her technical support concerning the cell culture.

Special thanks to Mosi for our "philosophical" discussions and for understanding and accepting me as I am.

Also my dear friend Pierre Emmanuel who was always there when needed for support and fun.

My parents for their unconditional support and love in all the steps of my life especially these past years of doctoral studies.

I want to thanks my dear Sasha for sharing all the good and bad moments with me and for her bulletproof patience and humongous support scientifically and everywhere else, making all this possible. 


\section{Abstract}

Central nervous system myelin is a multilayered membrane sheath generated by oligodendrocytes for rapid axonal electrical impulse propagation. Both light and electron microscopy have been used to describe morphological features of the myelin sheath. However, the underlying mechanisms of myelin wrapping are still unclear and remain under debate. To investigate the morphology of the CNS myelin sheath during its formation we made use of multiple imaging techniques such as focus ion beam milling coupled to scanning electron microscopy, live imaging, transmission electron microscopy, cryo-immuno labeling and confocal microscopy. With these various approaches we could reconstruct the growing myelin sheath structure in three dimensions and obtain a dynamical view of the myelination. Furthermore, using mouse genetics and virus as reporter we could identify the growth front of the myelin at the inner tongue of the sheath, which is under the control of the PI3K pathway.

We identified an elaborated system of cytoplasmic channels within the growing myelin sheath, which serves as tracks for membrane trafficking to the leading edge at the inner most tongue. Most of these channels disappear with the maturation of the sheath but can re-open in the adult when phosphatidylinositol-(3,4,5)-triphosphate was experimentally raised.

Taken together, our model suggests that a growing myelin sheath winds around the axon by an advancing inner tongue (underneath the previously deposited membrane) in the center of the myelin segment. Concurrent to this radial growth of the sheath the layers extend laterally towards the node of Ranvier. Our model can explain how myelin is formed as a multilayered structure in the CNS, how abnormal myelin outfoldings are generated in neurological disease and the plasticity of myelin biogenesis in adult life. 


\section{INTRODUCTION}

\subsection{Organization of the nervous system in mammals}

The nervous system is the most complex organ in vertebrates coordinating voluntary and involuntary actions and allowing the processing and transmission of signals from external and internal inputs in order to execute a response to them.

The mammalian nervous system is composed of two distinct morphological and functional entities that are the central nervous system (CNS), that includes the brain and the spinal cord and the peripheral nervous system (PNS), that contents the cranial nerves, spinal nerves and connections between the CNS and the target organs of the body.

CNS is a control center that integrates all input signals from PNS and CNS to evoke an appropriate response. PNS, on the other hand, provides the sensory and motor wiring to convey and elicit the answers to the rest of the body in a voluntary (somatic nervous system) or involuntary modality (autonomic nervous system) (Kandel et al., 2000).

\subsection{Cells constituting the nervous system}

The cells constituting the nervous system belong to two main classes: primarily the neurons that can illicit an electrical impulse to be transmitted to another neuron or to other cells types by synaptic communication. The second type is the supportive glial cells that represent the majority of the cells in the mammalian CNS and belong to three main categories: the microglia, the astrocytes and the myelin forming cells (oligodendrocytes in the CNS and Schwann cells in the PNS) (fig 1.1). Each of these cell types performs different supportive tasks in the central nervous system. The microglial cells are the resident immune cells of the nervous system providing defense against pathogens and being responsible for the clearance of dead cells (Graeber et al., 2011). The astrocytes form the blood-brain barrier that separates the CNS from the systemic circulation, but they also support neurons by providing energy and specific substrates and acting as a recycling partner of the synapses (Allen and Barres, 2009) (fig 1.1). 
The myelin forming cells like Schwann cells and oligodendrocytes generate the myelin sheath of the axon allowing the fast saltatory conduction of the action potential along the axon, drastically increasing the speed of its propagation in function of the diameter of the axon (Hartline and Colman, 2007). In addition to the insulating function, the myelin sheath provides trophic support to the axon during all its life (Nave and Trapp, 2008).

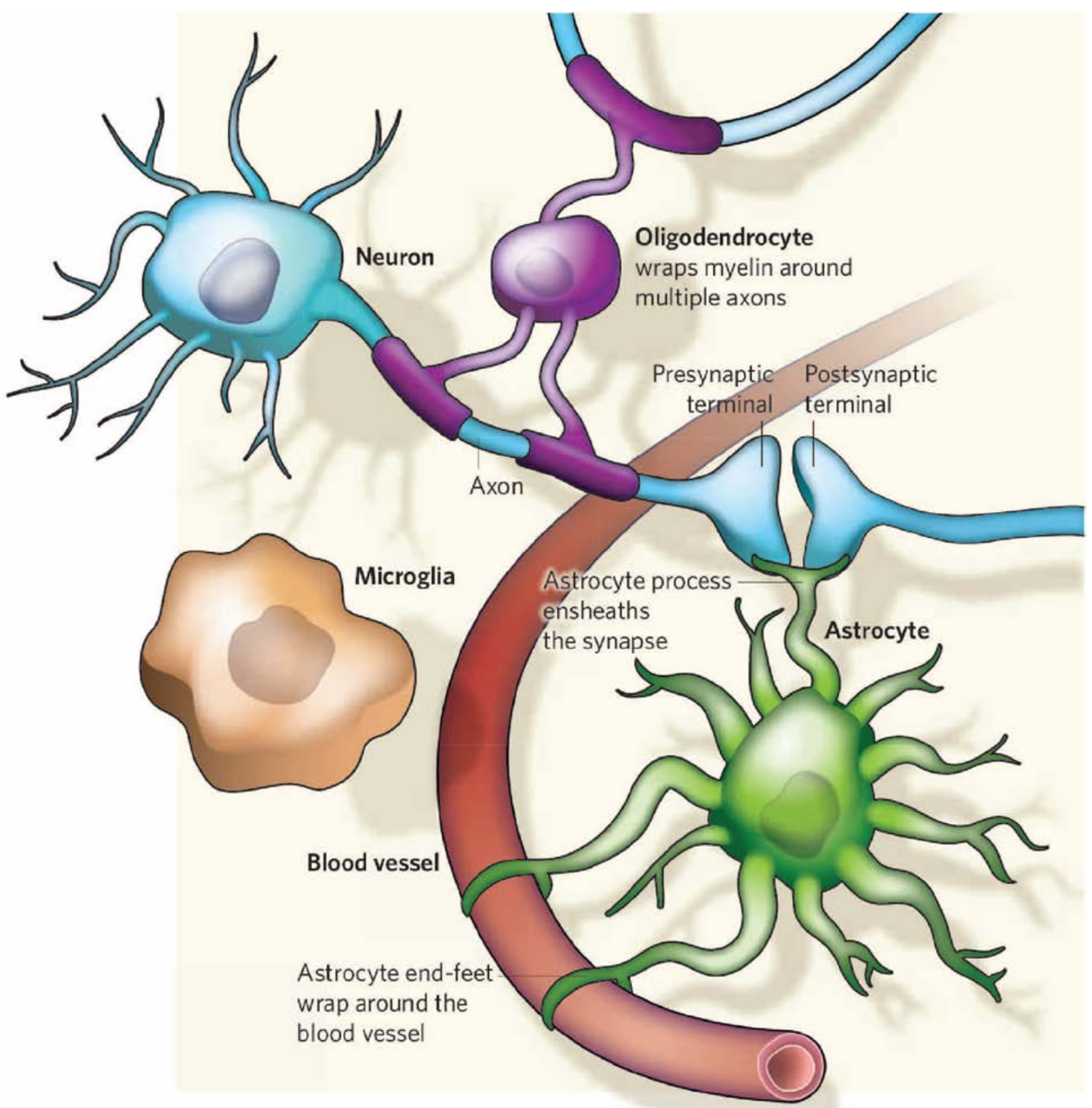

Figure 1.1: Glia cells of the central nervous system

Drawing of the glia cell types in the central nervous system showing their relation with the neurons, synapses and blood vessels. Figure from Allen and Barres, Nature 2009. Nature publishing group license Number 3314160956305. 


\subsection{The development of myelin in evolution}

During evolution, different organisms have expressed a need to increase the electrical impulse conduction speed. Two mechanisms are possible to reach this goal. The first is an increase in axon diameter leading to a decrease in the interior resistance and thus a faster axon potential propagation. The second is a decrease in the trans-fiber capacitance that is achieved by the development of myelin sheath segments around the axon.

Without compact myelin sheath the average action potential propagation speed is around $1 \mathrm{~m} / \mathrm{s}$. Based on these properties most of the invertebrates that are below 30 centimeters of size could survive without myelin. Some exceptions among invertebrates, such as cephalopods (including giant squids), can reach up to several meters long; to compensate the lack of myelin

sheath these species have developed giant axons that allowed a faster propagation of the action potential (Zalc, 2006).

In most of the vertebrates another approach developed over time: the compact myelin sheath. The myelin sheath surrounds axons by successive segments leading to an increase of action potential speed by 50 to 100 folds compared to the average action potential speed found in the invertebrates (Hartline and Colman, 2007).

When comparing these two approaches to increase the axon conduction speed, it appeared that the sheath of myelin was far more beneficial as it required thousand times less energy and space and thus is more appropriate for larger and more complex organisms (Hartline and Colman, 2007); (Quarles, 2002).

\subsection{Lineage, structure and function of the myelin sheath}

\subsubsection{Linage of the myelin forming cells}

In the CNS, the oligodendrocyte precursor cells (OPCs) are derived from the neuroepithelium of the sub-ventricular zone and can differentiate into mature oligodendrocytes. OPCs can be identified by their expression of NG2, chondroitin sulphate proteoglycan or platelet-derived growth factor (Levine et al., 2001). Possibly upon axonal contact but also by cell-intrinsic mechanisms the cell transits to a myelinating state where the O4,GalC and PLP/DM20 markers start to be expressed (Levine et al., 2001). 
In the PNS the Schwann cell precursors arise from the neural crest cells leading to Schwann cell precursors, immature Schwann cells and myelin or non-myelin forming Schwann cells (Mirsky et al., 1996); (Jessen and Mirsky, 2005).

\subsubsection{Formation and appearance of myelin sheaths}

In the mouse central nervous system the myelination starts after birth, peaks at P20 and is almost over by P60 (Baumann and Pham-Dinh, 2001); (Vincze et al., 2008). In humans myelination starts during mid-gestation and is largely ended at 40 months postnatal but can proceed unto young adulthood (Parazzini et al., 2002). Some oligodendrocytes can produce only few myelin sheaths at a time whereas others are able to myelinate up to 50 internodes in other regions (Peters and Proskauer, 1969); (Remahl and Hilderbrand, 1990). The oligodendrocytes start to myelinate axons with a diameter of at least $300 \mathrm{~nm}$ (Almeida et al., 2011); (Lee et al., 2012) and extend laterally along the axon for 150-300 $\mu \mathrm{m}$ (Ransom et al., 1991) (fig 1.2).

In the peripheral nervous system the myelin sheath is produced by the Schwann cells that myelinate only one internode. Only the axons larger than 1 micrometer of diameter are myelinated (Sherman and Brophy, 2005), while the smaller ones are surrounded by nonmyelinating Schwann cells. The internodal length in the PNS can reach up to $1 \mathrm{~mm}$ (Jaros and Jenkison, 1983) with up to 300 concentric layers (fig 1.2).

The myelin sheaths produced in the CNS and the PNS have defined thickness for a given axon diameter and the ratio of the myelin volume reported to the axon volume (G-Ratio) is, in normal condition, around 0,6 to 0,7 to insure optimal saltatory signal propagation (Rushton, 1951); (Friede and Miyagishi, 1972); (Chomiak and Hu, 2009).

\subsubsection{Trophic support}

In addition to providing the saltatory conduction of the neuronal electrical Impulse the myelin has lately been described to provide a trophic support to the axons along their life (Nave, 2010). Deletions of oligodendrocyte specific proteins have been shown to be responsible for axonal pathologies and degeneration even in the absence of obvious morphological defects in 
A Central nervous system
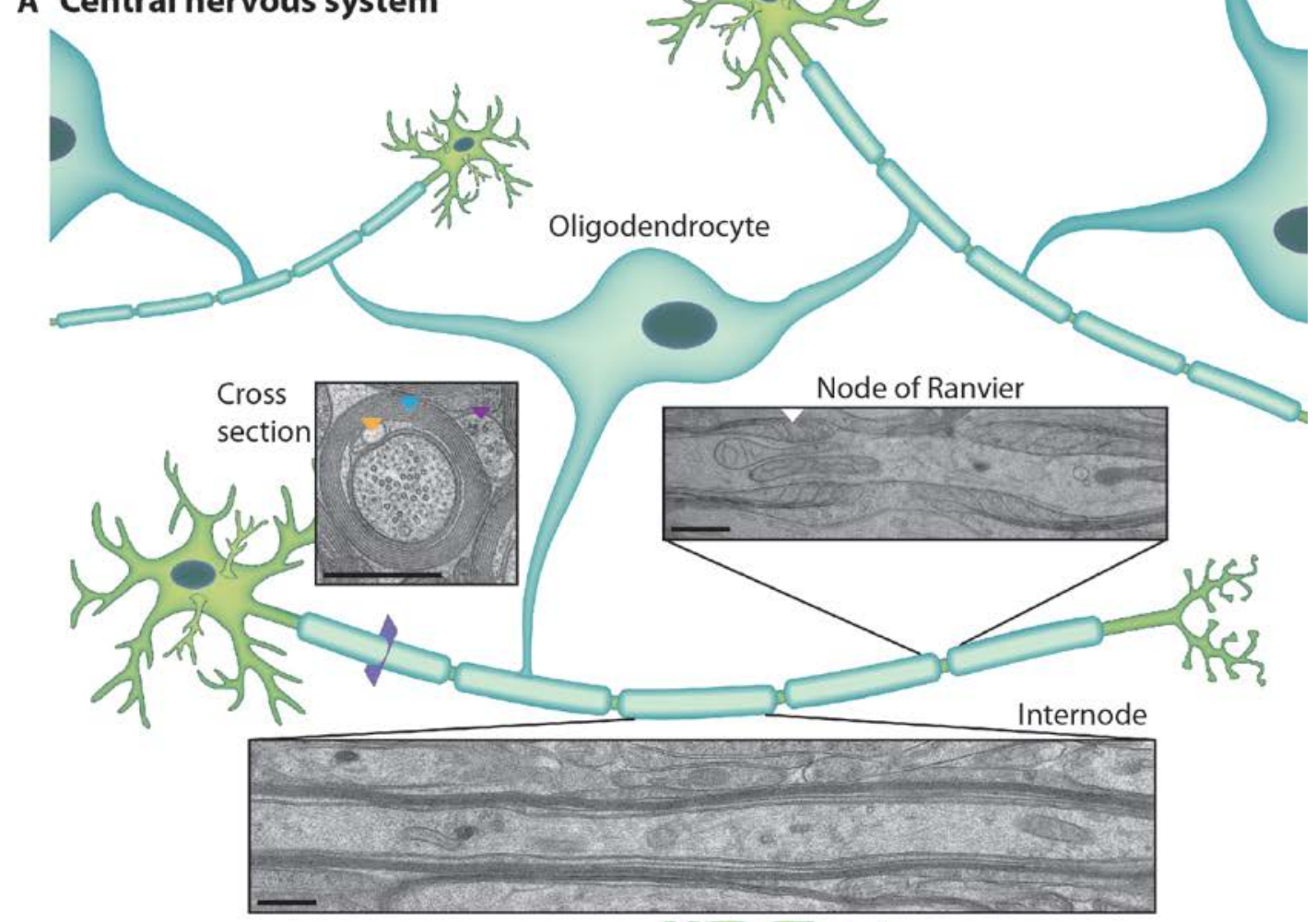

B Peripheral nervous system

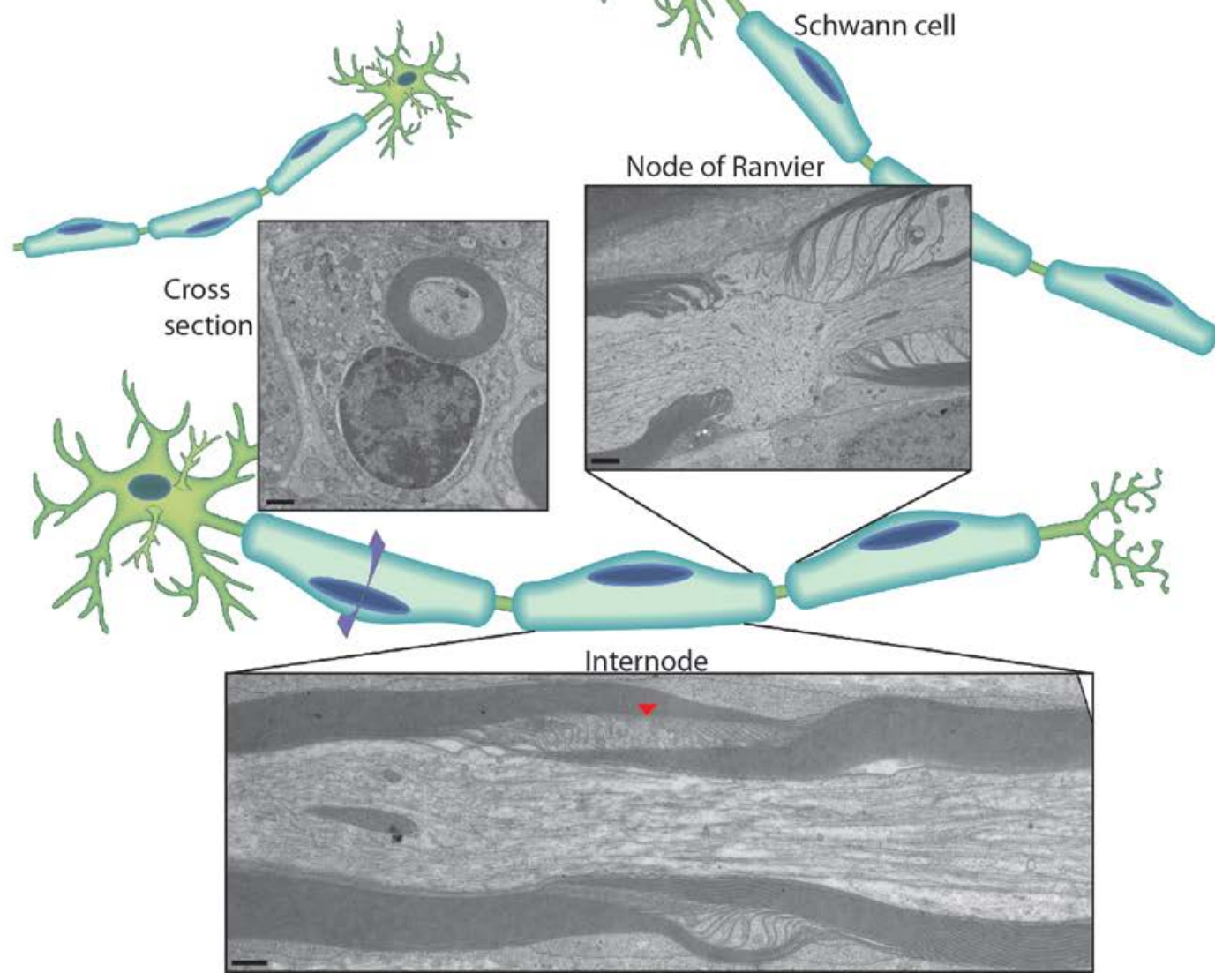




\section{Figure 1.2: Ultrastructure of the myelin sheath in the CNS and PNS}

A) The oligodendrocyte cell body is situated away from the axons and it can myelinate several internodes at a time. In a cross sectional view the following domains of the myelin sheath are present non-compacted inner tongue in contact with the axon (orange arrow), the spiral of tightly compacted layers (cyan arrow) and the non-compacted outer tongue (purple arrow). At the node of Ranvier these two non-compacted compartments are in contact via the paranodal loops (white arrows). Along the internode the myelin layers extend uninterrupted along the axon. B) The Schwann cells are in direct contact with the axon, producing one myelin segment per cell. The cytoplasm of the cell body can be seen all along the internode (stars) surrounding the compacted spiral of membrane (cyan arrow). The inner tongue is also non-compacted and in direct contact with the axon. The myelin sheath is generally thicker in the PNS and large cytoplasmic openings can be seen along the internode: the Schmitt Lanterman incisures (red arrow). Scale bar $500 \mathrm{~nm}$.

the sheath itself (Griffiths et al., 1998), (Lappe-Siefke et al., 2003). It has been proposed that the oligodendrocyte may provide metabolic support to the long axons by providing lactate that would then be used by the axons to support their mitochondrial energy metabolism (Funfschilling et al., 2012); (Lee et al., 2012); (Saab et al., 2013).

\subsection{Composition of the CNS and PNS myelin sheath}

The myelin sheath in mammals is a structure that presents a unique composition with low water content of only $40 \%$ and a high proportion of lipids with $70-85 \%$ of the dry mass. Consequently, the amount of protein in this structure is particularly low compared to normal membrane composition with only $15-30 \%$ of the dry mass (Waxman et al. 1995). The lipid composition of the myelin sheath is not unique but the cerebrosides (galactosylceramide derivatives) are found especially in this structure. Most of the remaining defining lipids of the myelin are sulfatides, cholesterol and plasmalogens. The PNS and CNS have very similar lipids constituting the myelin sheath, but with a different ratio (Waxman et all 1995).

However the protein composition of the CNS and PNS myelin varies a lot. Few major proteins constitute the CNS myelin, such as Myelin Basic Protein (MBP) and Proteolipid Protein (PLP) that would count for $60-80$ percent of the total protein content of the sheaths. Additional proteins, such as Myelin-Associated Glicoprotein (MAG), 2',3'-cyclic nucleotide 3' phosphodiesterase (CNP), and Myelin Oligodendrocyte Glycoprotein (MOG) count for most of the remaining proteins (fig 1.3). 
The PNS myelin is constituted by a different set of proteins which, however, leads to a similar structure. The major proteins that can be found in the PNS are P0, PMP-22, P2, MBP and MAG proteins (Quarles et al., 2006). In addition to the main myelin components listed above, there is a growing list of proteins that are found mainly by proteome analysis (Werner et al., 2007).

B

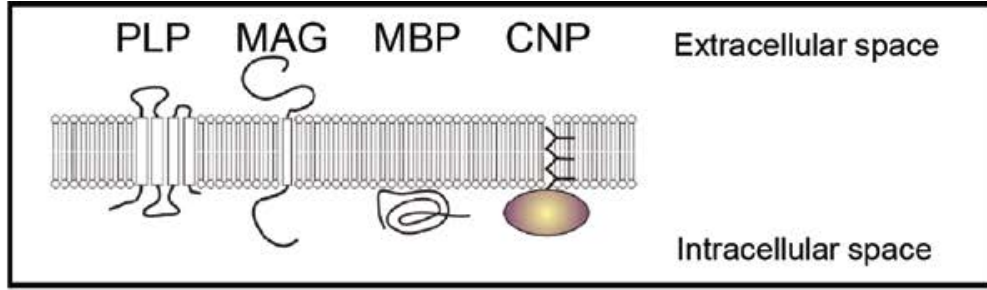

A

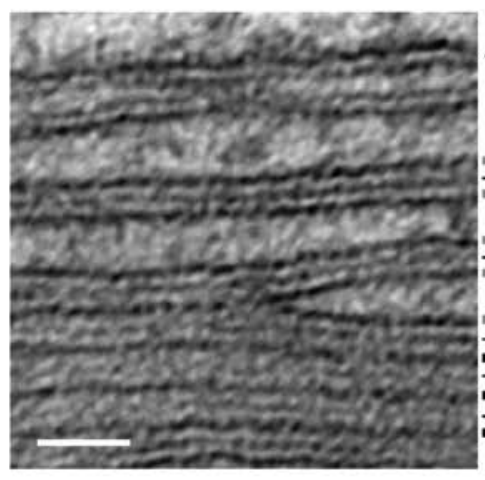

$\leftarrow$ Axolema

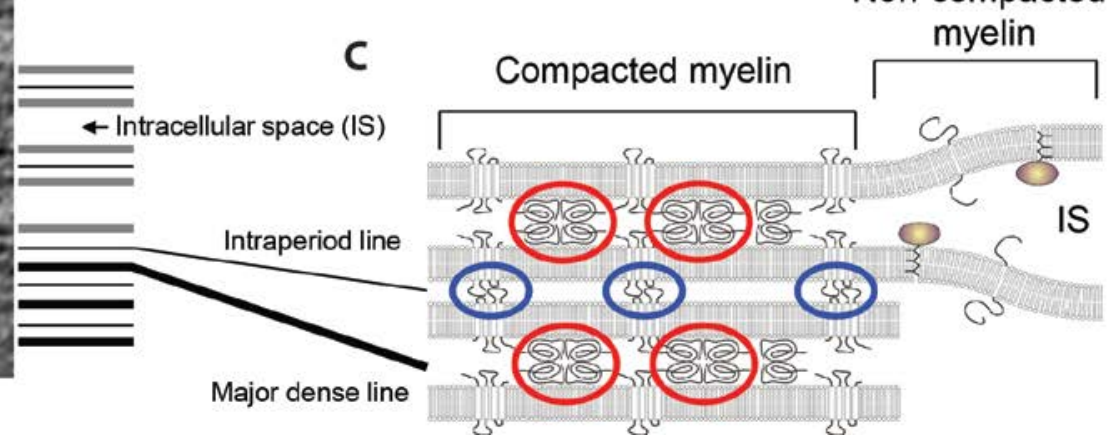

Figure 1.3: Distribution of the major CNS proteins between compacted and non-compacted areas

A) High magnification of a CNS myelin electron micrograph. Scale bar $25 \mathrm{~nm}$. B, C) Schematics representation of the proteins responsible for the intracellular compaction (MBP, circled in red) and involved in the extracellular compaction (PLP, circled in blue) as well as the segregation of the proteins with large intra and extracellular domain to the non-compacted areas of the sheath. 


\subsection{Central regulators of cell polarity and cell growth in oligodendrocyte}

The massive remodeling of the oligodendrocyte during myelination requires a cellular polarization to direct the growth towards the tip of the forming myelin sheath.

The machinery needed to polarize cell growth into one direction is extremely complex and may vary between cell types (Arimura and Kaibuchi, 2007); (Martin-Belmonte and RodriguezFraticelli, 2009). However, one key pathway is the PI3K/Akt signaling cascade, which regulates growth at the leading edge in most cells (Wullschleger et al., 2006); (Arimura and Kaibuchi, 2007); (Manning and Cantley, 2007); (Laplante and Sabatini, 2009); (Ridley, 2011). The PI3kinase and mTOR pathways are the central signaling axis that has been described to be involved in a very large and diverse group of cellular functions, including cell growth, motility, survival, proliferation and intracellular trafficking.

The oligodendrocytes also make use of this pathway even if they are non-migratory (Taveggia et al., 2010). The up-regulation of the PI3K/Akt/mTor pathway was shown to enhance myelination and even to lead to the pathological outgrowth of myelin (Flores et al., 2008); (Goebbels et al., 2010); (Goebbels et al., 2012) while its down-regulation would lead to the opposite effect (Narayanan et al., 2009).

\subsection{Mechanistic concepts of myelination}

Subsequently after the identification of the neuroglia surrounding neurons and axons, Del RioHortega described several glia cell types including oligodendrocytes in the vicinity of axons which were covered by the myelin sheath. The term "myelin" was coined by Wirchov already in the $18^{\text {th }}$ century without knowing from which cell-type it derived. Later it was proposed that oligodendrocytes are the cells that are producing the myelin sheath (Rosenbluth 1999). At that time the myelin sheath was thought to be a crystal-like structure derived from an accumulation of secreted material on the surface of the axon, but in 1954 Betty Ben, using transmission electron microscopy could show that the Schwann cells are directly connected to the myelin sheath (Geren and Schmitt, 1954).

Following this seminal discovery, scientists started to investigate the morphology of the myelin sheath in the PNS and then CNS to understand the architecture of this structure and its 
formation. Robertson and Engstrom could show that the PNS myelin sheath is composed of double membrane layers compacted and tightly wrapped around the axon (Robertson, 1955) and that the fusion of the two membrane leaflets would lead to the formation of the major dense line (Engstrom and Wersall, 1958).

The PNS myelin was the first to be investigated due to the relatively easy access, but soon the CNS myelin started to be studied as well. Indeed, in 1956 Sarah A. Luse observed that the CNS myelin, produced by oligodendrocytes in CNS, is based on the same principle architecture as PNS myelin, and also reported an increase of the amount of layers with maturation (Luse, 1956). From these observation a model of CNS myelination was proposed where the oligodendrocyte would produce membrane fragments that would be incorporated into the growing myelin around the axon. A similar concept of secreted myelin sheath was also proposed by Robertis (De Robertis et al., 1958). At the same time Peters started his morphological investigations on the PNS myelin by describing the Schwann cell during myelination and also characterized the myelinating and non-myelinating Schwann cells (Peters and Muir, 1959). By using a better sample preparation technique Robertson also investigated the morphology of the node of Ranvier (Robertson 1959). With the improvement of the sample preservation for TEM the focus was brought on the compacted and non-compacted areas of the myelin sheath during development leading to a better understanding of the overall morphology of the myelin sheath and to the identification of similarities and differences between CNS and PNS myelin sheaths (Maturana, 1960); (Peters, 1960a); (Peters, 1960b).

In 1961 Bunge and colleagues published a landmark study where they described the oligodendrocyte to be connected to the several myelin sheaths. They also proposed that the paranodal loops, outer and inner mesaxons (inner and outer tongue) would be connected together and form one continuous cytoplasmic compartment. Based on several electron micrographs this work proposed a growth model where the oligodendrocyte would surround the axon with one wrap covering the entire internodal length and would subsequently wrap as a spiral around the axon. It was also hypothesized that the inner mesaxon would be the advancing edge of the growing myelin sheath (Bunge et al., 1961). This model was later referred as the "carpet crawler model". 
Following the technical developments and the sample preparation improvements, qualitative investigations of the spacing between the successive layers were performed (Peters, 1962).

Studies on interaction between the axon, the oligodendrocyte and the myelin sheath could show that myelination occurs only on axons thicker than $300 \mathrm{~nm}$ in diameter. It was also demonstrated that the axon caliber would increase concomitantly with the myelination (Gyllensten and Malmfors, 1963); (Allt, 1969). Furthermore, it was reported that keeping mice in total darkness during development would delay myelination and axon caliber would increase in the optic nerve (Gyllensten and Malmfors, 1963). Finally, it was shown that the length of the oligodendrocytic process contacting the myelin sheaths could vary drastically reaching few $10^{\text {th }}$ of micrometers (Peters, 1964).

In an ultrastructure study of the CNS myelin Peters and colleagues identified the radial component (structural specialization within CNS myelin that is believed to stabilize the apposition of membranes in the internode) to be mainly located between the inner and outer mesaxons in adult CNS myelin. These scientists were also able to show that the inner and outer mesaxon are localized in the same quadrant around the axon. Interestingly, it was found that during myelin growth, the inner and outer mesaxons would be randomly positioned around the axon and that a clear correlation could be drawn between the amount of myelin lamella and the maturation state of the sheath (Peters, 1964). In another study they could identify the septate junctions at the nodal region as dark formations between the paranodal loops and the axon (Peters, 1966). Hirano and Dembitzer using a very high quality EM, could describe some "isolated island of cytoplasm" within the compacted myelin sheath, double myelination patterns and identified several organelles within cytoplasmic areas. The model they proposed was a refined view of the carpet crawler where the densification at the paranodal loops was explained and where an explanation for the non-canonical myelin profiles, such as double myelination or isolated island of cytoplasm, was proposed. However in this study the growth of the myelin was hypothesized to be happening from the outer layers of the sheath (Hirano et al., 1970). Until this point most of the studies were performed on isolated observations and conclusions were made based on the morphology of few profiles. Starting from 1970 several quantitative studies were performed on larger amounts of samples to monitor the variation of 
myelin amount in optic nerve during the development (Matheson, 1970); (Matthews and Duncan, 1971); (Moore et al., 1976).

Concomitant with the studies done on the CNS the morphology of the PNS was heavily studied and a better knowledge of the Schwann cell morphology was obtained leading Webster to characterize the Schmitt-Lanterman incisures and also to hypothesize that the inner mesaxon would be the motile part in the peripheral myelin (Webster, 1971).

A great technical improvement in the investigation of the myelin formation was achieved by Robert L. Knobler and colleagues by performing the first serial sections and reconstruction of the CNS myelin to understand its morphology in 3 dimensions. Within few micrometers of young rat spinal cord they could show that the amount of myelin wraps was not constant along the segments and that "vermicular processes" were extending within the forming sheaths. Based on these observations the authors hypothesized that several of these processes would wind around the axon like a "thread of a screw" and then flatten to give rise to plates of cytoplasm (Knobler et al., 1974); (Knobler et al., 1976).

Another very different model of myelination was proposed by Richard and colleagues in which based on the on the orientation of the mesaxons in the myelin sheath and the positioning of the oligodendrocytes, the cell body pulsation would contribute to axon wrapping by repetition of successive "wrap around" and "wind-up" (Richards et al., 1983).

However, by analyzing the movement of the cell body of the Schwann cells during m6elination, it became clear that the cell is stationary and does not rotate around the myelin sheath during myelination. This would suggest that the sheath is growing by the movement of the inner mesaxon (Bunge et al., 1989).

In 1988 Richard C. Wiggins and colleagues made a very interesting hypothesis based on a single observation when they were investigating the contact between the axon and the growing sheath. They observed the growing the myelin sheath with a gradient of compaction and postulated that the growth could, in certain case, "outpace" the sheath compaction (Wiggins et al., 1988).

In the early ninety's it became apparent that studies on myelin morphogenesis would need to be performed on larger areas, volumes and sample numbers. In this perspective Remahl, 
Hildebrand, Berry and colleagues performed large studies on spinal cord serial sections where they could identify oligodendrocytes myelinating axons of similar diameters and producing myelin of similar thickness. Furthermore they described that different oligodendrocyte types would myelinate thin or thick axons with 1 cell contacting 15-30 small caliber axon and other cells forming myelin on 1-3 large axons (Berry et al., 1995). Moreover, when investigating the non-compacted areas of the growing myelin sheath it appeared that the orientation of the inner and outer mesaxon in the myelin sheath produced by a same glia cell would be random (Remahl and Hilderbrand, 1990). This observation would contradict the model proposed by Richards et al in 1983. Furthermore, using large volume of data they could identify the compaction state and the myelin thickness along myelinating axons. It was found found that the axons diameter would be larger in the portion where it was surrounded by myelin. Based on the positioning of the compacted and non-compacted myelin around the axons, they proposed that the sheath is elongating in a one wrap along the entire internode before starting the active wrapping and compaction (Remahl and Hildebrand, 1990). Based on the light microscopy investigation of injected dyes Berry and colleagues proposed that the sheath would be formed by spiral deposition of the outer tongue and that the layers close to the axon would grow laterally faster than the layers more superficial of the sheath (Berry et al., 1995).

Most of the studies were performed on wild type animals using mainly morphological analysis. However, when the area of mouse genetic started, several mouse mutants were generated followed by the characterization of myelin pathologies and aberrant formations of myelin sheaths.

In 2010 the question of how the myelin is formed was re-addressed using molecular tools and new techniques, although mainly based on light microscopy.

Based on the Caspr and NF155 protein distribution along the forming myelin sheath, Pedraza and colleagues proposed a model where a ribbon-like process of the oligodendrocyte would coil around the axon following the pattern of the caspr and NF155 proteins (Pedraza et al., 2009). With the help of cytoskeleton remodeling the processes would flatten and cover each other to reach a spiral formation at the mature stage, also reviewed in (Bauer and ffrench-Constant, 2009). This view was adapted in the work of loannidou and colleagues where several ribbons of 
membrane would contribute to the coil around the axon and would fuse to achieve a mature sheath (Ioannidou et al., 2012).

Later, Sobottka and colleagues proposed a model where the axon was suggested to be actively involved in the formation of the myelin sheath. Axon would pull the oligodendrocyte process around itself leading to a coiling of the inner and outer tongue. By live imaging the forming sheath was observed to be thicker in the middle and the outer tongue coiling was measured to be around 7,5 $\mu \mathrm{m}$ during the formation of the sheath (Sobottka et al., 2011).

To investigate the intrinsic myelination properties of the oligodendrocytes Lee and colleagues have developed an in vitro myelination assay where the oligodendrocytes were cultures with synthetic fibers of various calibers. The authors describe an axon-independent myelination presenting similar molecular and morphological features to in vivo myelination indicating that until certain extend the myelination may occur in the absence of axonal signaling (Lee et al., 2012).

\subsection{Aims of the study}

The aim of this study was to obtain an in-depth knowledge of the morphology of the growing myelin sheath and to shed some light on key mechanisms and features of the myelination process to understand the modalities of the myelin wrapping in the mammalian CNS. We used advanced microscopy techniques, genetics and molecular tools to investigate the formation of the myelin sheath, to identify the growth front of the myelin and to characterize new features associated to the myelin growth.

Based on our observations we propose a new model of myelination in the CNS, where the advancing inner tongue is winding around the axon in the center of the segment leading to the radial growth of the myelin sheath. Concurrently to this first growth motion the successive layers would extend laterally toward the lateral edges of the internode until completion of a uniformly thick segment. 


\section{Materials and Methods}

\subsection{Materials}

\subsubsection{Equipment for electron microscopy}

To process samples by high pressure freezing, the HPM 100 (Leica) was used followed by a freeze substitution carried out in an AFS2 unit (Leica)

The samples embedded in EPON or cryo-fixed for cryo immune electron microscopy were cut using respectively a Leica Reichert Ultracuts microtome and a Cryo microtome Leica EMUC6 (Leica).

The imaging of the samples was done on an EM LEO 912 (Zeiss) for the transmission electron microscope and on a Helios Nanolab 650 dual beam (FEI) for the FIBSEM.

\subsubsection{Equipment for light microscopy}

Attained primary oligodendrocyte cell cultures were imaged by a Zeiss LSM 510 or a Leica DMI 6000. The live imaging on Zebrafish animals was carried out on a Zeiss LSM 710 with live imaging setup.

\subsubsection{Chemicals}

2.1.3.1 For electron microscopy

\begin{tabular}{|l|l|}
\hline Name & Origin \\
\hline Led citrate 8473 & Merck \\
\hline Uranyl acetate & Merck \\
\hline Paraformaldehyde & Serva \\
\hline Glutaraldehyde & Electron Microscopy Science \\
\hline OSO4 (osmium tetroxide) & Electron Microscopy Science \\
\hline Glycinether & Serva \\
\hline DDSA (2- Dodecenylsuccinic acid anhydride & Serva \\
\hline MNA (methylnadic anhydride) & Serva \\
\hline
\end{tabular}




\begin{tabular}{|l|l|}
\hline DMP30 & Electron Microscopy Science \\
\hline Tanic acid & Sigma \\
\hline Aceton glass distilled & Electron Microscopy Science \\
\hline Polyvinylpyrrolidone & Sigma \\
\hline
\end{tabular}

2.1.3.2 For cell culture

\begin{tabular}{|l|l|}
\hline Name & Origin \\
\hline B27 supplement & Gibco/Invitrogen \\
\hline DMEM for Primary cell culture & Gibco/Invitrogen \\
\hline DMEM for cell line culture & PAA \\
\hline Fetal Calf Serum (FCS) & PAA \\
\hline GlutaMAX ${ }^{\text {TM}}$-I supplement & Gibco/Invitrogen \\
\hline Horse Serum & PAA \\
\hline Trypsin/EDTA & Lonza GmbH \\
\hline OptiMEM-I Media & Invitrogen \\
\hline PBS & PAA \\
\hline Poly-L-Lysine (PLL) & Sigma \\
\hline Penicillin/Streptomycin (Pen/Strep) & Gibco/Invitrogen \\
\hline Lipofectamine2000 & Invitrogen \\
\hline
\end{tabular}

2.1.3.3 Inhibitors for in vitro studies

\begin{tabular}{|l|l|l|}
\hline Name & concentration & Origin \\
\hline Genistein & $25 \mu \mathrm{M}$ & Sigma \\
\hline Wortmannin & $200 \mathrm{nM}$ & Sigma \\
\hline Ly294002 & $1 \mu \mathrm{M}$ & Calbiochem \\
\hline Akt $1 / 2$ kinase inhibitor & $500 \mathrm{nM}$ & Sigma \\
\hline VO-OH pic & $200 \mathrm{nM}$ & BioVision \\
\hline Rapamycin & $10 \mathrm{nM}$ & Sigma \\
\hline
\end{tabular}




\subsubsection{Plasmid}

In this study we transfected oligodendrocyte cell culture with the following plasmid 16643: PI3KCA-WT and 21218: GFP-C1-AKT-PH (addgene) using the protocol described in 2.2.1.2

\subsubsection{Animal lines}

The live imaging was performed on the following transgenic zebrafish lines: $\operatorname{Tg}(n k x 2.2 a: m E G F P)$ and $\operatorname{Tg}(m b p: E G F P-C A A X)$.

The PTEN cre/+ flox/flox Pten and CNP mouse mutants used in this studies were previously described in Goebbles et al., 2010 and Lappe-Siefke et al., 2003 and were compared with control littermate animals. The shiverer mice lacking MBP were maintained on a C57BI6 background and were compared with control littermate animals.

\subsubsection{Antibodies (primary and secondary)}

\subsubsection{Primary}

\begin{tabular}{|l|l|l|l|l|}
\hline Name & Isotype & Light microscopy & Electron microscopy & Origin \\
\hline MBP & Rb polyclonal & $1: 200$ & $1: 100$ & Dakocytomation \\
\hline CNP & Mouse IgM & $1: 200$ & $1: 50$ & Sigma-Aldrich \\
\hline P-Akt 473 & Rb polyclonal & $1: 100$ & & Cell signaling \\
\hline PIP3 & Mouse IgM & $1: 100$ & $1: 25$ & $\begin{array}{l}\text { Echelon } \\
\text { Bioscience }\end{array}$ \\
\hline MAG & Mouse IgM & $1: 100$ & & Millipore \\
\hline VSV-G & Rb polyclonal & $1: 2000$ & $1: 1000$ & \\
\hline PAG & & & $1: 50$ & CMC Utrecht \\
\hline
\end{tabular}

\subsubsection{Secondary}

\begin{tabular}{|l|l|l|l|}
\hline Name & Isotype & Light microscopy & Origin \\
\hline Alexa 488 & goat IgM & $1: 400$ & Invitrogen \\
\hline Alexa 555 & goat IgM & $1: 400$ & Invitrogen \\
\hline Alexa 647 & goat IgM & $1: 400$ & Invitrogen \\
\hline Phaloidinrodamine & & $1: 200$ & Invitrogen \\
\hline
\end{tabular}




\subsubsection{Buffer list}

10X phosphate buffered saline (PBS)

$80 \mathrm{~g} \mathrm{NaCl}$

$2 \mathrm{~g} \mathrm{KCl}$

14.4 g Na2HPO4 (or 18.05 g Na2HPO4_ 2H2O)

$2.4 \mathrm{~g} \mathrm{KH} 2 \mathrm{PO} 4$

Add $\mathrm{dH} 2 \mathrm{O}$ to $1 \mathrm{~L}$ and adjust $\mathrm{pH}$ to $7.05-7.11$

\section{Super SATO medium}

$2 \%$ B27-supplement

$1 \%$ Horse serum

$110 \mu \mathrm{g} / \mathrm{mL}$ pyruvate

500 pM tri-iodo-thyronine

520 nM L-thyroxine

1X Pen/Strep

1X GlutaMAX ${ }^{\text {TM }}$

in DMEM (Gibco/Invitrogen) with high glucose and without glutamine

\section{Steinberg buffer}

$58 \mathrm{mMNacl}$

$0,67 \mathrm{mM} \mathrm{Kcl}$

$0,44 \mathrm{mM} \mathrm{Ca}(\mathrm{NO} 3) 2$

1,3mM MgSO4

4,6mMTrizma base

$\mathrm{pH} \mathrm{7,8-8,0}$ 


\section{Mowiol}

2.4 g Mowiol

6 g Glycerol

$6 \mathrm{~mL} \mathrm{H} 2 \mathrm{O}$

$12 \mathrm{~mL} 0.2 \mathrm{M}$ Tris/HCl

$\mathrm{pH} 8.5$

2X Karlsson and Schultz phosphate buffer (1965)

0,09g NaH2PO4 x H2O

0,775g Na2PO4 x H2O

$0,25 \mathrm{~g} \mathrm{NaCl}$

$25 \mathrm{ml} \mathrm{H} 2 \mathrm{O}$

$\mathrm{pH} \mathrm{7,4}$

\subsubsection{Software}

\begin{tabular}{|l|l|l|}
\hline Software & Application & Source/Manufacturer \\
\hline Adobe Photoshop CS5.1 & Image processing & Adobe Systems, Inc \\
\hline ImageJ/ FIJI & Image processing and analysis & http://rsbweb.nih.gov/ij/ \\
\hline Leica Software & images acquisition & $\begin{array}{l}\text { Leica Microsystems, } \\
\text { Mannheim, Germany }\end{array}$ \\
\hline Zen Software & Confocal images acquisition & Zeiss, Inc. \\
\hline Imod & Segmentation tool/3D modeling & Boulder Laboratory \\
\hline CorIDRAW X5 & Drawing software & Corel corporation \\
\hline Slice and view & Dual beam acquisition software & FEI Company \\
\hline Amira & Alignment software & FEI Company \\
\hline Adobe Illustrator CS5.1 & Figure design & Adobe Systems, Inc. \\
\hline EndNote & Bibliography manager & Thomas Reuters \\
\hline
\end{tabular}




\subsection{METHODS}

\subsubsection{Cell culture techniques}

\subsubsection{Primary Oligodendrocyte cultures}

The primary oligodendrocyte cell cultures were prepared from the brain of postnatal day 0-1 mice as described previously (Trajkovic et al., 2006). In short, after removing the meninges, the brains were digested with $0.25 \%$ trypsin, and cultured in Eagle's basal medium with $10 \%$ horse serum on poly-L-lysine (PLL)-coated flasks at $37{ }^{\circ} \mathrm{C}$. The oligodendrocytes were then harvested from 8-10 day old mixed glia culture using mechanical shaking and further cultured on PLLcoated 11-13 mm glass coverslips in Super SATO medium (see materials section) (fig 2.1).
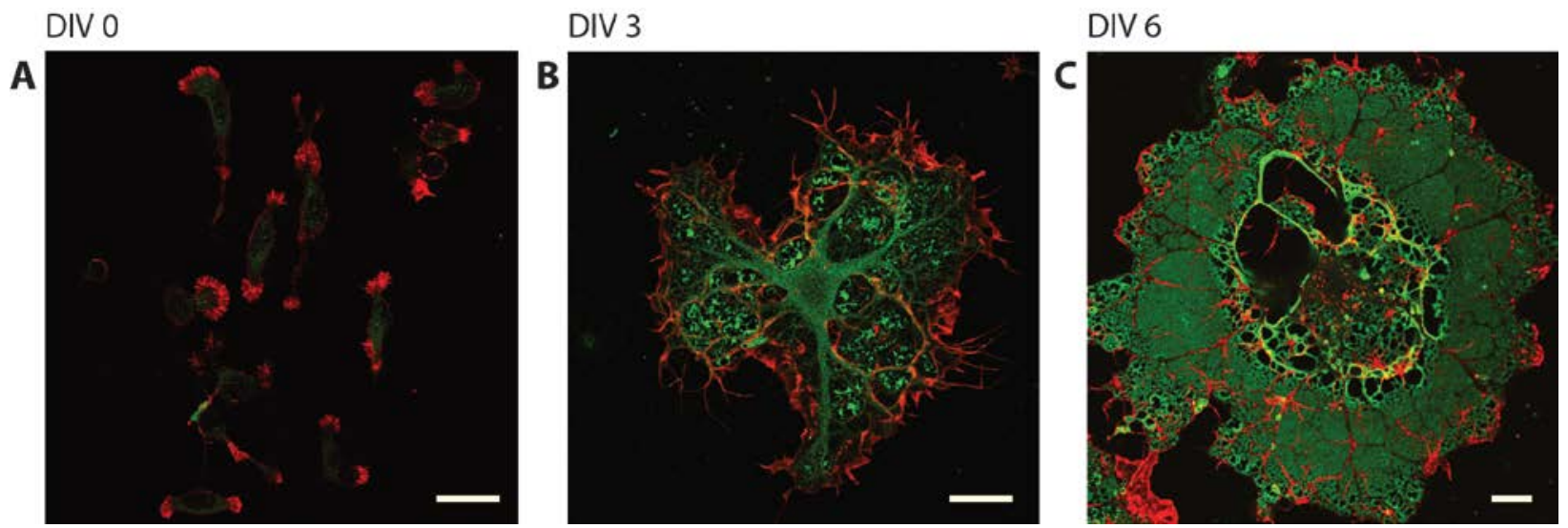

Figure 2.1: In vitro model of myelination: the primary oligodendrocyte cell culture

A-C) Evolution of the morphology of the cultured oligodendrocyte in culture over 6 days. Filamentous actin is labeled in red and MBP in green. Scale bar $20 \mu \mathrm{m}$.

\subsubsection{Primary Oligodendrocyte cells transfection}

Transfection of primary cultures was done using Lipofectamine 2000. Oligodendrocyte cells were cultured on 11-13 mm glass coverslips in 24 well plates for 2-3 days after shaking. The following reaction was prepared for each coverslip: $1.6 \mu \mathrm{g}$ of plasmid DNA was mixed with $100 \mu \mathrm{L}$ of OptiMEM-I. Separately, $4 \mu \mathrm{L}$ of Lipofectamine2000 were mixed with $100 \mu \mathrm{L}$ OptiMEM. 
After 5 min of incubation, both solutions were mixed and incubated for $20 \mathrm{~min}$ at RT. The 200 $\mu \mathrm{L}$ of solution were then added to the cells and left for 10 to 16 hours before analysis.

\subsubsection{Chemical treatment of the Primary oligodendrocyte cultures}

The chemical treatments on the oligodendrocyte cell culture were performed as followed. The cells were incubated for 2-3 days after shaking in Super SATO medium. The chemicals were diluted directly in the Super SATO medium to reach the appropriate concentration (see Materials for details). The cells were further incubated for 12-24 hours with the various chemicals and then fixed with 4\% PFA and $0.25 \%$ GA before immunolabeling.

\subsubsection{Infection of the Primary oligodendrocyte cultures by vesicular stomatitis virus (VSV)}

The handling, injection, incubation and fixation of the oligodendrocyte cell culture were done in a S2 facility with suitable equipment. The culture medium of the oligodendrocytes was extracted and preserved at $37^{\circ} \mathrm{C}$ during the VSV infection procedure. The cell cultures were inoculated by Wild type vesicular stomatitis virus particles $\left(1 \times 10^{11} \mathrm{pfu} / \mathrm{ml}\right)$ at a dilution of 1 in 20000 in fresh Super SATO medium for 15 minutes. After incubation the virus particles that did not penetrate the cells were removed by washing with fresh culture medium. The cells were then further incubated for 2 to 6 hours in their original medium before fixation and immunofluorescence staining.

\subsubsection{Electrical stimulation of Primary oligodendrocyte cultures}

15,000 cells from primary oligodendrocyte cell cultures were plated on a microslide setup from Ibidi (Ibidi) and grown for 3 days as described in 222 The electrical stimulation of the cell culture was performed by a continuous electrical current of 1volt/cm from a 30V DC power supply for 2 to 4 hours. The current was transmitter to the cell cultures using silver chlorite electrodes (RC3 electrodes) in conventional Steinberg solution connected to the setup by agar bridges as described in (Zhao et al., 2006) (fig 2.2). The cells were then fixed and further processed for immunofluorescence staining. 


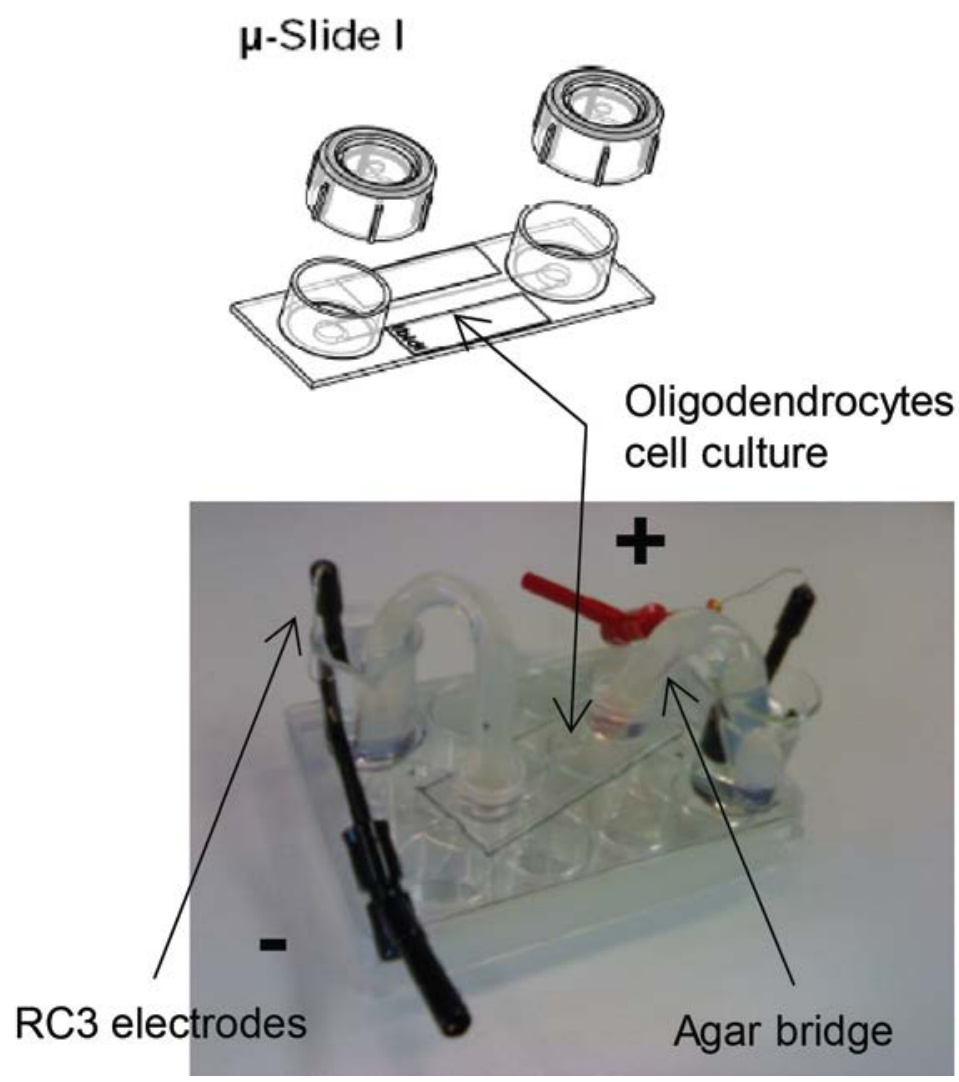

Figure 2.2: Setup for cultured oligodendrocyte electrical stimulation

Setup used to apply an electrical current to the cultured oligodendrocytes. The setup was constructed based on the work of Zhao et al., 2006.

\subsubsection{In vivo techniques}

2.2.2.1 Live imaging and electron microscopy of myelination in Zebrafish

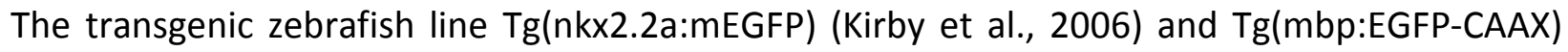
(Czopka and Lyons) were used for the live imaging of myelination events. Zebrafish embryos (3 days post fertilisation) were embedded in $1.5 \%$ low melting point agarose in embryo medium with Tricaine and imaged for several hours on a Zeiss LSM 710 with a heated stage.

The Zebrafish embryos used for electron microscopy were high pressure frozen at a similar age than the animals imaged with the light microscopy studies and at a similar area of the spinal cord was assessed.

All animals were maintained in accordance with UK Home Office guidelines. 


\subsubsection{Mice handling and mutants}

Animals were sacrificed accordingly to FELASA guidelines by cervical dislocation or Avertine injection followed by transcardial perfusion. The animals were sacrificed at various time points to assess the myelination during development depending on the tissues. Concerning the optic nerve investigations, the nerves were prepared from animals 10, 14, 21-23 and 60 days after birth. For the spinal cord study the samples were prepared from thoracic segment of P4 and P60 animals. The corpus callosum samples were obtained from P21 and P60 animals. The generation of PTEN cre/+ flox/floxPten and CNP mutant mice has been described previously (Goebbels et al.; Lappe-Siefke et al., 2003). The shiverer mice lacking MBP were maintained on a C57BI6 background.

\subsubsection{Sample preparation of mouse central nervous system tissues}

Various mouse nervous tissues samples were prepared for various experiments and thus the preparation of these samples had to be adapted. The optic nerves used on the morphological studies were extracted within 3 minutes after cervical dislocation and directly high pressure frozen by the HPM 100 (fig 2.3). The spinal cord samples were obtained from thoracic sections of perfused animals (see section 2.2.3.1) and further proceeded for cryo-immuno electron microscopy. The corpus callosum samples were extracted from perfused animals' brain at the injection side and further proceeded for cryo-immuno electron microscopy.

\subsubsection{Intracerebral injection of VSV in Corpus callosum}

For intracerebral (i.c.) infection, wild type mice were anesthetized using isoflurane inhalation anesthesia. The VSV inoculation $(10 \mu \mathrm{l})$ was performed using a $28 \mathrm{G}$ insulin syringe over a period of $3 \mathrm{~min}$ in balanced salt solution with $0.3 \%$ of monostral blue. The latter was added for better visualization of the injection side for histological processing. Following the i.c. injection the animals were euthanized either 3 or 6 hours later by intraperitoneal injection of pentobarbital and transcardially perfused with 4\% PFA. Subsequently, brains were dissected, and small pieces

of cortex with corpus callosum were postfixed with PFA for $4 \mathrm{hr}$ and further processed for immunoelectron microscopy. 
A
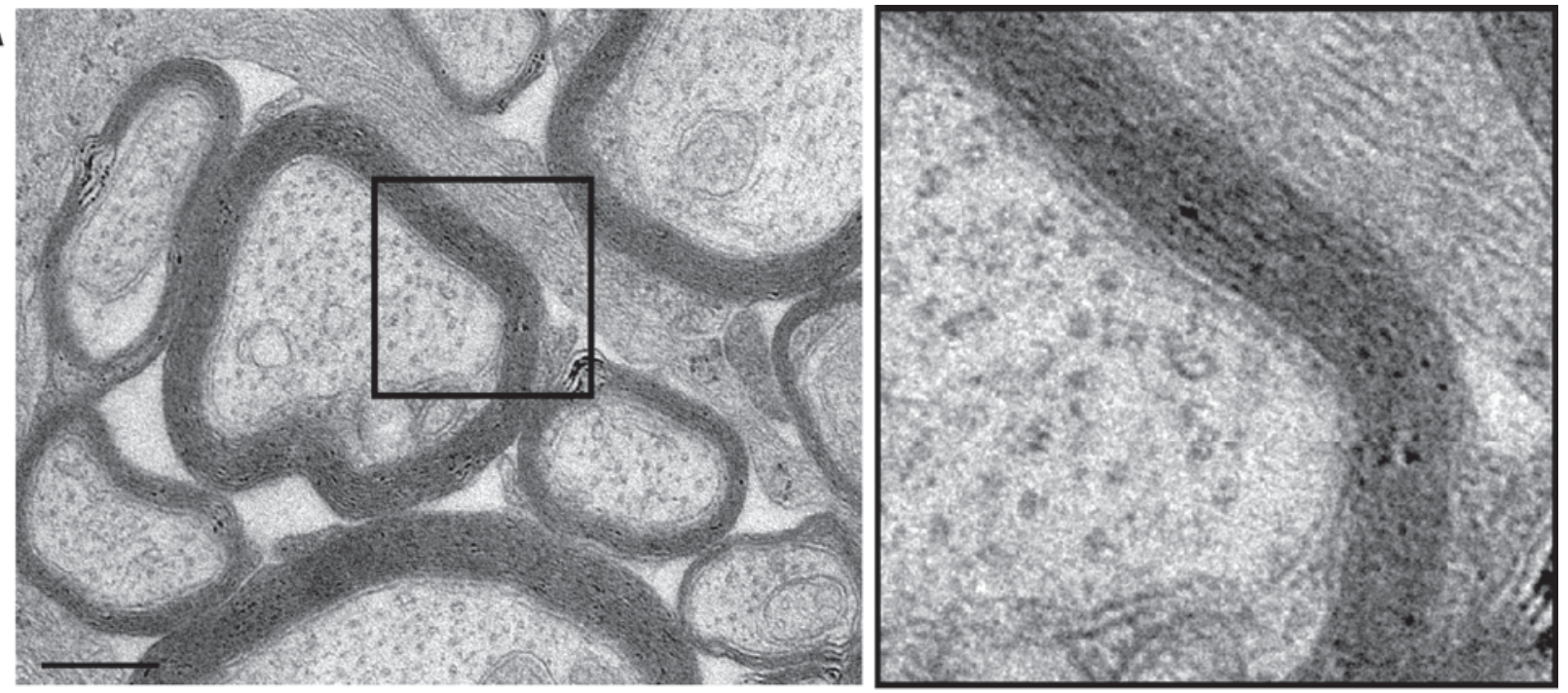

B
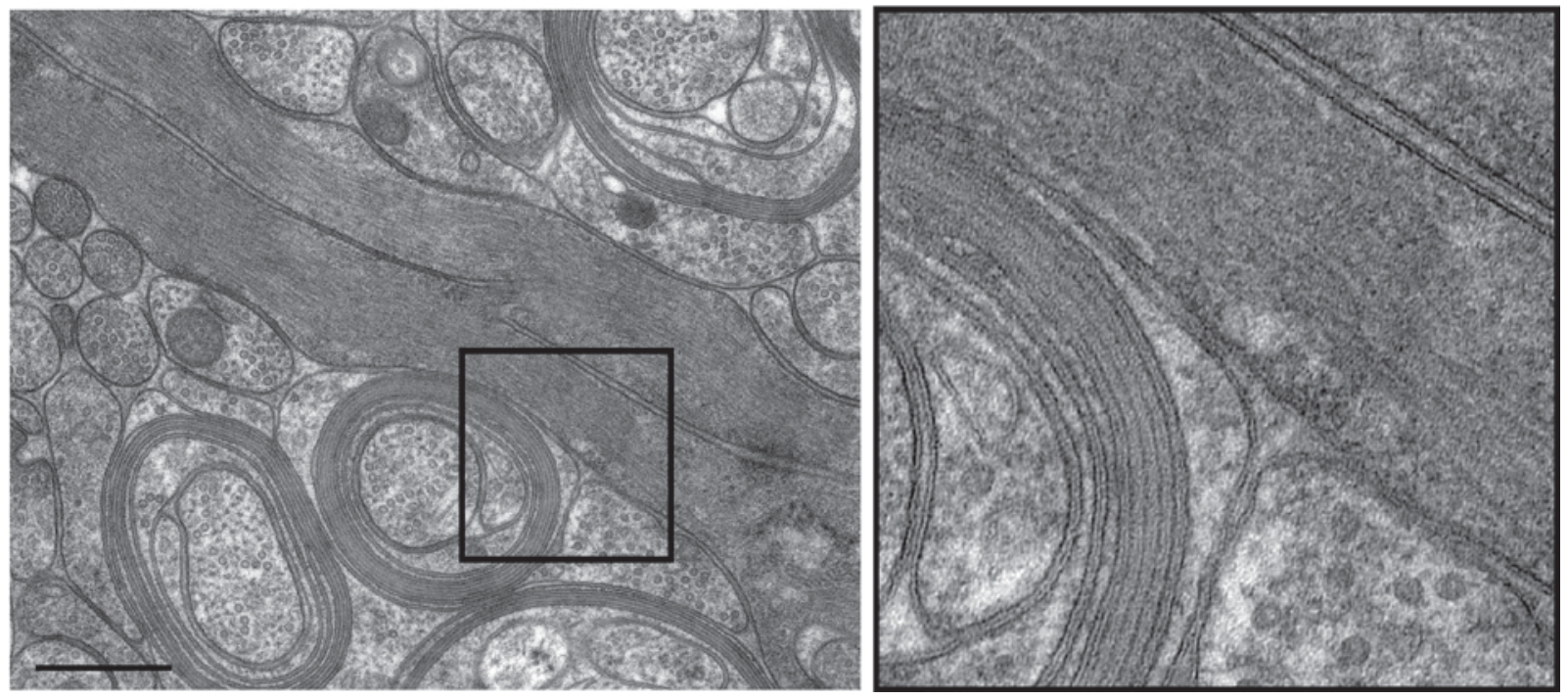

Figure 2.3: Myelin morphology preservation by high pressure freezing

A) Electron micrograph obtained from an optic nerve after conventional chemical fixation for 4 hours. B) Optic nerve after high pressure freezing on freshly extracted optic nerves followed by freeze substitution. Scale bar $500 \mathrm{~nm}$.

\subsubsection{Fixation and staining}

\subsubsection{Chemical fixation and perfusion}

The chemical fixation of the oligodendrocyte cell culture and the mouse tissues destined to be process for immunofluorescence or cryo-immuno electron microscopy were realized by a combination of Paraformaldehyde (PFA) and Glutaraldehyde (GA). Fresh PFA and GA stock 
solutions were used for every fixation with a final concentration of PFA and GA equal to 4 percent and 0,25 percent respectively in Karlsson and Schultz phosphate buffer. The oligodendrocyte cell cultures were fixed for 15 minutes at RT before further processing. The optic nerves were freshly extracted and immerged in fixative for 2 hours at RT before further processing.

Concerning the perfusion procedure, the animals were first sedated with Avertine injected intraperitonealy, flushed from their blood by $5 \mathrm{ml}$ of HBSS and perfused transcardially for 20 minutes with PFA/GA. The brain and the spinal cord were then extracted and further postfixed in PFA/GA for 2 hours and in PFA only over night at $4^{\circ} \mathrm{C}$ before further processing.

\subsubsection{High pressure freezing fixation}

Mice were killed by cervical dislocation and the optic nerves were extracted within 3 minutes post dislocation. The Optic nerves were loaded on a freezer hats (Leica) using Polyvinylpyrrolidone as a filler and then high pressure frozen within 5 minutes post cervical dislocation. The samples were further processed by freeze substitution and EPON-embedding protocol described in Mobius et al 2010. In short the samples were incubated in Tannic acid for 98 hours at $-90^{\circ} \mathrm{C}$, washed, incubated in $2 \%$ OsO4/ $0.25 \%$ Uranyl acetate for 40 hours going from $-90^{\circ} \mathrm{C}$ to $4^{\circ} \mathrm{C}$, washed and then embedded in EPON.

\subsubsection{Immunocytochemistry}

The fixed cells were first permeabilized with $0.1 \%$ Triton X-100 in PBS for 1-2 min then the blocking solution (2\% BSA, $0.2 \%$ Fish gelatine, and $2 \%$ FCS in PBS) was added for 30 min at RT. The primary antibodies diluted in blocking solution were added for $1 \mathrm{~h}$ at RT (see section 2.1.6.1). After three successive washing steps with PBS the cells were incubated with the secondary antibodies for 45 minutes at RT (see section 2.1.6.2 for the dulutions). Finally, the coverslips were washed with PBS and mounted onto a glass slide with a drop of Mowiol solution before imaging. 


\subsubsection{Immuno-electron microscopy staining}

The samples used for immuno-electron microscopy were fixed as described in 2.2.3.1 with a mixture of PFA and GA to optimize the ultrastructure preservation and allow efficient epitope recognition by the antibodies. The labeling was performed on $50 \mathrm{~nm}$ cryo sections according to the Tokuyasu method as described previously (Werner et al, 2007). In brief, after fixation the samples were embedded in $10 \%$ gelatin (Twee Torens), infiltrated in 2,3 M sucrose over-night at $4^{\circ} \mathrm{C}$, mounted on an aluminum pin and frozen in liquid nitrogen. The samples were then cut, $50 \mathrm{~nm}$ thick, at $-110^{\circ} \mathrm{C}$ for the optic nerves and corpus callosum and cut, $300 \mathrm{~nm}$ thick, at $-80^{\circ} \mathrm{C}$ for the spinal cord samples and then picked up in a mix of sucrose/methyl cellulose. The samples were then proceeded for labeling as described in Peters and Pierson (2008). The pickup solution was removed by PBS washes, the aldehydes quenched by $0.1 \%$ glycine in PBS, the sample blocked by 1\%BSA in PBS, then the first antibodies were applied for 1 hours at RT and the samples were washes in 0,1\%BSA in PBS before second antibody was applied for 45 minutes at RT (if the first antibody was not rabbit). After the second antibody the samples were washed and incubated with PAG (protein A gold) (10nm gold) for 30 minutes then further washed, fixed in $1 \% \mathrm{GA}$ and contrasted by neutral uranylacetate for 5 minutes at RT and Methylcellulose/uraylacetate (9/1) for 5 minutes on ice before drying.

\subsubsection{Imaging}

\subsubsection{Transmission electron microscopy}

The ultrathin cross and longitudinal sections of high pressure frozen optic nerves and the samples for immuno-electron microscopy were imaged by transmission electron microscopy on a LEO 912 set on $80 \mathrm{kV}$. The samples were imaged at 6300-12000X by a 2K CCD camera (TRS) by single frame or by multiple image acquisition (MIA) allowing a larger field of view used for quantification purposes. Three to five animals were used for each TEM analysis for every time point and five to ten areas of $150{\mu \mathrm{m}^{2}}^{2}$ were imaged allowing the assessment and quantification of 100 to 300 axons per animal. The myelin quantifications were done on myelinated axons presenting at least 4 compacted or uncompacted wraps. 


\subsubsection{Serial focus ion beam milling / scanning electron microscopy}

The samples imaged by the Helios Nanolab 650 (FEI, The Netherlands) were high pressure frozen P10 optic nerves processed as described in Moebius et al in 2010. The optic nerves in EPON blocks were imaged on a Helios Nanolab 650 dual beam (FEI) with a $2 \mathrm{kV}$ electron beam using the back scattered electron mode (immersion lens). The milling of the block face was achieved by a gallium beam that would remove $50 \mathrm{~nm}$ of sample at each cycle. The block face was sequentially imaged using the slice and view software with a dwell time of $3 \mu \mathrm{s}$.

The volumes of the acquisition were covering $16 \times 13,8 \times 40-55 \mu \mathrm{m}^{3}$ with a $x / y$ pixel size of 4-6 $\mathrm{nm}$ and were recorded for 4 to 7 days for each sample. The alignment and segmentation of the volumes were obtained using the Amira and IMOD (Kremer et al 1996) software allowing the 3D reconstruction of myelination patterns.

\subsubsection{Light microscopy imaging}

Oligodendrocyte cell cultures were stained as described in (Trajkovic et al., 2006) and imaged with a LSM 510 (Zeiss) or a DMI 6000 (Leica) using 40X or 63x objectives. Each experiment involving oligodendrocytes cell culture was performed on 3 independent primary cell cultures based on at least 30 cells per coverslip.

The Zebrafish images were taken from lateral views of the spinal cord of immobilized animals, with anterior part on the left and dorsal part upwards. The time-lapse imaging was carried out on a Zeiss LSM 710 (Zeiss) with a heated stage. Confocal images were obtained with $\mathrm{x}, \mathrm{y}$ resolution and with z-step intervals optimised for subsequent image deconvolution.

\subsubsection{Image processing and analysis}

2.2.4.4.1 Transmission electron microscopy image analysis

The cross section electron micrographs of high pressure frozen samples were used to count the amount of wraps, the cytoplasmic channels, inner and outer mesaxon areas and the areas covered by vesicles on 20 randomly selected axons per MIA $\left(150 \mu \mathrm{m}^{2}\right)$. The proportion of myelin sheath presenting cytoplasmic channels and outfoldings was quantified based on the entire axon population (with a myelin sheath thicker than 4 warps) from the acquired MIA. The frequency of myelin outfoldings at P10 was determined as follows: we estimated that an 
outfolding covers approximately $10 \%$ of the internodal length (the outfoldings have a length of $\sim 10 \mu \mathrm{m}$ and the internode of $\sim 100 \mu \mathrm{m})$. Considering that $6.5 \%$ of the myelinated axons have outfoldings in a cross section, we estimated that the frequency of myelin outfoldings is $~ 65 \%$ (6.5×10) per myelin segment in the optic nerve at P10.

Longitudinal electron micrographs were used to assess the positioning and morphology of successive myelin layers along the forming myelin sheath as well as the node formation. The quantifications were performed on myelin segment at least $10 \mu \mathrm{m}$ long for 20 segments per animal.

2.2.4.4.2 Cultured oligodendrocyte cell rim quantification and surface VSV-G distribution

In order to measure the rim size of the oligodendrocytes during development as well as upon treatments, the F-actin and CNP positive areas were measured at 4 opposite quadrants of the cells. The average value was calculated for each cell and 30 cells per culture were assessed.

The distribution of the VSV-G surface labeling was obtained by measuring the signal within a region of interest of $40 \times 20 \mu \mathrm{m}$. The signal was plotted on a 2D graph representing the signal from the rim of the cell and the signal from a $40 \mu \mathrm{m}$ distance from the cell surface toward the nucleus. The average distribution was calculated from 3 independent cultures for 30 cells per culture.

\subsection{Zebrafish quantification}

The fluorescence intensity measurement for both transgenic lines was achieved by measuring the fluorescence intensity along the length of the sheath on a slice of the z-stack which showed an accurate representation. Then a region of interest around the sheath was selected and the intensity was plotted. The values were expressed as percentage of the maximal brightness along the length of the sheath. 


\subsection{D segmentations of the FIBSEM data}

The three dimensional reconstruction of myelination pattern based on the block face imaging of the FIBSEM data was achieved by segmentation of the inner mesaxon membrane (orange traces), the outer mesaxon and the oligodendrocyte cell body membrane (purple traces), the compact myelin (cyan traces), the cytoplasmic channels (red traces) and vesicular pattern (blue traces) from all the section of the volumes.

\subsection{Immuno-electron microscopy labeling}

The MBP labeling quantification of the compacted and non-compacted areas of the growing sheath was performed on P10 optic nerve on myelin sheath presenting non-compacted and compacted myelin wraps. The amount of MBP labeling was reported to the membrane length in both the compacted and non-compacted wraps giving the labeling per $\mu \mathrm{m}$ of membrane.

The quantification of the VSV-G labeling was performed in the corpus callosum on 100 axons wrapped by infected myelin sheath in 3 littermate animals for the 2 following conditions: 3 and 6 hours after VSV injection at P21 and P60. The VSV-G labeling was measures in the compacted myelin sheath, the inner mesaxon and the outer mesaxon.

\subsubsection{Statistics}

All quantifications were represented as the mean value +/- standard error of the mean. Significance was determined by Student t-test. 


\section{Results}

\subsection{Morphology of the growing myelin sheath in vivo.}

The oligodendrocytes in CNS show one of the most drastic morphological changes and final complex architecture achieved by a single cell in our body. Indeed the oligodendrocyte is capable to generate up to 50 myelin segments of tightly packed membrane layers reaching a defined myelin thickness in a time span of days.

The morphological investigations of the myelin sheath and its formation have been the subject of intense research. However, due to technical limitations it has not yet been possible to experimentally support any of the proposed models.

In this study we made use of an integrative approach employing advanced imaging techniques, such as high resolution volume microscopy using serial focus ion beam SEM slices (FIB SEM), high pressure freezing TEM and in vivo live imaging in Zebrafish, to investigate the morphology of the growing myelin sheath.

\subsubsection{Three dimensional reconstructions of growing myelin sheaths by FIB-SEM}

\subsubsection{Technical approach to image a large volume at nanometer resolution range}

Over the last 60 years the morphological studies on CNS and PNS myelin were carried out at nanometer scale with the transmission electron microscopy (TEM) and at the micrometer range with light microscopy. Electron microscopy allows the visualization of tissues with a resolution sufficient to identify individual myelin layers and most of the subcellular structures, whereas the light microscopy allows a larger field of view, visualization of fluorescent proteins, immunofluorescence staining and live imaging.

However, these two imaging techniques have specific limitations. Due to the very small sample size assessable by TEM (few $10^{\text {th }}$ of $\mu \mathrm{m}$ in $\mathrm{x} / \mathrm{y}$ and $50 \mathrm{~nm}$ in $\mathrm{z}$ ), the spatial organization of an entire CNS myelin segment during development (around 100 $\mu \mathrm{m}$ of length, 1-2 $\mu \mathrm{m}$ of diameter in the optic nerve) appeared to be complex to obtain. The light microscopy, on the other hand, would allow the visualization of an entire myelin segment and even the localization of specific 
proteins, but due to the diffraction limit of the light, the resolution is insufficient to resolve the structure of the myelin sheath.

It became clear that to understand the spatial organization of the growing myelin sheath we would need another imaging technique. We used a recent technical development of the scanning electron microscopy applied to live sciences - the Focus ion beam milling coupled to a scanning electron microscope (FIB-SEM) - to obtain a large volume of data with high resolution. The principle of this microscope is to couple one ion beam that will remove a thin layer of the sample and one electron beam that will image the surface of the sample (fig 3.1). These processes can be automatically repeated theoretically ad libitum by recognition of fiducials on the sample allowing a serial view of the sample over a large area in three dimensions.

We used the FIBSEM at the FEI Company (FEI Company, Eindhoven, The Netherlands) on high pressure frozen P10 optic nerves. We could, over 4 to 8 days of uninterrupted recordings, acquire several volumes of data as big a $16 \mu \mathrm{m}$ in $X * 14 \mu \mathrm{m}$ in $Y * 40-55 \mu \mathrm{mZ}$ in $Z$ with the $X, Y$ resolution around $4-6 \mathrm{~nm}$ and a distance between slices of $50 \mathrm{~nm}$ ( $\mathrm{z}$ resolution) (fig 3.2, video 01). The imaging of the samples was possible using the back-scattered detector of the electron beam that would image the structures stained with heavy metal within the few first nanometers of the sample (fig 3.2). The resolution of the FIB-SEM was sufficient to assess the morphology of the non-compacted area of the myelin, the axons and their content, the vesicles, mitochondria and even elements of the cytoskeleton such as the microtubules (fig 3.2).

Thus the FIB-SEM on high pressure frozen samples appeared to be the most suitable imaging technique to investigate the morphology of the developing myelin sheath.

\subsubsection{Three dimensional visualization of myelination events at high resolution}

Based on the volumes acquired with the FIB-SEM we could generate three dimensional reconstructions of 45 axons and their myelin sheath during the wrapping process (fig 3.3). These 3D visualizations were composed of the reconstructions of the axon, the outer tongue 
and the inner tongue. Indeed, the relative positioning of these 2 cytoplasmic rich areas gave us the possibility to understand the organization of the myelin layers along the developing sheath.

A

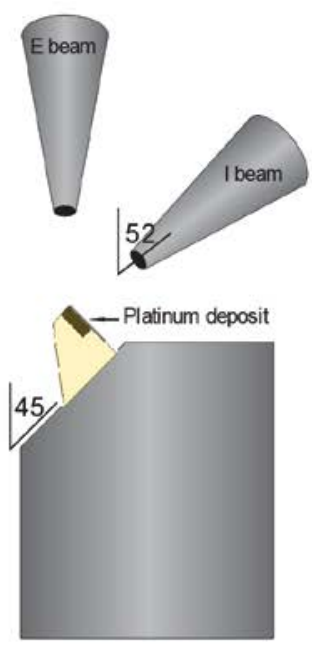

B
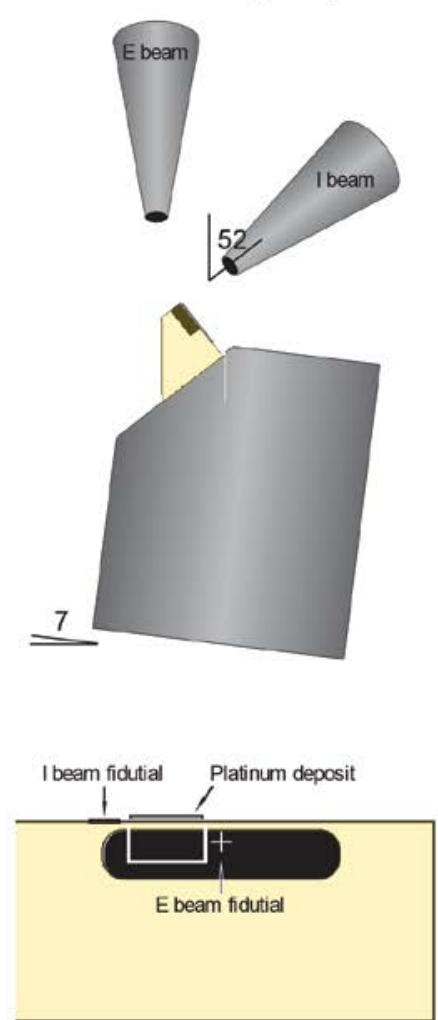

C

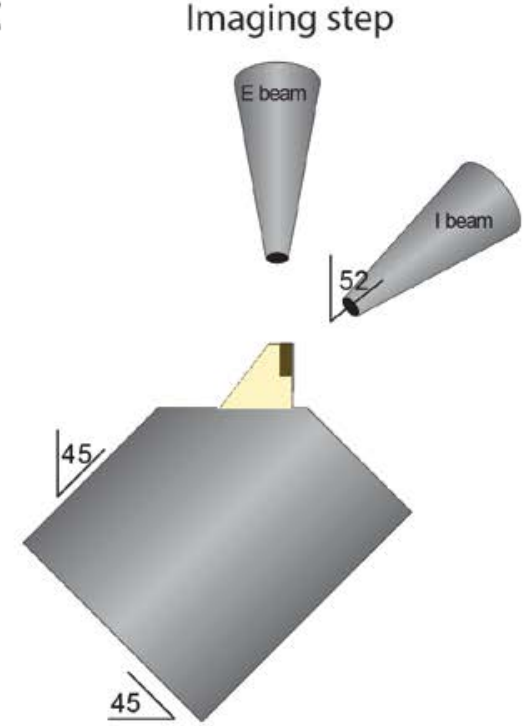

Fidutials on resin block

\section{Figure 3.1: Sequential sample milling and imaging position during block face imaging}

A) Initial positioning of the stage in the focus ion beam scanning electron microscope (FIBSEM) with the sample mounted on a $45^{\circ}$ aluminum pin. B) The sample is inclinated by 7 degrees to align the ion beam with the surface of the sample. The surface of the sample is then removed by the ion beam. C) Next, the stage moves then by $45^{\circ}$ to align the surface of the sample with the electron beam and to further proceed with the imaging. D) Positioning of the fiducials deposited or milled within the sample allowing the automated repeat of the milling and imaging sequence.

Amongst the analyzed myelin sheath we show a representative segment (pointed in red in fig 3.3) from which we made a detailed segmentation of the axolemma (in green), inner tongue (in orange), compact myelin (in cyan), outer tongue and oligodendrocyte cell body (in purple) and other cytoplasmic rich areas within the myelin sheath (fig 3.4, video 02). The detailed 3D reconstruction allowed us to visualize the oligodendrocyte cell body and its connection to the growing myelin sheath by the oligodendrocytic process (purple) in direct continuity with the 
outer tongue towards the two ends of the myelin segment. We found that the outer tongue shows only a minor winding around the growing sheath and accordingly the outer layer of the myelin would spans almost the entire segment length (fig 3.5). In contrast, the inner tongue would wind far more often (approximately ten times more) around the axon than the outer tongue and thus would be responsible for the increase/decrease of the myelin thickness along the myelin segment (fig 3.5).
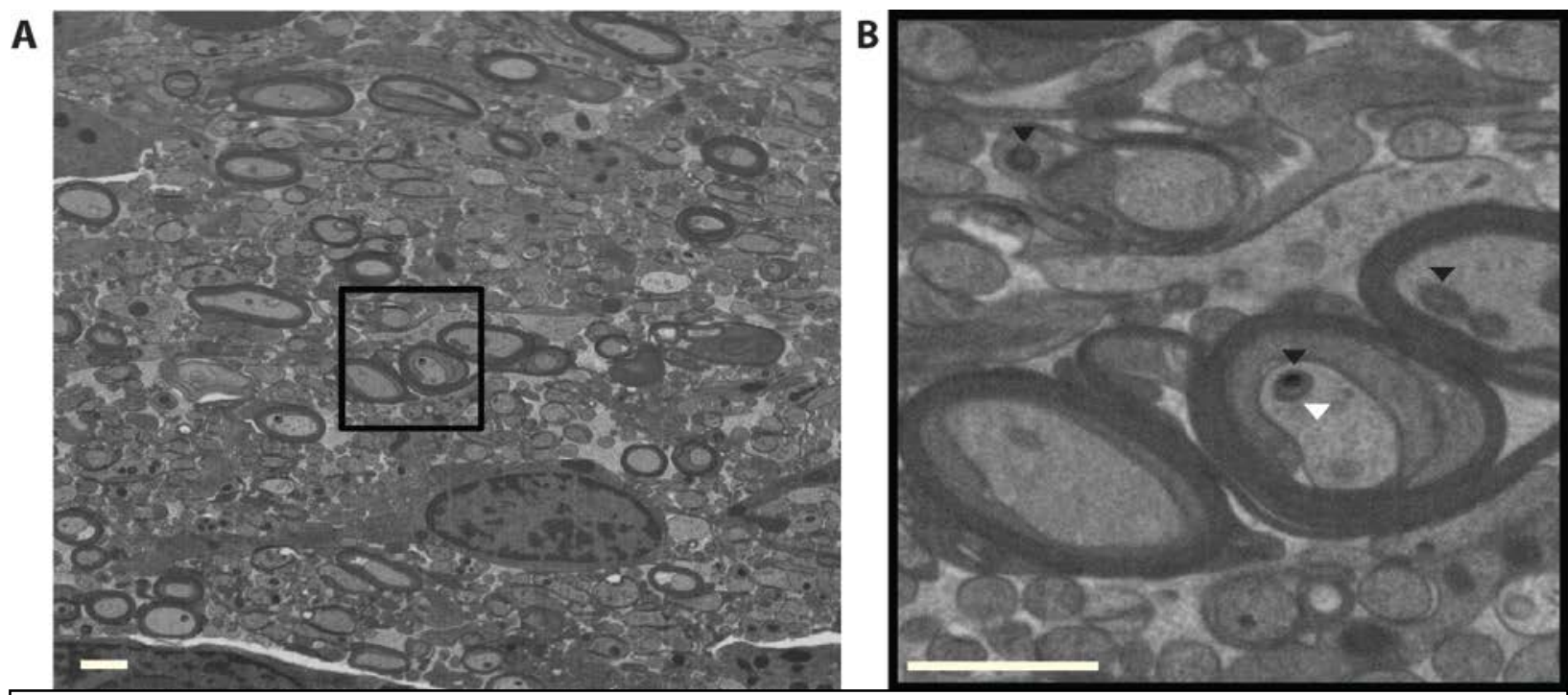

Figure 3.2: Resolution and cellular ultrastructure assessed by FIBSEM imaging

A) Selected slice of the volume of data acquired with the FIBSEM on P10 high pressure frozen optic nerve. The acquired area reaches $16 \mu \mathrm{m}$ in width and $14 \mu \mathrm{m}$ in height. B) Enlarged view on several myelinated axons where we could identify microtubules (white arrow heads), mitochondria (black arrow heads) vesicles (blue arrow heads) and clearly distinguish the compacted from the non-compacted areas in the myelin sheath. Scale

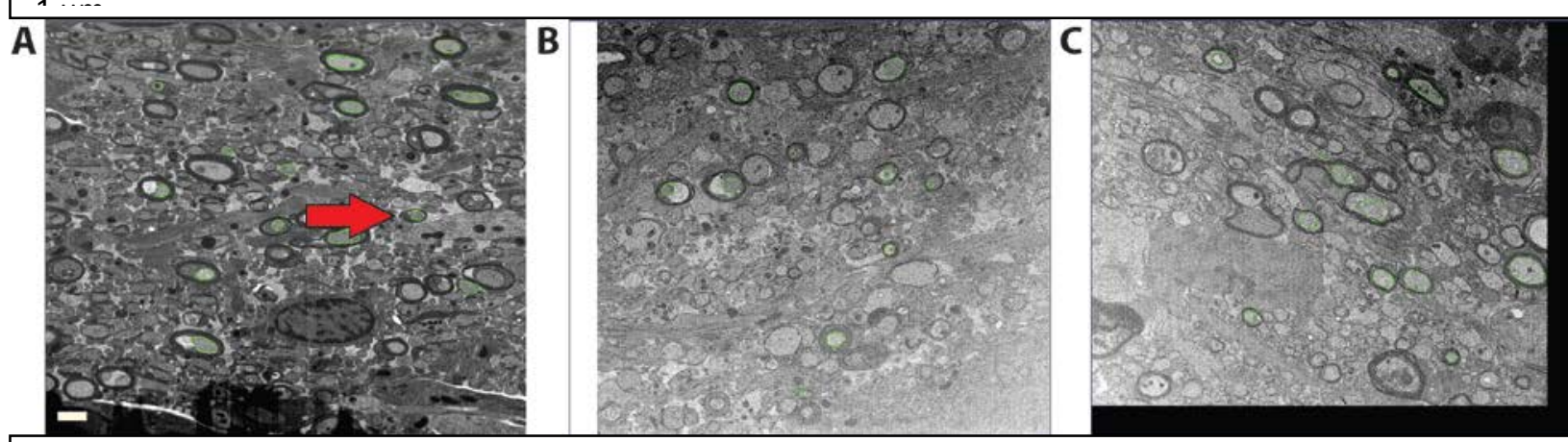

Figure 3.3: Three dimensional high resolution investigation of myelin biogenesis

A-C) Selected slices of the 3 volumes acquired from 3 different samples by FIBSEM. The myelinated axons that remained in the frame of the acquisition (labeled in green) were segmented and a model was generated allowing three dimensional investigation of the wrapping process. A detailed reconstruction of a representative growing myelin sheath was realized from the axon pointed by a red arrow (see Fig 3.4). Scale bar $1 \mu \mathrm{m}$. 
A

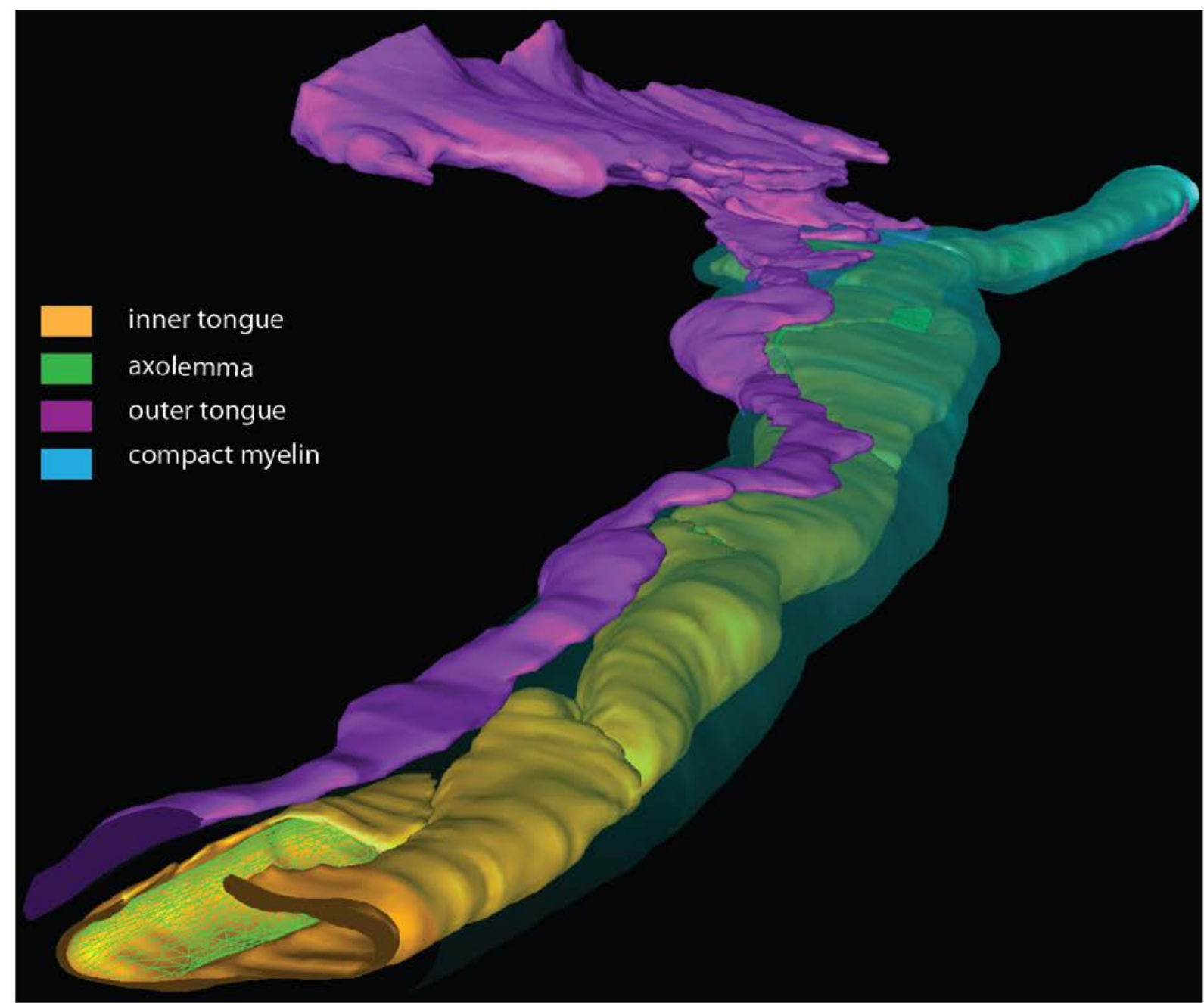

B

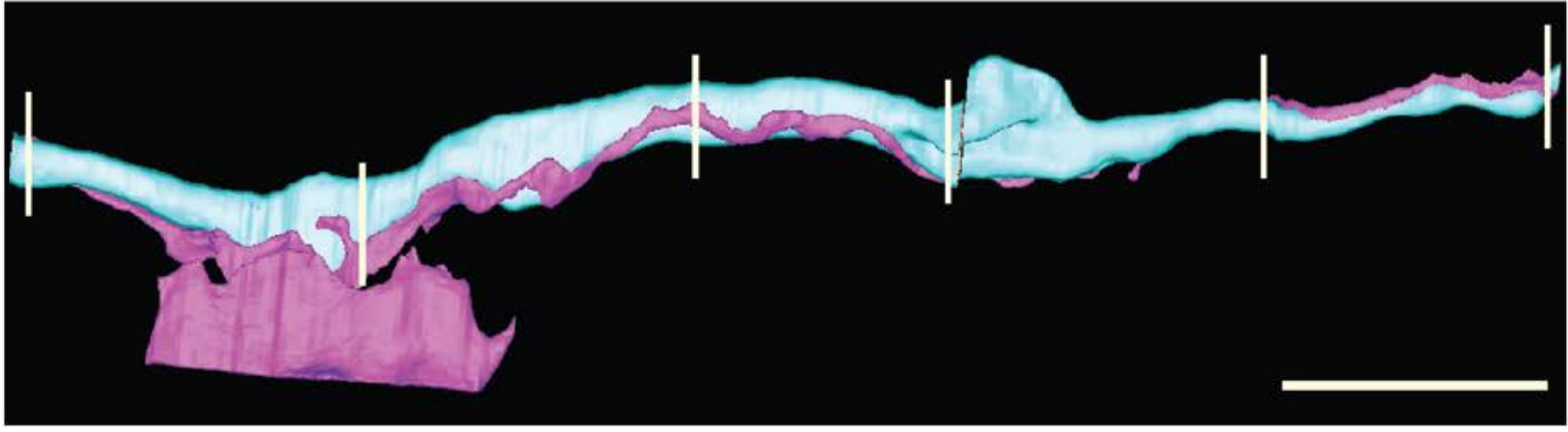

C

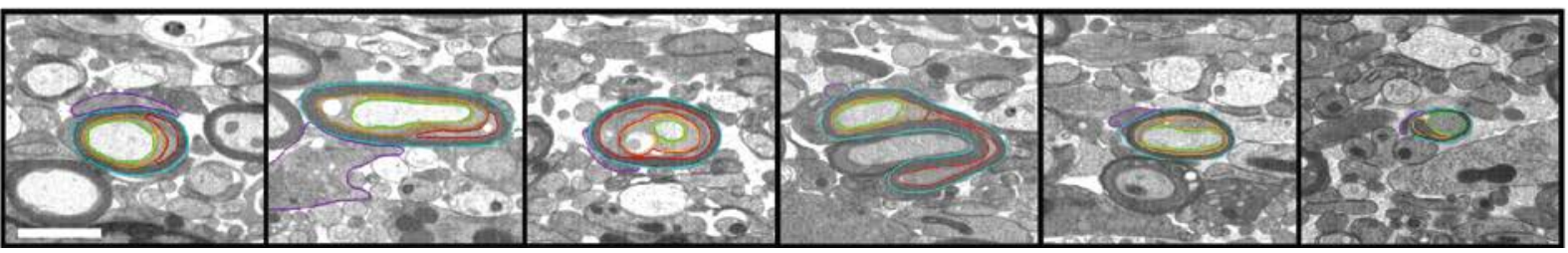




\section{Figure 3.4: Investigation of the myelin ultrastructure of a growing sheath}

A) Three dimensional reconstruction of a representative P10 myelination pattern along $60 \mu \mathrm{m}$. The outer tongue and the oligodendrocyte cell body are represented in violet, the compact myelin sheath in cyan, the inner tongue in orange and the axon in green. B) Surface view of the modeled myelin sheath with its corresponding electron micrographs along the growing sheath. The areas where the electron micrographs were taken are marked from a-f to illustrate the difference in thickness and morphology of the sheath along a hemi-node. The red traces mark the cytoplasmic channels (details of these structures in chapter 3.4.1). Scale bar $10 \mu \mathrm{m}$ for the model and $1 \mu \mathrm{m}$ for the electron micrographs.

The quantification of the winding frequency of these two tongues based on $453 \mathrm{D}$ reconstructions is in average $5.9 \mu \mathrm{m} \pm 0.64$ for the inner tongue and $70.9 \mu \mathrm{m} \pm 8.0$ for the outer tongue (fig 3.5). The inner tongue would wind up to the level of the oligodendrocyte process and would unwind towards the other end. Thus, the thickest myelin is located in the middle of the growing segment and possibly in the front of the oligodendrocyte process.

The data obtained by the FIBSEM acquisitions allowed to successfully combine the high resolution needed to resolve the morphology of the myelin sheath at nanometer range and the large volume required to address myelination in vivo. Based on these readings we could address the positioning of the cytoplasmic rich areas along the myelin segment during axonal ensheathment. 


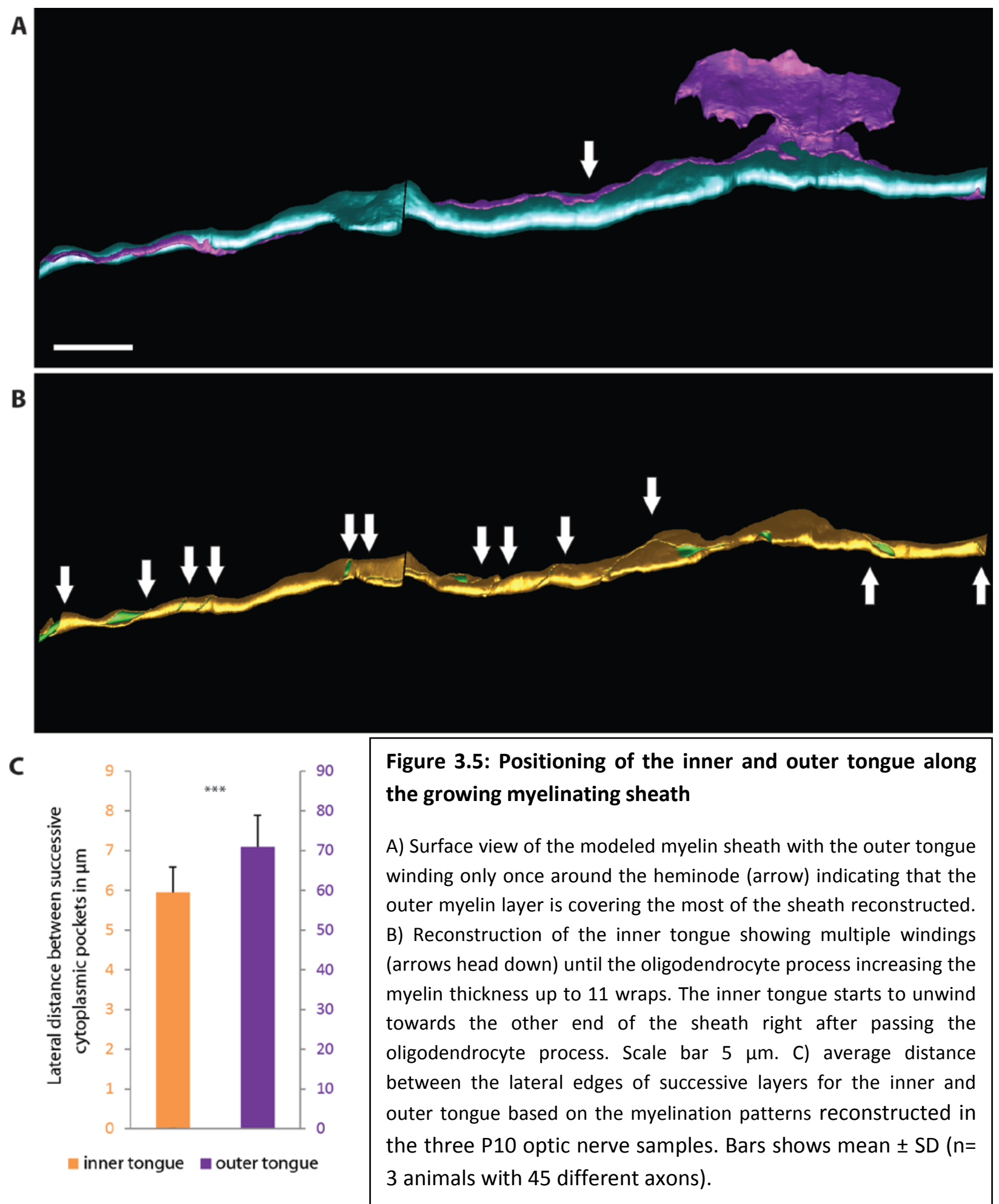




\subsubsection{Investigation of the myelin layer organization in the growing myelin sheath}

Conjointly with the FIBSEM we investigated the myelin layer organization in growing myelin sheath using transmission electron microscopy (TEM). Indeed, in addition to the larger amount of samples assessed with the TEM due to the direct availability of the microscopes, the resolution achievable with the TEM is greater than the one of the FIBSEM. Indeed in contrast to the FIBSEM, the resolution of individual myelin layers within the compacted sheath was always possible using the TEM.

Based on longitudinal sections of high pressure frozen P10 optic nerves we could resolve single layers in the compact myelin as well as cytoplasmic rich areas and could correlate it with the spatial morphology of the cytoplasmic rich regions obtained with the FIBSEM. When following the developing myelin sheath along the axon, we could confirm the progressive and constant reduction of the myelin thickness towards the end of the segments (fig 3.6, video 03).
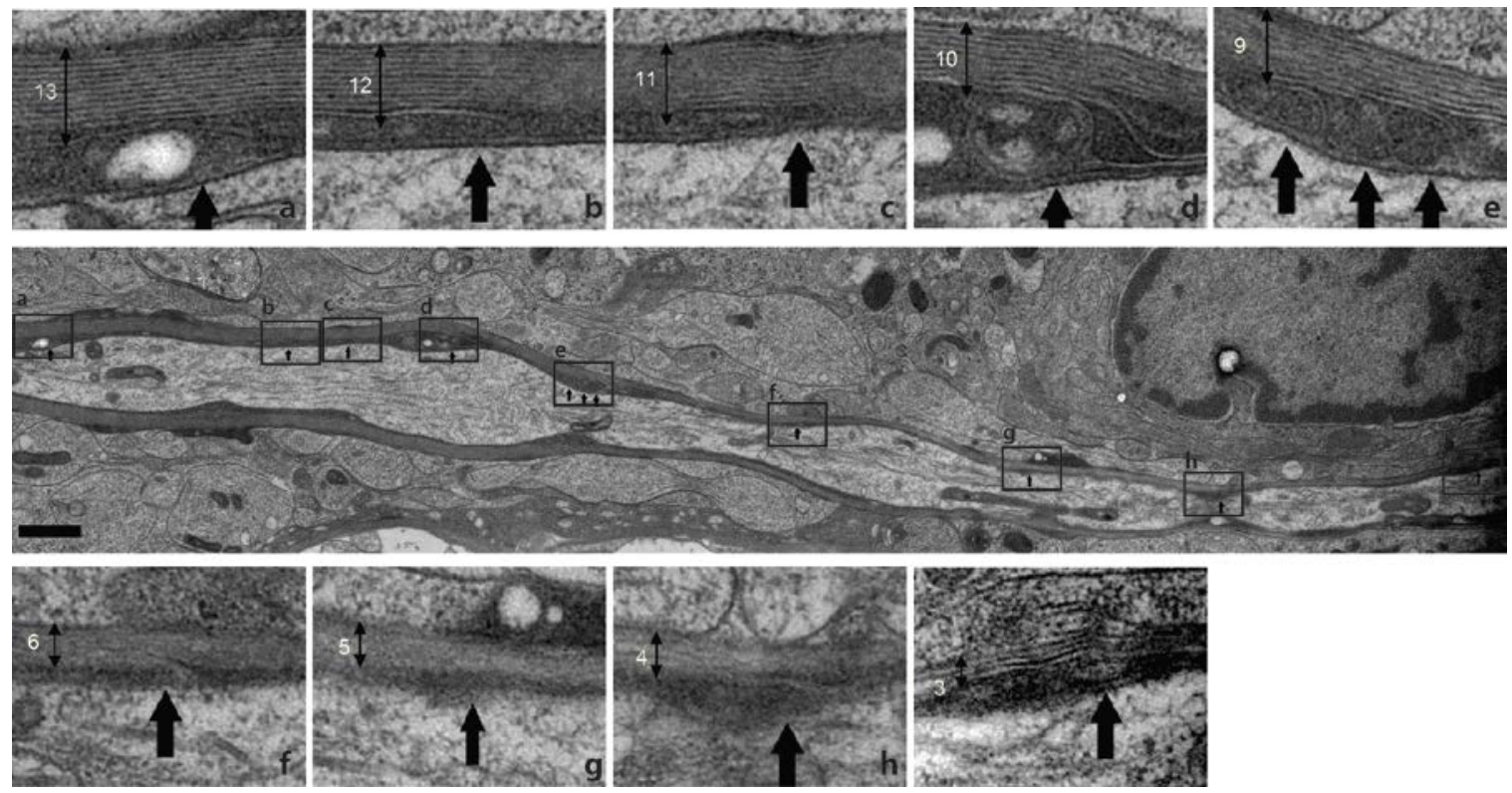

Figure 3.6: Positioning of the successive myelin layers in a growing myelin sheath by TEM

Longitudinal section of a P10 optic nerve showing a growing myelin sheath obtained by high pressure freezing. The morphology of each successive layer can be followed from a 13 wraps thick myelin down to 3 wraps. Along the segment each layer edge is pointed by a black arrow and happens invariably at the inner most wrap of the myelin. From a) to i) are enlarged views of the edges of the successive wraps. Scale bar $1 \mu \mathrm{m}$. 
Also, according to the FIBSEM data we confirmed that the outermost layer was uninterrupted along the myelin segments (60 segments of $20 \mu \mathrm{m}$ in average, from 3 different animals). The myelin thinning along the growing myelin sheath appeared invariably from the successive interruption of the myelin layers facing the axon (fig 3.6). In addition, we found that in all the samples analyzed the myelin layers of the sheaths were uninterrupted between their cytoplasm-rich edges confirming the notion that the growing myelin sheath is composed of a single plasma membrane extension (movie). Based on these longitudinal sections in the internodal portion, we quantified the distance between the lateral cytoplasmic-rich edges of the successive layers and found that at P10 the average spacing was around $5.2 \mu \mathrm{m} \pm 0.1$ (fig 3.7). At $P 21$ we found that the average spacing between successive cytoplasm-rich endings increased to $34.2 \mu \mathrm{m} \pm 4.2$ and finally at P60 would reach $137.3 \mu \mathrm{m} \pm 15.4$ (fig 3.7). Correspondingly, when assessing the paranodal regions of developing myelin sheath at P10, P21 and P60, we could see that the distance between the successive cytoplasm-rich edges that correspond to the paranodal loops, would continuously decline from $2.5 \mu \mathrm{m} \pm 0.1$ at $\mathrm{P} 10,0.3 \mu \mathrm{m}$ \pm 0.04 at $\mathrm{P} 21$ to $0.2 \mu \mathrm{m} \pm 0.03$ at $\mathrm{P} 60$ (fig 3.8).

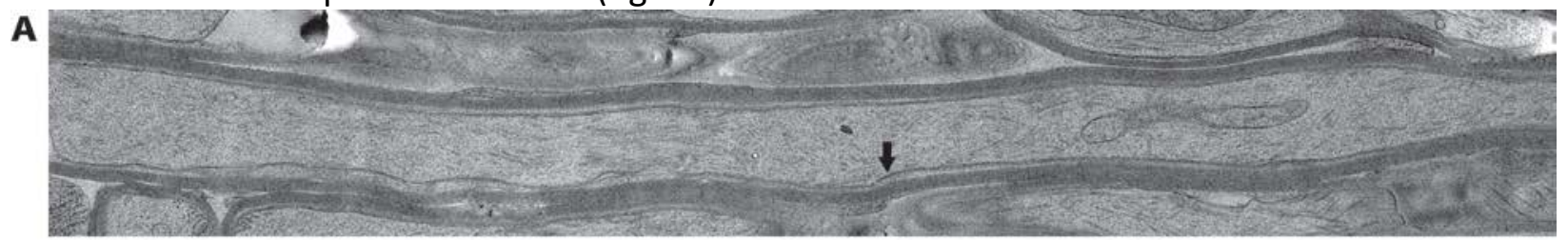

B

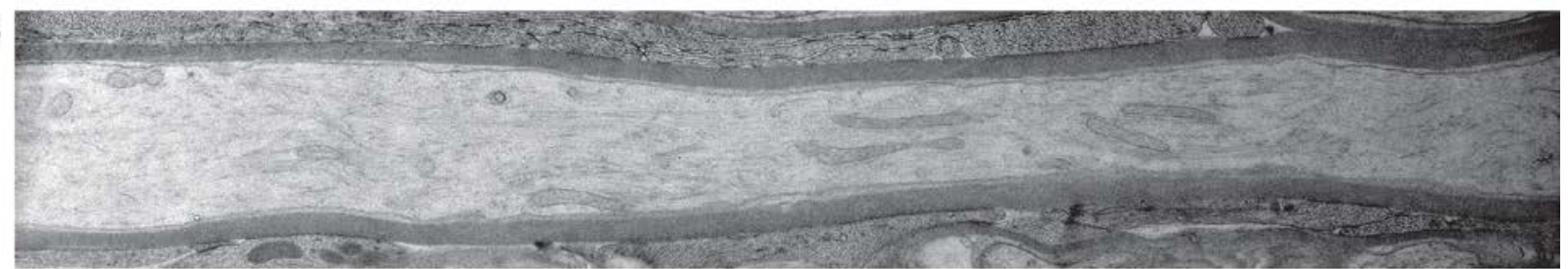

C

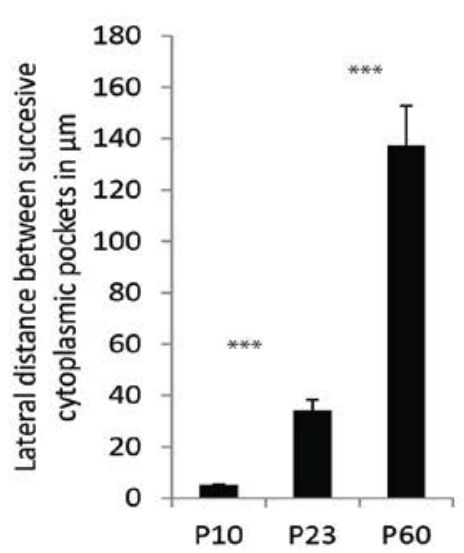

Figure 3.7: Evolution of the internode morphology during CNS myelination

Longitudinal section of myelinated axon at P21 (A), showing a seldom interruption of the inner tongue (arrow), and at P60 (B) with no interruption along the segment. C) Average distance between the lateral edges of successive layers measured from TEM longitudinal sections of P10, P21 and P60 optic nerve samples. Bars shows mean \pm SD ( $n=3$ animals with 20 longitudinal myelinated axons). Scale bar 1 $\mu \mathrm{m}$. 


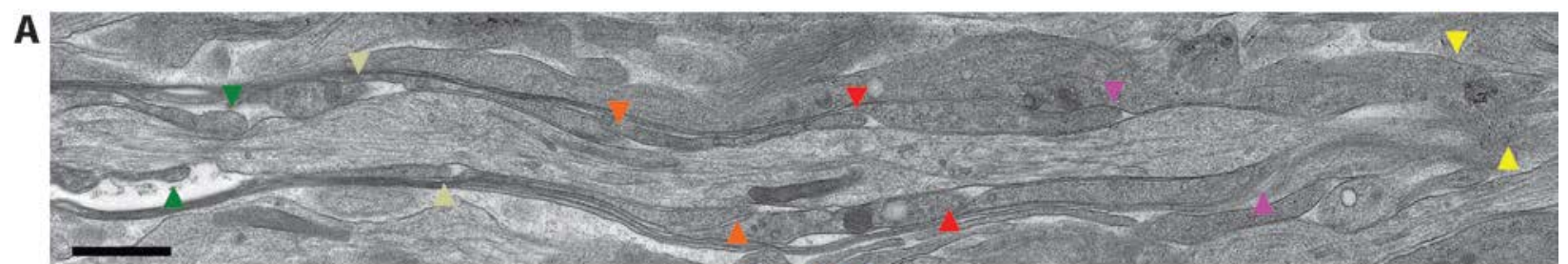

B

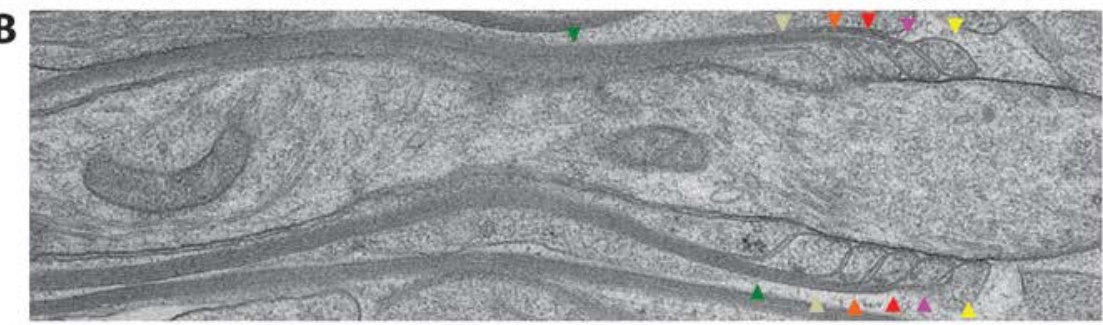

C

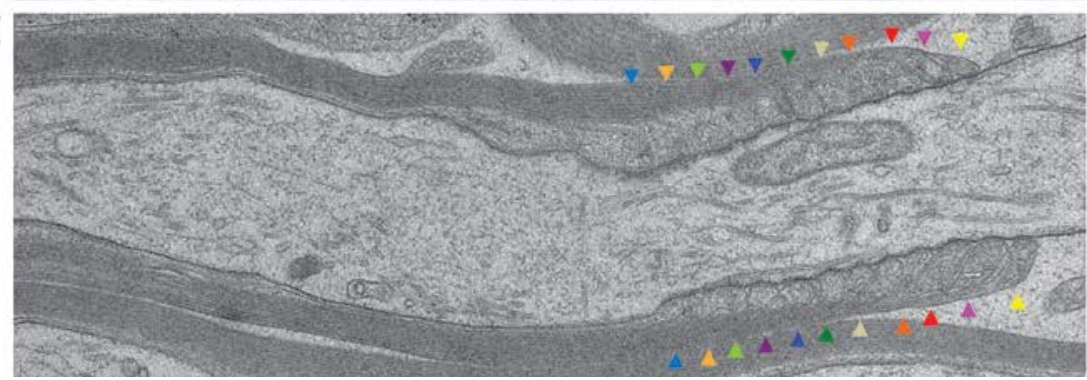

D

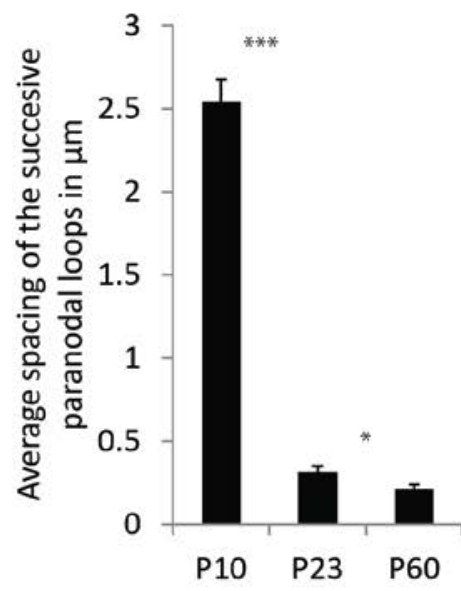

Figure 3.8: Evolution of the node morphology during CNS myelination

Longitudinal section of the lateral ends of the myelinated segments at P10 (A), P21 (B) and P60 (C) showing a reduction in the space between successive layer's edges pointed with different colors (future paranodal loops). D) Average distance between the lateral edges of successive layers measured at the edges of the myelin segment from TEM longitudinal sections of P10, P21 and P60 optic nerve samples. Bars shows mean \pm SD ( $n=3$ animals with 20 longitudinal myelinated axons). Scale bar $1 \mu \mathrm{m}$.

Finally, we investigated the global morphology of the myelin sheath by TEM and verified our previous observations along a full internode during myelin growth in the optic nerve. Using longitudinal serial sections we could reconstruct a longitudinal cut through a full internode of P10 optic nerve where each myelin layer could be followed from one to the other end of the segment. First we could see that the myelin sheath was the thickest at the level of the oligodendrocyte process and that the outer layer was continuous and covering the entire internode (fig 3.9). Each successive myelin layer towards the axon (marked with a different color) would be smaller in width, centered to the oligodendrocytic process and uninterrupted from one lateral cytoplasm-rich edge to the other (fig 3.9).

Based on these TEM data we could confirm the morphology observed by FIBSEM by identifying the lateral cytoplasm-rich edges of the layers as well as the continuity of the layers along the 
internode. Furthermore we could address the positioning of the layers at three different time points in development and see that the sheath would develop from a triangular shape in early stages to a rectangular sheath once mature.

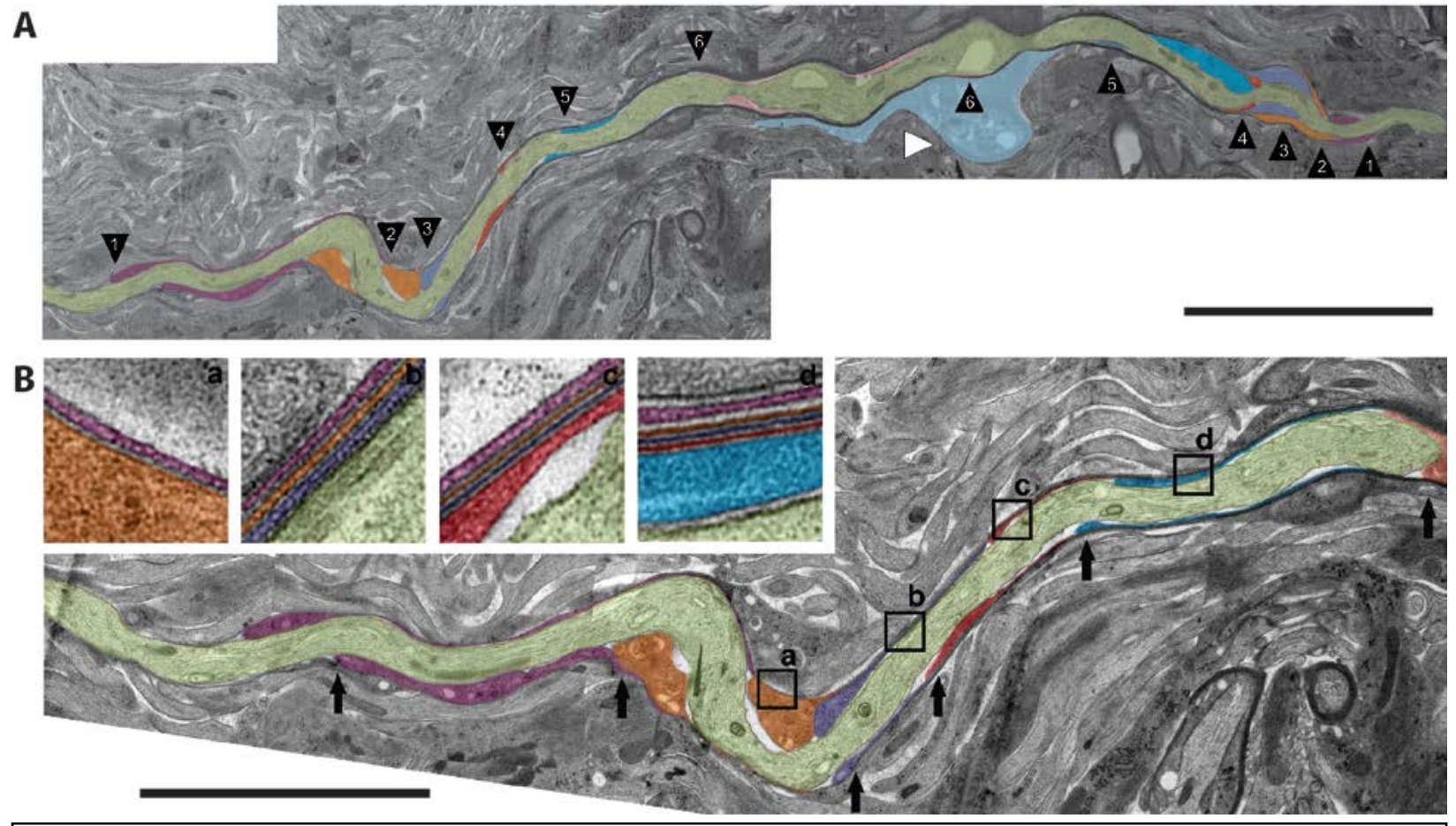

Figure 3.9: Organization of the successive myelin layers along a full internode during myelination

A) Montage of longitudinal serial sections of P10 optic nerve showing the successive myelin wraps in different colors spreading along the axon with the outer most layer covering the full length of the sheath and the inner most layer been the shortest centered in front of the oligodendrocyte process (white arrow). The edges of the successive layers are pointed by a numbered black arrows from the most superficial to the inner most wrap. B) Enlarged view of the myelin segment with blown-ups showing the colored successive layers running all along the sheath. Scale bar $5 \mu \mathrm{m}$ 


\subsubsection{In vivo motion of the growing myelin layers by live imaging in Zebrafish}

The morphological investigation of the growing myelin sheath with electron microscopy allowed us to visualize the myelin at nanometer range, but due to the sample preparation required by this technique the dynamic aspects of the myelination were not assessable. To investigate the changes occurring in a growing myelin sheath in the matter of minutes or hours, we used zebrafish as model organism based on its amenability for high resolution in vivo imaging and its emergence as a suitable model for myelination studies (Buckley et al., 2008). The recordings used in this study were acquired in collaboration with Tim Czopka and David A. Lyons in the Centre for Neuroregeneration, University of Edinburgh, UK.

We investigated the initial events of CNS wrapping using the $\operatorname{Tg}(n k \times 2.2 a: m e G F P)$ animal line, which expresses a membrane-tethered GFP in a subset of oligodendrocyte precursor cells and early myelinating oligodendrocytes (Kirby et al., 2006). We could visualise the formation and development of the first myelin wraps by recording the fluorescence intensity along 32 nascent myelin sheaths at 3-4 days post fertilisation. We found that the intensity of the fluorescence was not constant along the segment and was consistently higher at the centre of the nascent sheath (fig 3.10, video 04). Furthermore, we could see that the variations in fluorescence along the segments were occurring almost always in a quantal steps (fig 3.10) suggesting that this variation would reflect the increase or decrease in the amount of layers. It is important to mention that the myelin sheaths are mostly not compacted at that stage as confirmed by the EM imaging (fig 3.10). Indeed, based on our previous observations (Aggarwal et al., 2011); (Aggarwal et al., 2013) we know that GFP is extruded from the compact myelin upon MBP compaction, but until this process starts it is possible to use GFP as a marker of the myelin membrane. We made use of these observations to follow the first steps for the wrapping processes in vivo in a time lapse imaging over several hours and found that first all the myelin segments would grow laterally in a matter of minutes to hours (fig 3.11). Furthermore, we could observe that the quantal steps present along the segment would also move laterally towards the end of the myelin sheath often faster than the edges of the internode (fig 3.11). To complement these observations we analysed a second line of GFP mutant; the $\operatorname{tg}(\mathrm{mbp}$ :EGFPCAAX) where the GFP fluorophore was driven by the MBP promoter. 
A
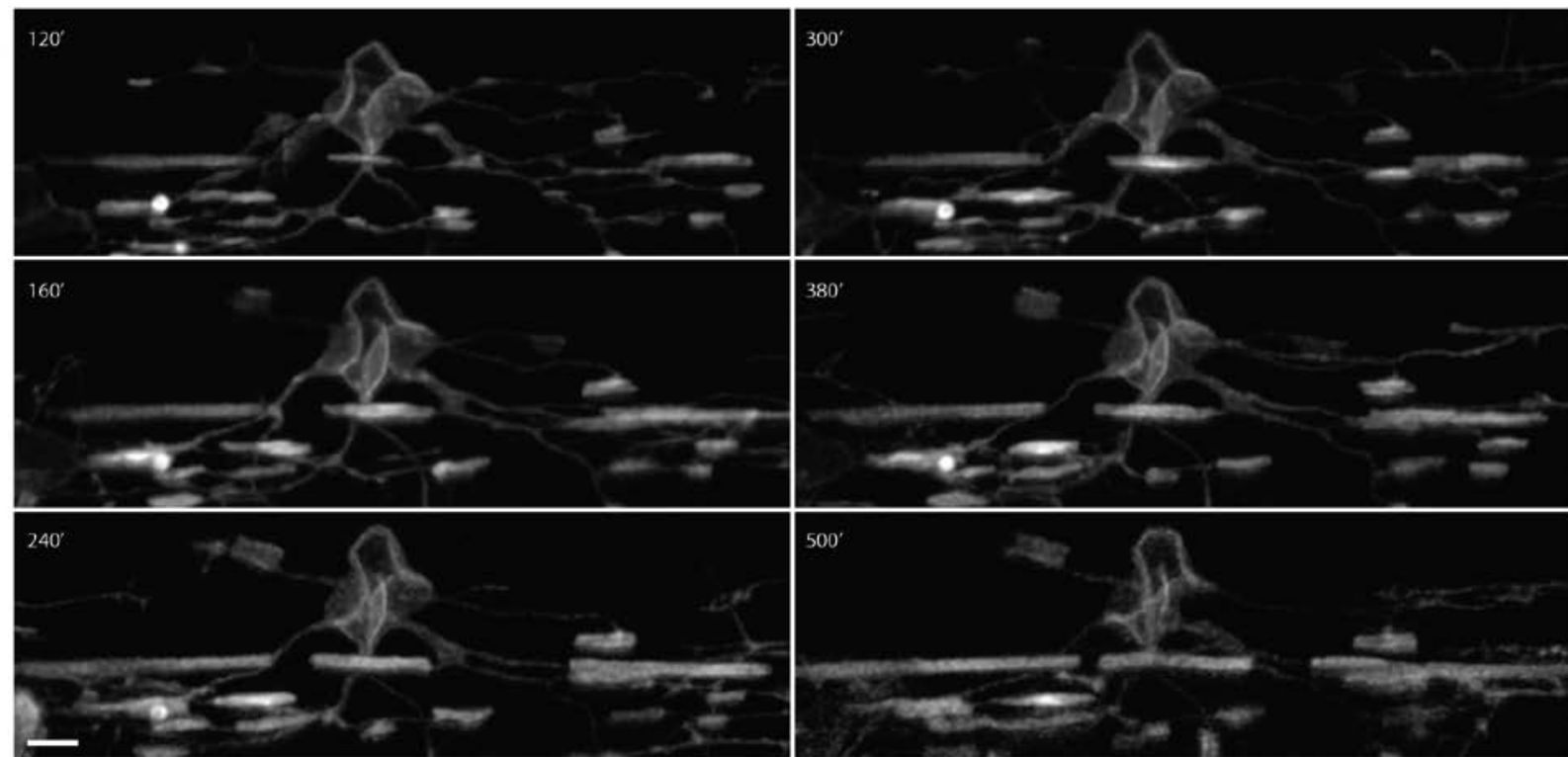

B

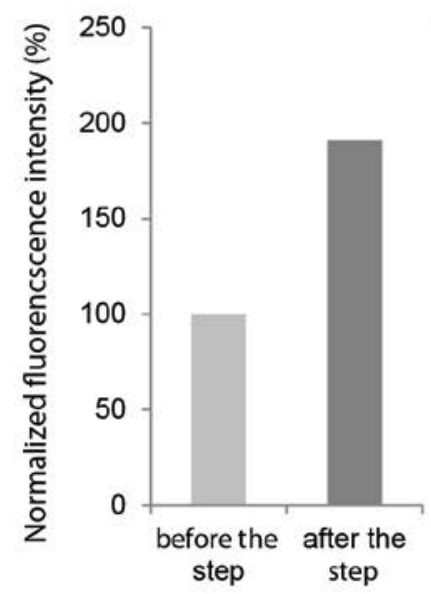

C

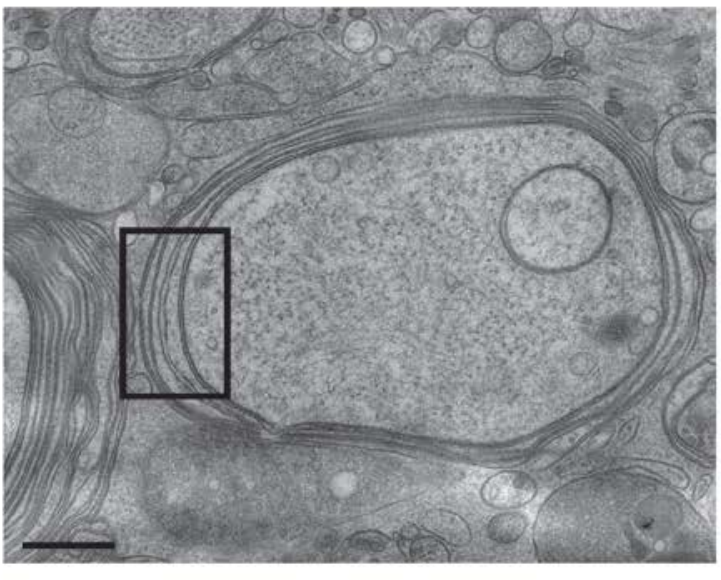

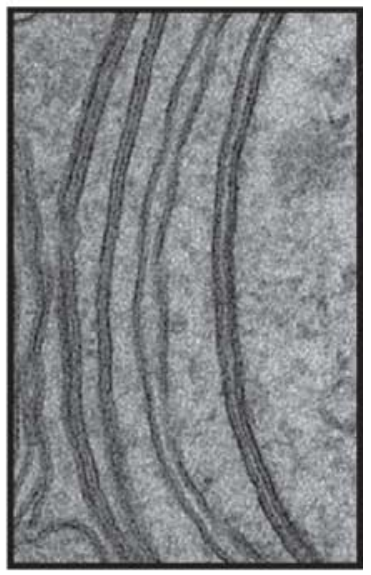

D

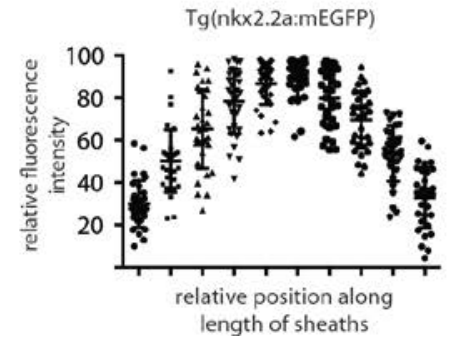

\section{Figure 3.10: Live imaging of myelination events in Zebrafish}

A) Live imaging of Zebrafish spinal cord of the $\mathrm{Tg}(\mathrm{nkx2.2a}$ :meGFP) at 3 days post fertilization (dpf) for 500 minutes. Scale bar $2 \mu \mathrm{m}$. B) Average intensity variations in the fluorescence steps in early myelinating sheaths measured from 14 sheaths in 3 different animals. C) Electron micrograph at 3-4 days post fertilization showing non-compacted intra-cytoplasmic space. Scale bar $500 \mathrm{~nm}$. D) Average of the relative fluorescence along the myelin sheath of 34 sheaths in 3 different animals of the $\operatorname{Tg}(\mathrm{nkx2}$.2a:meGFP) lines. Bars shows mean $\pm \mathrm{SD}$. 
With this mutant line we could observe the distribution of the fluorescence in later stage of myelin sheath maturation when the segments became far longer: around $40 \mu \mathrm{m}$ against $10 \mu \mathrm{m}$ for the $\operatorname{Tg}(n k x 2.2 a: m e G F P)$ line (fig 3.11).We found that the fluorescence intensity profiles along the length of these more mature myelin segments were more complex and at the same time, more uniform when considering the length of the sheath (fig 3.11). This is probably due to the greater amount of layers that compose these sheaths and the extrusion of the membrane tethered GFP from the compact myelin (fig 3.11). Indeed, in previous studies we could show that the GFP tagged proteins are going to be sequestered to the non-compacted cytoplasmic areas at the inner and outer tongue upon myelin compaction (Aggarwal et al., 2011); (Aggarwal et al., 2013).
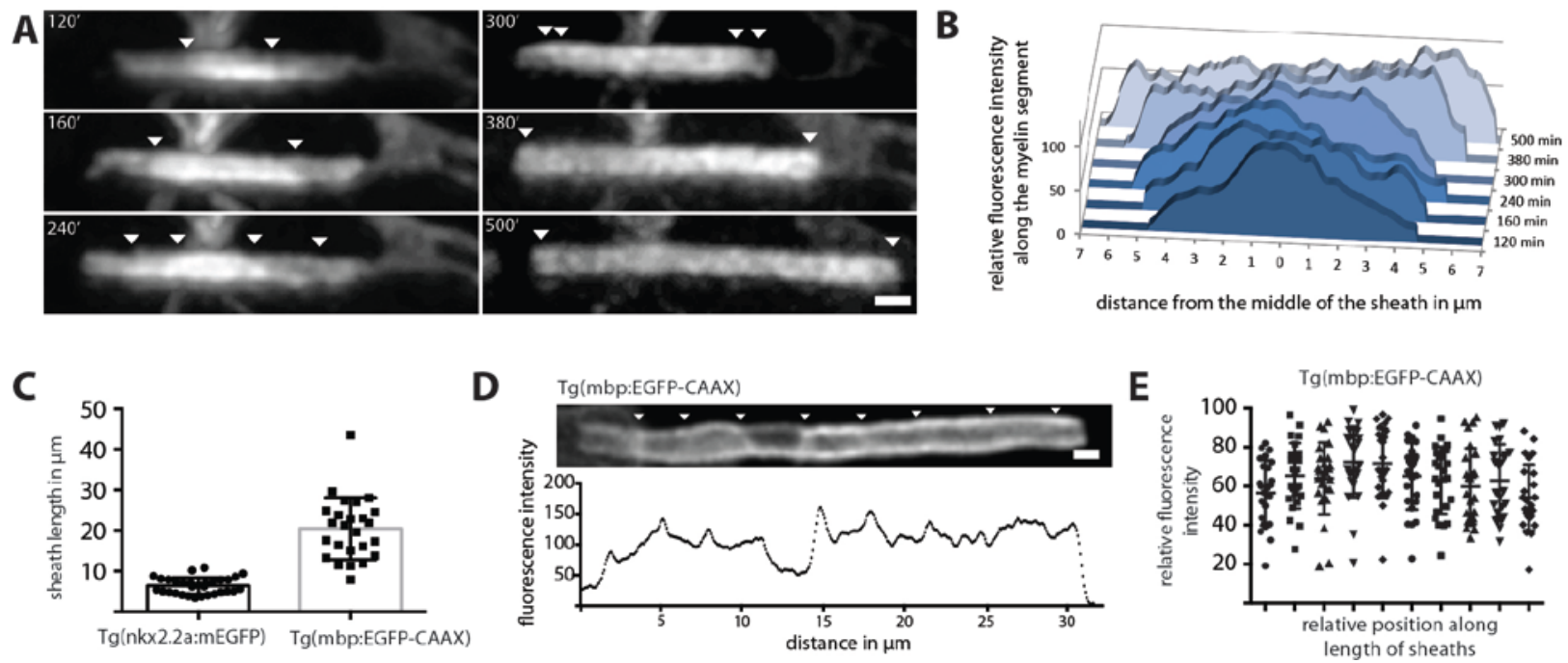

\section{Figure 3.11: Lateral extension of myelin layers}

A) Live time visualization of the growth of a myelin segment for 500 minutes where the step in fluorescence are pointed with arrows. B) Graphic representation of the relative fluorescence and its evolution in time along the myelin segment shown in (A). C) Graphic representation of the average segment length between the line $\operatorname{Tg}(\mathrm{nkx2}$.2a:mEGFP) expressing GFP at an early time point of myelination and the $\operatorname{Tg}(\mathrm{mbp}$ : EGFP-CAAX) line expressing EGFP when the compaction by MBP is achieved. E) Lateral view of a $4 \mathrm{dpf}$ spinal cord in $\mathrm{Tg}(\mathrm{nkx2.2a}$ :mEGFP) that illustrate the fluorescence pattern along the maturing myelin segment. Arrows are pointing at areas where the fluorescence intensity is the highest. F) Average of the relative fluorescence along

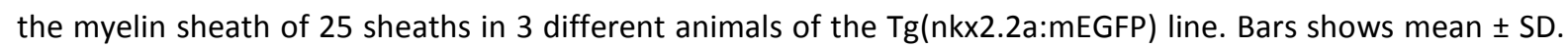
canlaham 1...m 
Based on the characterization of individual layer morphology acquired with the TEM observations, the spatial organization of the cytoplasmic-rich domains by FIBSEM 3D reconstruction and the motion of the layers by live imaging in vivo we could propose a model where an advancing inner tongue is winding around the axon in the center of the segment. This motion would allow the radial growth of the myelin sheath. Concurrently to this first growth motion the successive layers would extend laterally towards the future location of the node of Ranvier. These observations correspond to our previous data where we found that the center of the myelin sheath is the thickest and the myelin thinning toward the node appeared invariably from the successive interruption of the myelin layers facing the axon. Furthermore, this model would explain not only the minor winding of the outer tongue and the far greater winding of the inner tongue, but also the lateral extension of the layers observed in the live imaging with the $\operatorname{Tg}(n k x 2.2 a: m e G F P)$ zebrafish line and the coiling patterns within the maturing sheath of the $\operatorname{Tg}(\mathrm{mbp}$ :EGFP-CAAX) line. 


\subsection{Localization and characterization of the oligodendrocyte/myelin growth zone in vitro and in vivo.}

Our morphological investigation could provide a clear understanding of the architecture of the growing myelin sheath and its motion during the maturation. However, the molecular mechanisms underlying the growth were not possible to address with such techniques. Furthermore, the identification of the zone where the newly synthesized material is added to the sheath has not yet been described and defined. Due to obvious accessibility limitations of nervous system tissues and the time needed for in vivo myelination we used a previously described model of primary oligodendrocyte in culture (Trajkovic et al., 2006) to identify the growth zone and the molecular mechanisms leading to the polar growth of the myelin sheath of the oligodendrocyte.

\subsubsection{Tracking the plasma membrane trafficking in cultured oligodendrocytes using the vesicular stomatitis virus}

Our objective was to define and characterize the zone where the newly synthesized material is added in the oligodendrocyte during CNS myelination. To visualize the zone at which the proteins and lipids are integrated into the growing myelin, a large amount of membrane associated reporter needed to be generated in a short time to limit the effect of the diffusion on the imaging. Therefore we used a well-established plasma membrane trafficking reporter: the vesicular stomatitis virus glycoprotein G (VSV-G) to address this question. The VSV-G is a trans-membrane class III viral fusion protein that is known to be transported via the basolateral sorting machinery (de Vries et al., 1997).

We infected primary cultures of oligodendrocytes with VSV to assess the membrane trafficking in a system easy to access and monitor. Each of the cell culture coverslips of $13 \mathrm{~mm}$ was infected with $5 \times 10^{5} \mathrm{pfu} / \mathrm{ml}$ of virus for 15 minutes, then the free floating viruses were removed by washing the cells with fresh medium and the cells were further incubated for 2,3,4 and 7 additional hours before being fixed and further proceeded for immunofluorescence. We then assessed the distribution of the VSV-G on the surface and within the oligodendrocytes using two approaches. First, by permeabilizing the cell prior to the antibody binding we would label the total amount of VSV-G in the cell (total staining). 

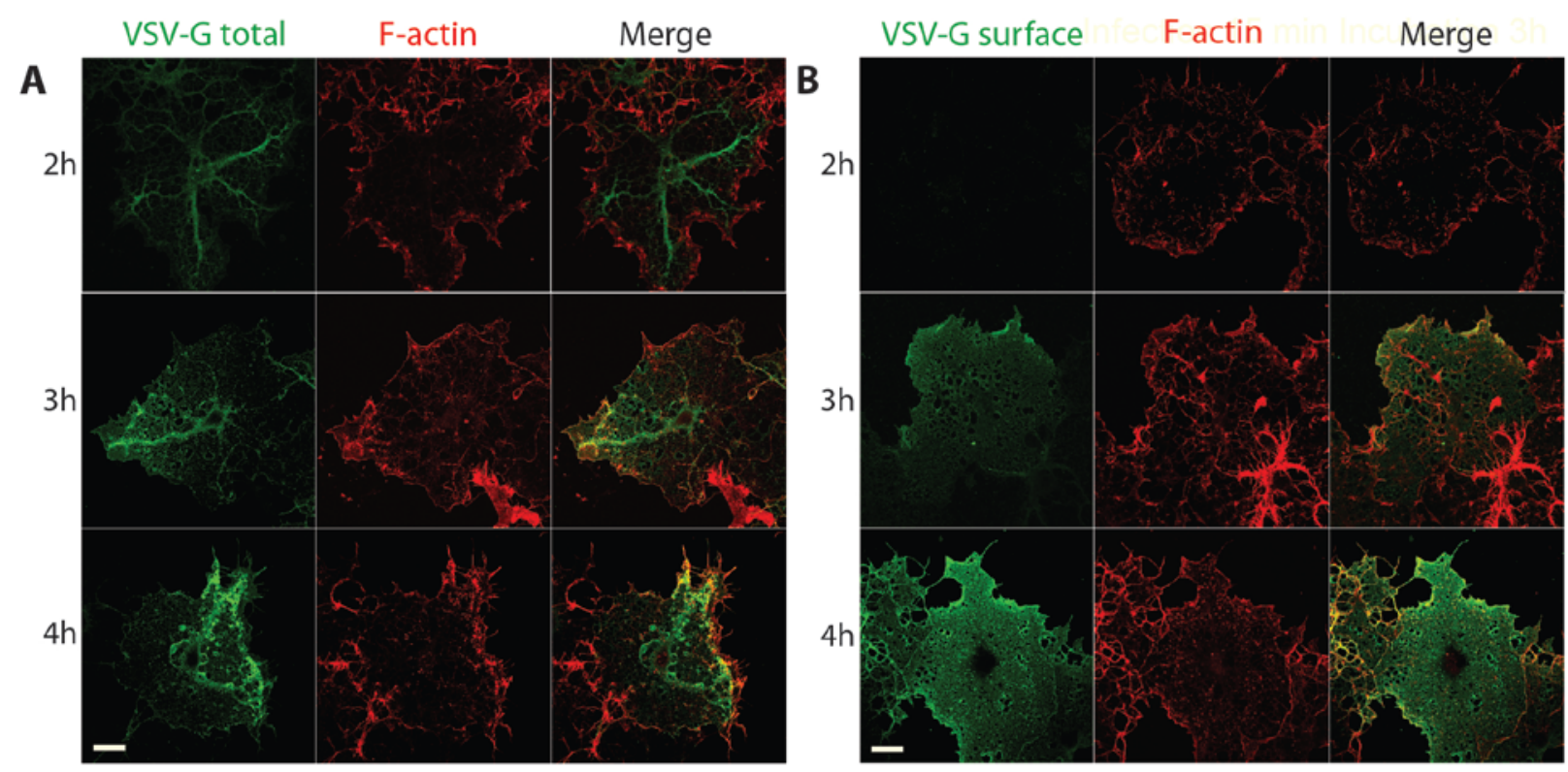

C
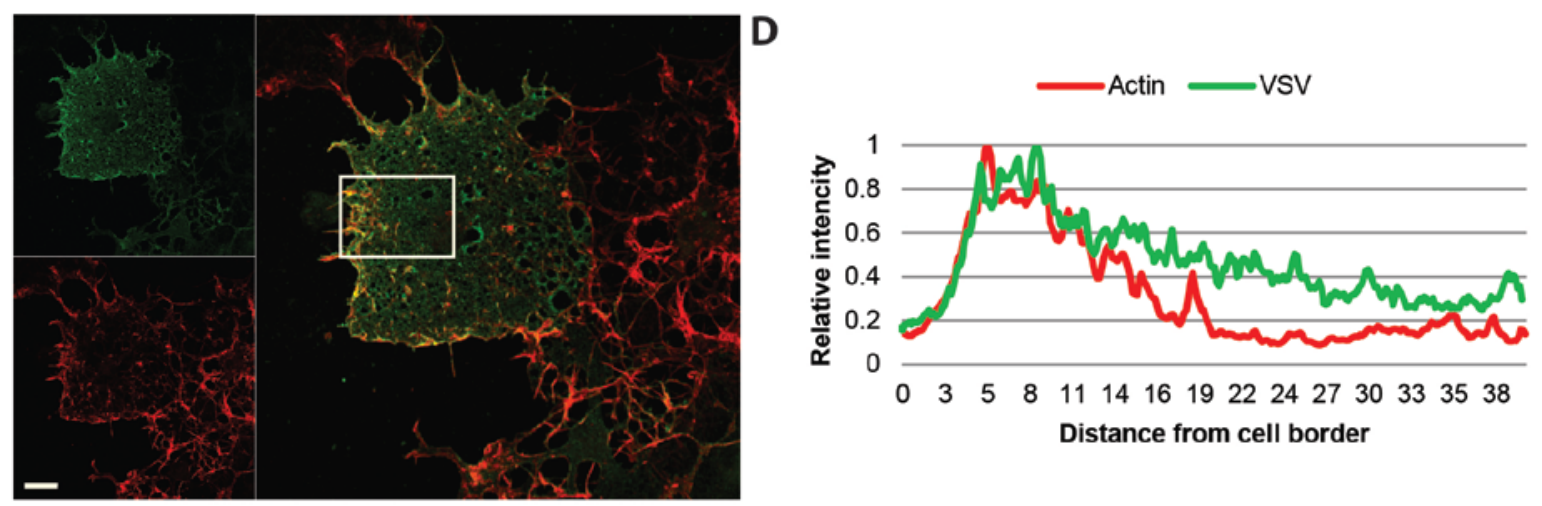

E
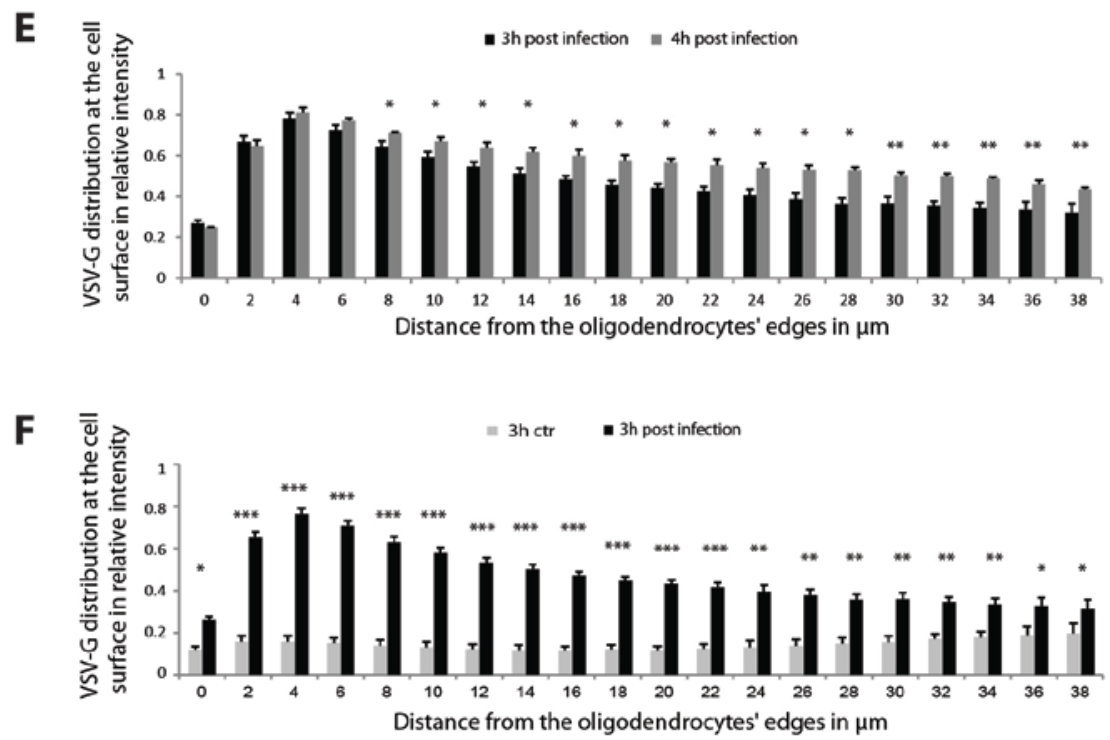


\section{Figure 3.12: Tracking the membrane trafficking using the vesicular stomatitis virus $\mathrm{G}$ protein in oligodendrocyte cell culture.}

A and B) Total and surface staining of VSV-G in cultured primary oligodendrocytes infected by the VSV for 2, 3 and 4 hours. The fluorescence can be found in cytoplasmic compartments starting from 2 hours post infection and at the cell surface starting from 3 hours on, primarily at the cellular rim. C) Area of $20 \mu \mathrm{m}$ by $40 \mu \mathrm{m}$ spreading from the cellular rim towards the cell body selected to quantify the VSV-G surface staining of cultured oligodendrocytes infected by VSV. D) Graphic representation of the actin and VSV-G intensity within the selected area shown in C). E) Average relative fluorescence intensity of VSV-G at the cell surface 3 and 4 hours after infection using the same region of interest described in (C) on 3 independent cultures, 30 cells per culture. F) Average relative fluorescence intensity of VSV-G at the cell surface 3 hours after infection compared to control cells. Bar show mean $\pm S D$ ( $n=3$ independent cell cultures, 30 cells per culture). Scale bars $20 \mu \mathrm{m}$.

In a second approach we would allow the antibodies to bind to the non-permeabilized cells labeling only the molecules that were transported to the growth zone and incorporated to the plasma membrane (surface staining).

At 2 hours post infection the signal obtained by the total staining was almost exclusively within the cell bodies and the cytoplasmic processes connecting the cell body, when no surface staining could be observed (fig 3.12). At the later time points, 3 and 4 hours post infection, in addition of the cytoplasmic processes, the cellular rim became heavily stained with the total staining (fig 3.12).

Using the surface staining and starting from 3 hours after infection a clear gradient of VSV-G was observable from the cellular rim of the oligodendrocytes towards the cells body. Interestingly we found that $\mathrm{f}$-actin was enriched at the rim of the oligodendrocyte in culture (fig 3.12). The distribution of the f-actin and surface VSV-G labeling was projected from a $20 \mu \mathrm{m}$ wide region of interest (ROI) along $40 \mu \mathrm{m}$ towards the cell body (fig 3.12). We observed a constant VSV-G pattern at 3 and 4 hours post infection with most of the labeling being localized at the cell rim and rapidly fainting towards the cell body. We also measured the f-actin distribution within this area and found that the signal was concentrated within the first 10-12 micrometer from the cell rim indicating the non-compacted area of the cellular rim (fig 3.12). We could observe that the VSV-G enrichment at the cellular rim became less pronounced at 4 hours compared to 3 hours post infection indicating the diffusion of the VSV-G in the plasma membrane from the cellular rim towards the cell body over time (fig 3.12). Conjointly to the immunofluorescence we carried out immune electron microscopy for VSV-G on cultured oligodendrocytes at 2 and 4 hours after infection. At 2 hours post infection we could visualize 
the subcellular localization of the labeling almost exclusively in the Golgi apparatus and in vesicular profiles (fig 13). However at 4 hours post infection in addition to the Golgi and vesicular profiles, the membrane would show a dense VSV-G labeling (fig 3.13) confirming our light microscopy observation.
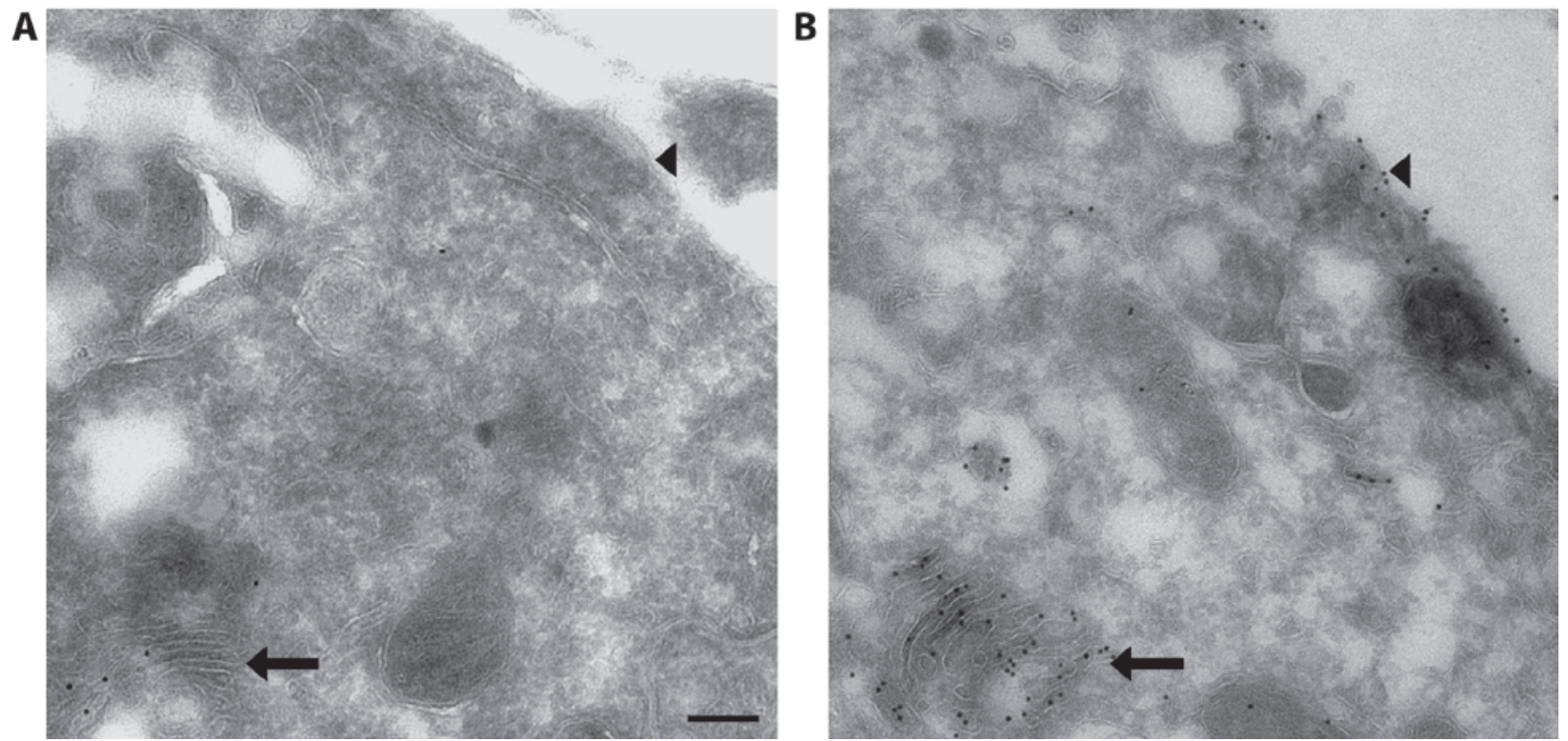

Figure 3.13; Subcellular localization of the VSV-G in cultured oligodendrocyte

A) Immuno electron micrograph of oligodendrocyte in culture infected for 2 hours with VSV and stained for VSV-G. The labeling can be found almost exclusively at the Golgi apparatus (arrow). B) At 3 hours post infection the labeling intensity is higher and the VSV-G labeling can be found at the Golgi apparatus (arrow) but also at the plasma membrane (arrow head). Gold size particles $10 \mathrm{~nm}$. Scale bar $200 \mathrm{~nm}$

Our analysis on the total and surface VSV-G staining distribution revealed interesting features that gave us more insides about the trafficking to the plasma membrane in cultured oligodendrocytes. For example, when the cells were be in contact with neighboring oligodendrocytes no VSV-G signal could be seen neither in the cytoplasmic processes leading to the rim towards this area nor on the cell surface (fig 3.14). Furthermore, we could establish a clear correlation between the F-actin rim size and the VSV-G distribution in the plasma membrane. When the F-actin was almost not detectable at the cellular rim of the cultured oligodendrocytes, there was also less VSV-G surface staining in these areas (fig 3.14). We also found that the compaction state of the oligodendrocyte would influence neither the polarity of the labeling (fig 3.14) nor the diffusion of the VSV-G proteins at the surface of the cell (fig 3.14). 
A

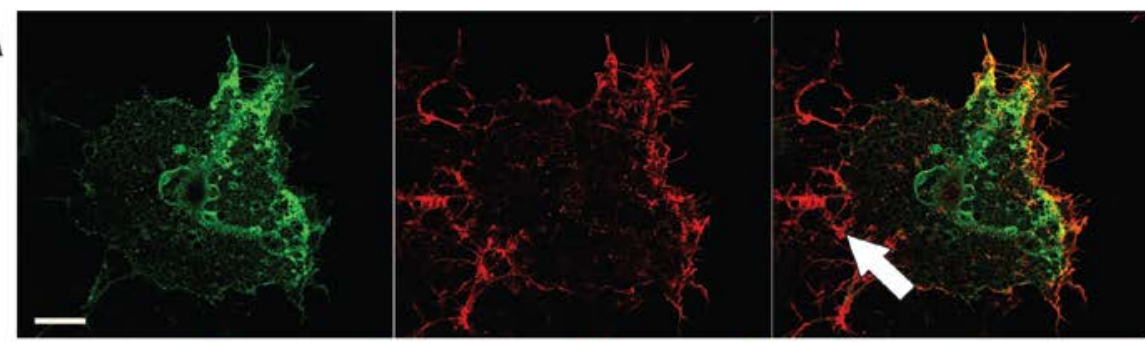

B

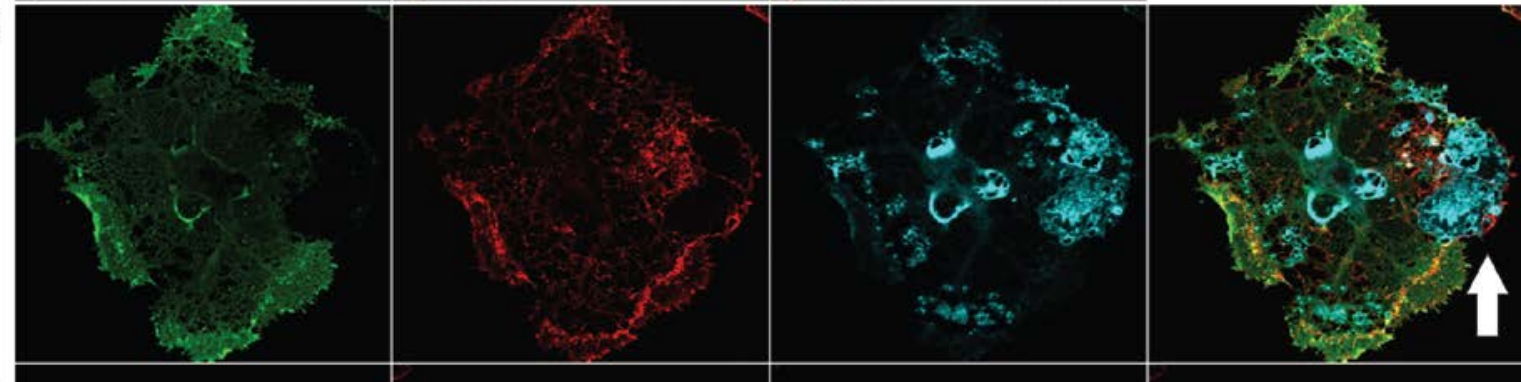

C

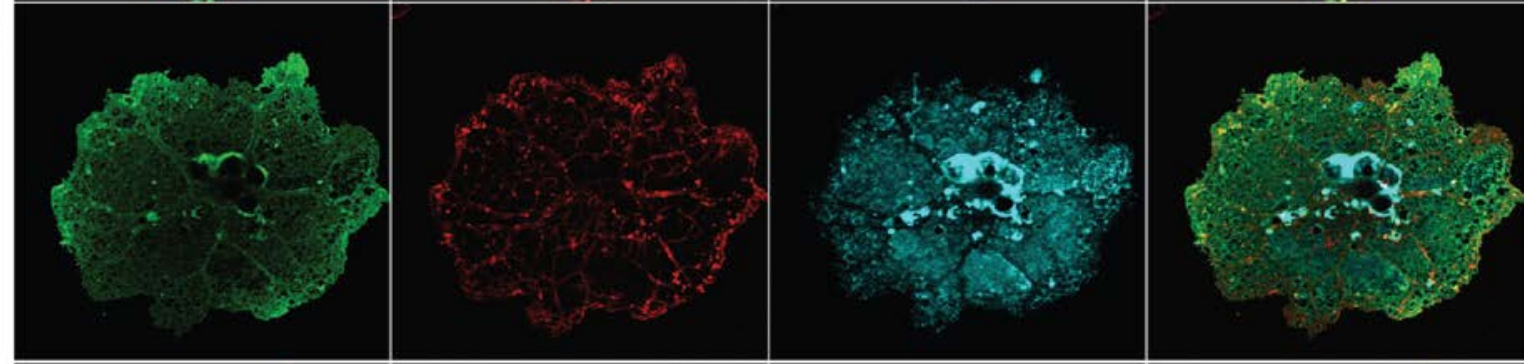

D

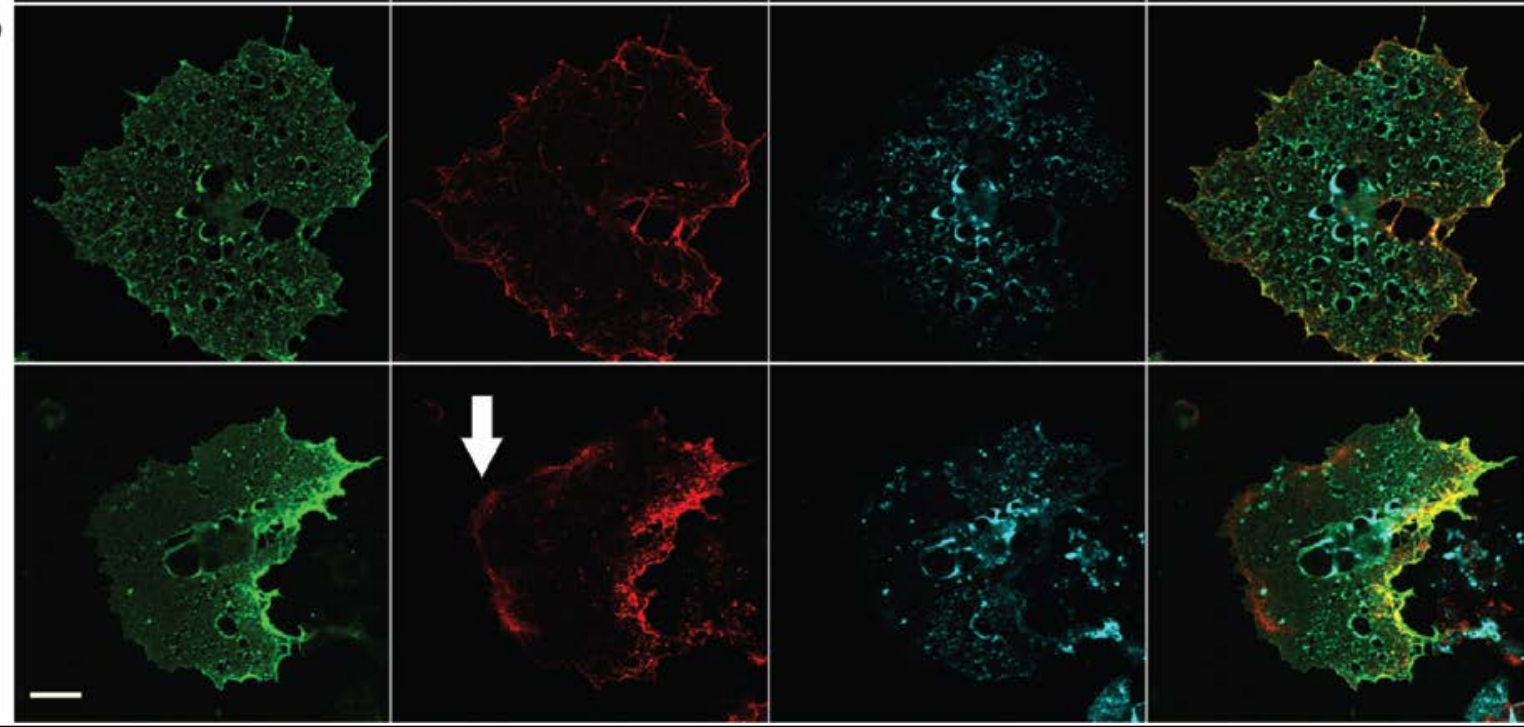

Figure 3.14: Features of the VSV-G distribution on cultured oligodendrocyte

A-E) Oligodendrocyte cell culture infected by VSV for 3 hours and surfaced stained for VSV-G and total staining for F-actin. A) Polarity of the VSV-G trafficking towards the cellular rim that is not in contact with surrounding cells. B-C) The VSV-G labeling intensity is in direct correlation with the amount of F-actin at the rim size; when the F-actin rim is small the VSV-G labeling is not directed towards these areas (arrows). D) The surface staining of VSV-G diffuses freely within the compacted areas of the cultured oligodendrocytes. E) Oligodendrocyte showing a migratory morphology with a polar distribution of the Factin and VSV-G labeling directed opposite to the retractile end. Scale bar $20 \mu \mathrm{m}$. 
Finally, in the rare cases where the oligodendrocytes would show migratory profiles, we could consistently see the VSV-G signal only towards the F-actin rich rim opposite to the retracting end (fig 3.14).

At later time point, 6 or 7 hours post infection, we could observe a punctuate labeling in addition of the diffuse membrane labeling described above. We found this labeling mainly at the cellular rim of the cell, within and on the surface of the oligodendrocytes (fig 3.15). We hypothesized that these punctuate labeling represent the newly synthesized viral particles at their budding site.

Based on these observations we concluded that the insertion of VSV-G upon VSV infection in cultured oligodendrocyte does not occur randomly, but selectively at the most distal region from the cell body - in the cellular rim, from which it would diffuse back into the sheets.

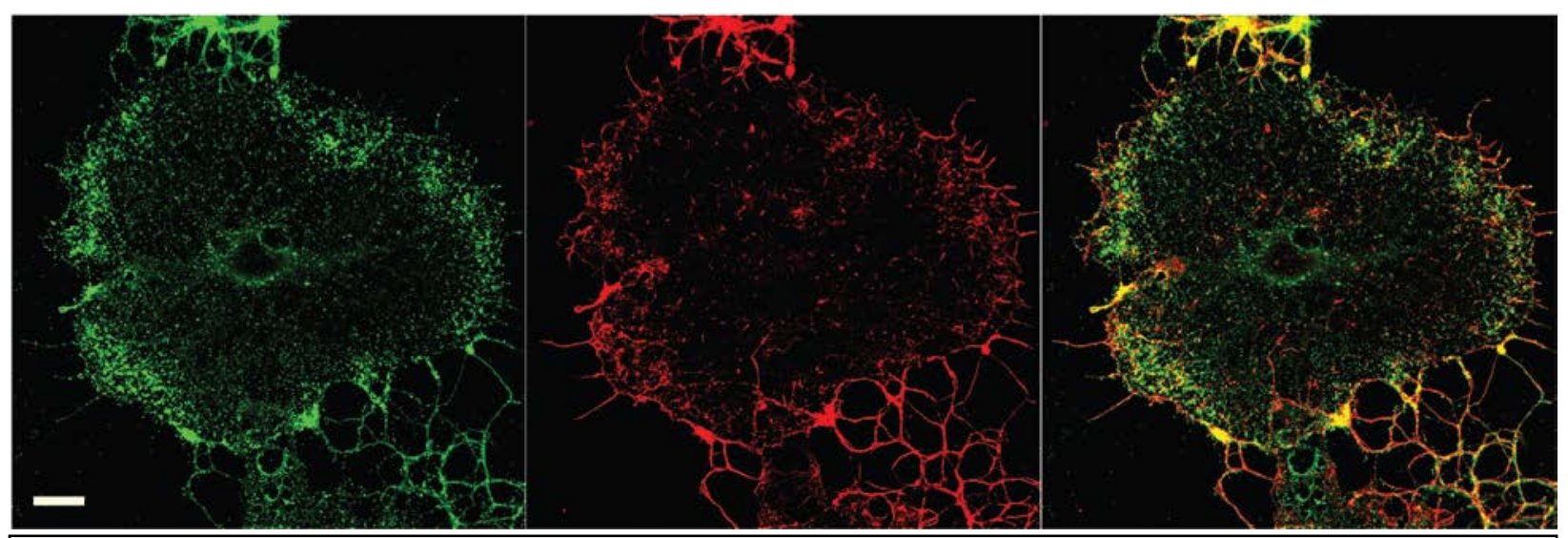

Figure 3.15: VSV particles localization in cell culture.

Oligodendrocyte in culture infected by VSV for 7 hours showing a punctuate labeling mainly located at the cellular rim. Scale bar $20 \mu \mathrm{m}$. 


\subsubsection{Subcellular localization of the VSV-G in growing myelin sheath in vivo.}

Based on the results obtained with the cultured oligodendrocyte we aimed to localize the growth front in vivo by injecting a high titer of VSV $\left(10 \mu \mathrm{l}, 1 \times 10^{11} \mathrm{pfu} / \mathrm{ml}\right)$ in the corpus callosum (CC) of anesthetized mice at P21 (during myelin growth) and P60 (after completion of most of the myelination events) and sacrificing the mice after 1, 3 and 6 hours post infection. The CC is a myelin rich region of the brain easily accessible for injection making it a suitable area of investigation for this experiment. During all the duration of this experiment the mice were kept under anesthesia. The injected areas were extracted and further proceeded for immunoelectron microscopy.

At P21, after one hour, we could not detect any signal in the myelin sheaths neighboring the injection site; only 3 and 6 hours after VSV infection we could detect VSV-G protein in the myelin sheath. When assessing the localization of the labeling of cross sections, within the different compartments of the myelin sheath (the inner tongue, the compacted layers and the outer tongue) we could see that at P21 the VSV-G staining was mainly found in the inner tongue of the infected sheath, but also, to a lesser extent, in the outer tongue and almost none could be found in the compact myelin (fig 3.16). The small intracellular domain of the VSV-G would allow the VSV-G to enter the compact myelin but due to its large extracellular domain the VSV-G is likely to be extruded from the compact myelin. At 6 hours post infection we observed a far denser labeling with a similar distribution pattern of the VSV-G throughout the different compartments of the myelin sheath, but with an even greater enrichment of the VSV$\mathrm{G}$ at the inner tongue (fig 3.16). We complemented this cross-sectional analysis by assessing the localization of the VSV-G along the growing myelin sheath on longitudinal sections and found that the labeling was enriched at the lateral edges of the growing myelin layers (fig 3.16). In addition to the localization of the VSV-G proteins at 6 hours post infection we could visualize viral particles within the myelin sheath almost exclusively in the inner tongue or between the myelin sheath and the axolemma of the underlying axon (fig 3.16). 
A

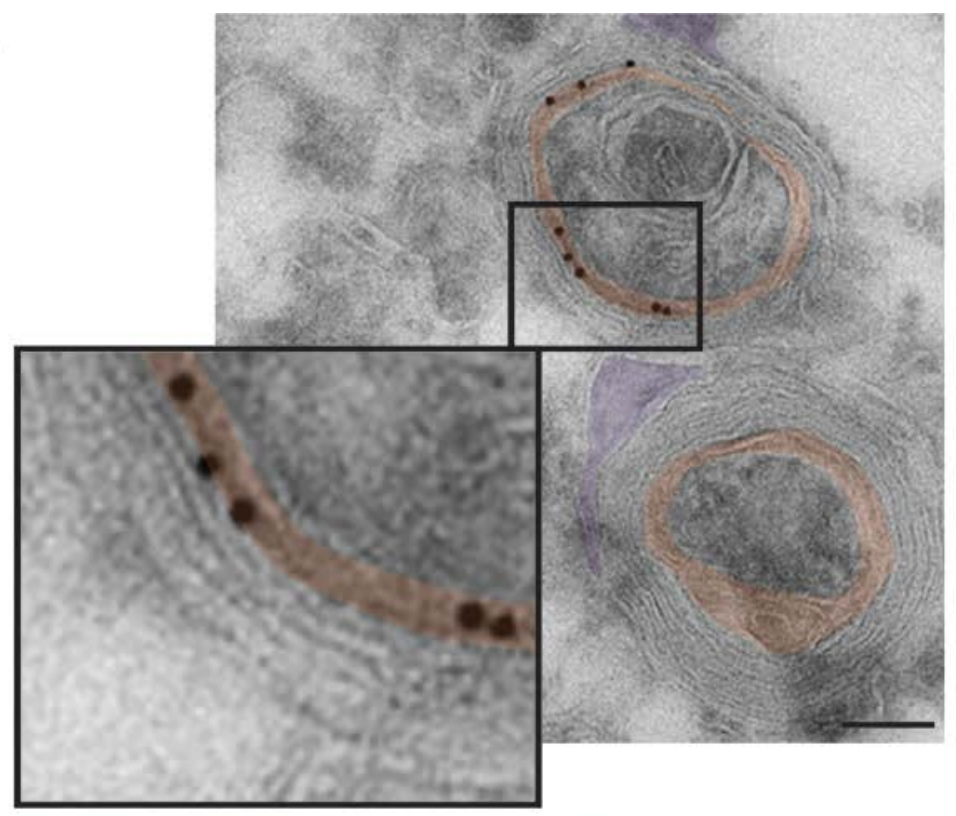

B

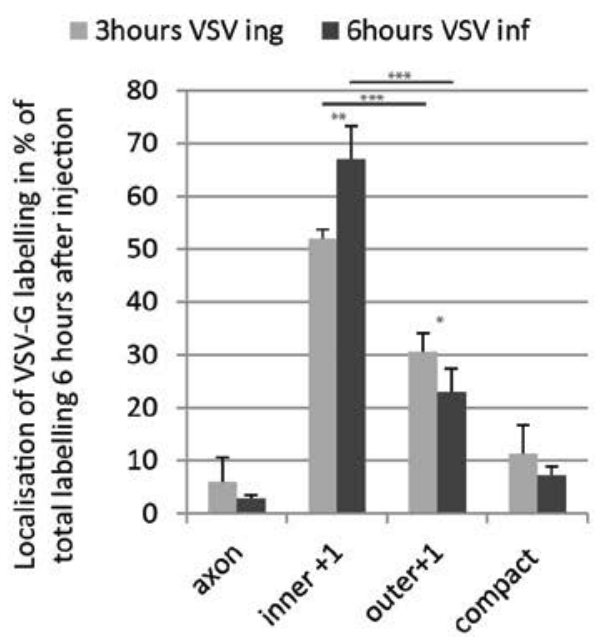

C

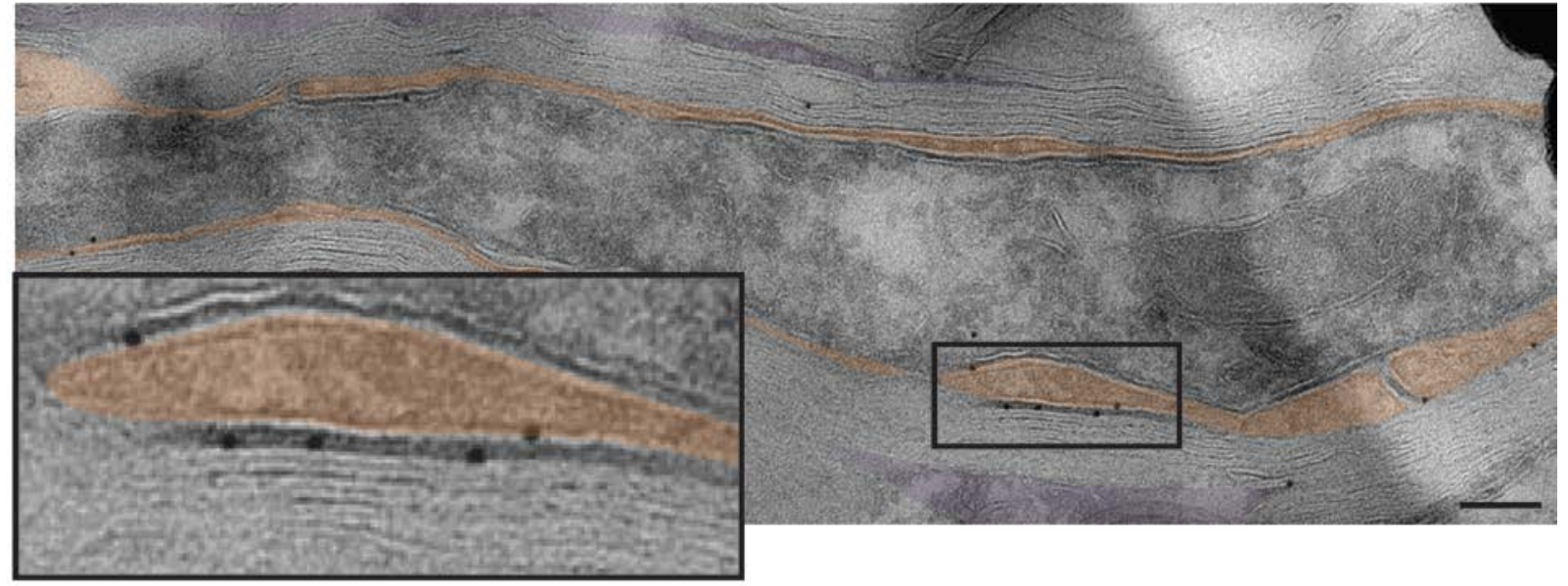

D

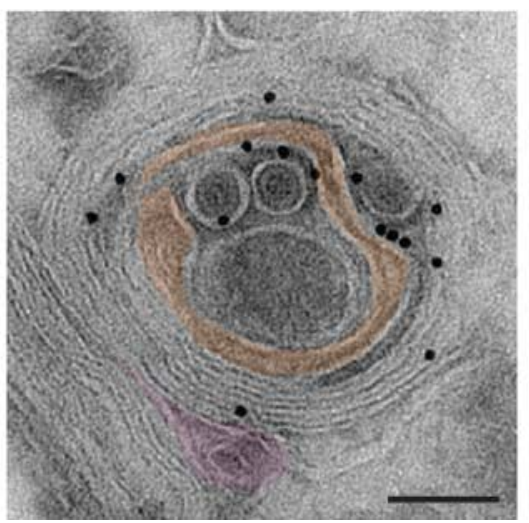

Figure 3.16: Tracking the membrane trafficking using the vesicular stomatitis virus $\mathbf{G}$ protein in vivo during myelination.

A) Sub cellular localization of VSV-G predominantly at the inner tongue in myelinated axon of P21 corpus callosum (CC) infected with a high titer of VSV for 6 hours (orange: inner tongue, purple: outer tongue). B) Quantification of the VSV-G labeling distribution within the different domains of the myelin sheath at P21 after 3 and 6 hours of infection by VSV. C) Longitudinal section of P21 CC infected for 6 hours showing the lateral extremities of the successive inner layers been enriched with VSV-G labeling. D) Localization of VSV particles at the inside of the myelin sheath within the non-compacted areas of the myelin or between the axon and the oligodendrocyte. Bars shows mean \pm SD. Scale bars $200 \mathrm{~nm}$. 
When the VSV was injected in P60 CC, after the completion of myelination, the labeling intensity was reduced as compared to the injection performed at P21. At 6 hours post infection we found the labeling mostly at the outer tongue of the myelin sheath, with little labeling at the inner tongue (fig 3.17). Surprisingly, even at 6 hours post infection almost no viral particles were found in the myelin sheath of the animal investigated. At 3 hours post infection only a very sparse VSV-G labeling was observed at the injection site in the membrane of some myelin sheath. The control mice injected with the balanced salt solution together with $0.3 \%$ of monostral blue alone did not show any labeling after 6 hours congirming the high selectivity of the VSV-G antibody (fig 3.17).

All together these results indicate a polarized delivery and insertion of the newly synthetized material to the inner tongue of the growing myelin sheath as well as a preferred exocytosis of the viral particles from this compartment.
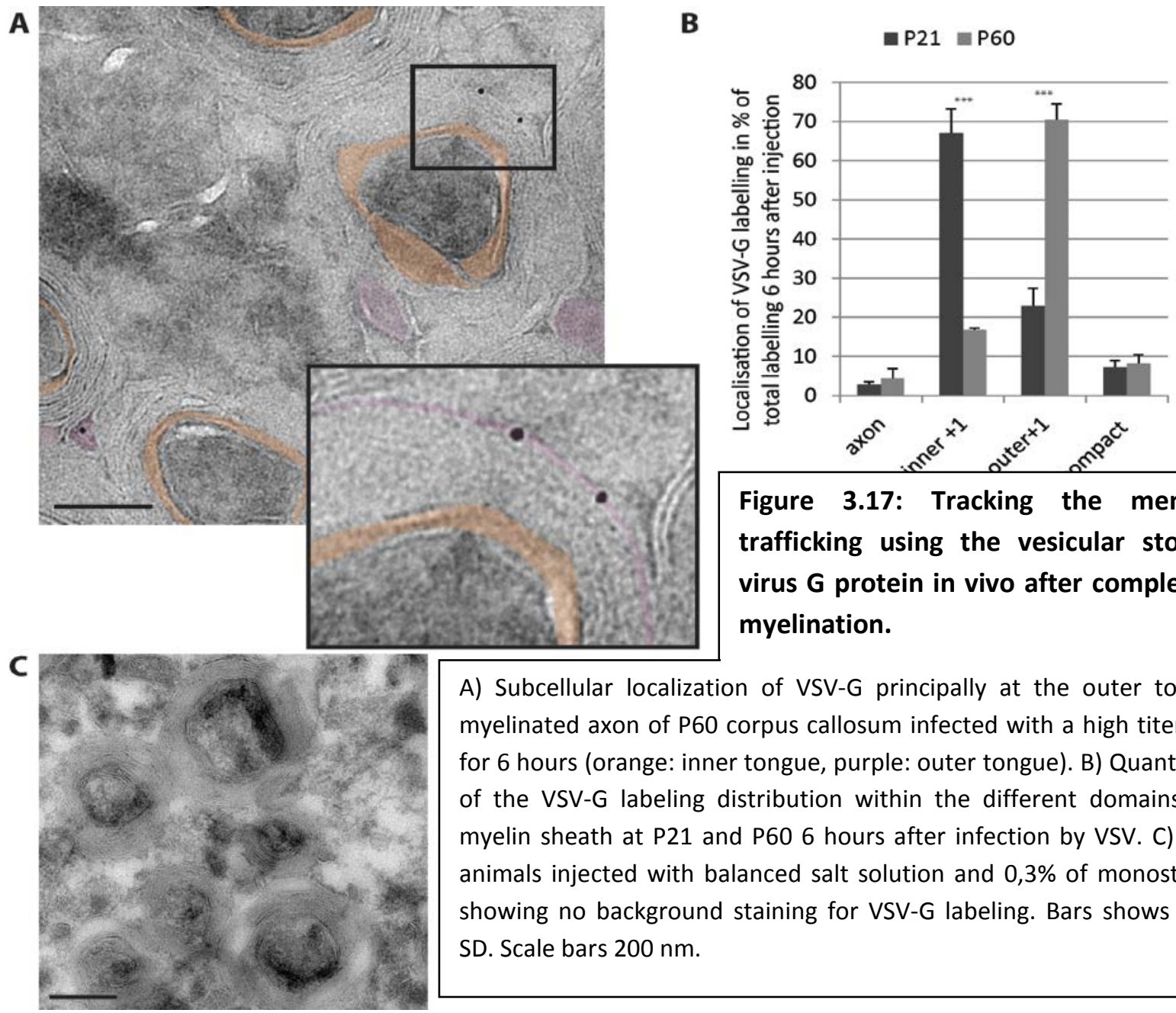

A) Subcellular localization of VSV-G principally at the outer tongue in myelinated axon of P60 corpus callosum infected with a high titer of VSV for 6 hours (orange: inner tongue, purple: outer tongue). B) Quantification of the VSV-G labeling distribution within the different domains of the myelin sheath at P21 and P60 6 hours after infection by VSV. C) Control animals injected with balanced salt solution and $0,3 \%$ of monostral blue showing no background staining for VSV-G labeling. Bars shows mean \pm SD. Scale bars $200 \mathrm{~nm}$. 


\subsection{Characterization of the mechanisms responsible for the cytoplasmic polarity in myelinating oligodendrocyte.}

Our previous results showed that the inner tongue is winding around the axon and also that the newly synthesized material is incorporated at this area in vivo, which correspond to the outer rim of the cultured oligodendrocytes. Based on these findings we wanted to investigate these compartments in more details to understand the mechanisms underlying the polarized plasma membrane trafficking in the developing oligodendrocyte.

\subsubsection{Investigation of the oligodendrocyte inner tongue/outer rim during myelination}

\subsubsection{Cytoskeleton distribution at the oligodendrocyte outer rim/inner tongue}

It is well known that the growth zone in numerous cell types harbor a characteristic cytoplasmic meshwork that is mainly composed of actin and regulatory proteins (Hall, 1998). After identifying the most distal part of the oligodendrocyte processes (inner tongue in vivo and outer rim in vitro) as growth zones using the VSV, we wanted to assess if the growth zone in the myelinating cells presented similar morphological and molecular features.

To investigate the oligodendrocyte growth zone in a simplified system we started our observations on cultured oligodendrocytes. We could confirm the absence of filamentous-actin (f-actin) from the MBP-positive areas of the cells and the heterogeneity in its distribution in the non-compacted areas. Indeed using antibodies against f-actin or phalloidin coupled to rhodahmine we found the f-actin to be enriched at the cellular rim during the growth phase of the oligodendrocyte, between days in vitro (DIV) 2 and DIV 5 (fig 3.18). When investigating this area of the cell in more details we could observe that the shape of the actin rich rim appeared to be different during different phases of the cell growth. Before DIV 3 the f-actin is preferentially forming long fillopodia from the rim of the cell (fig 3.18). After this initial growth/sensing phase the F-actin at the rim is forming lamelipodia with variable thickness according to the maturation stage of the oligodendrocytes in culture (fig 3.18). We then wanted to investigate the correlation between the cell rim thickness and the growth status of the cell. 

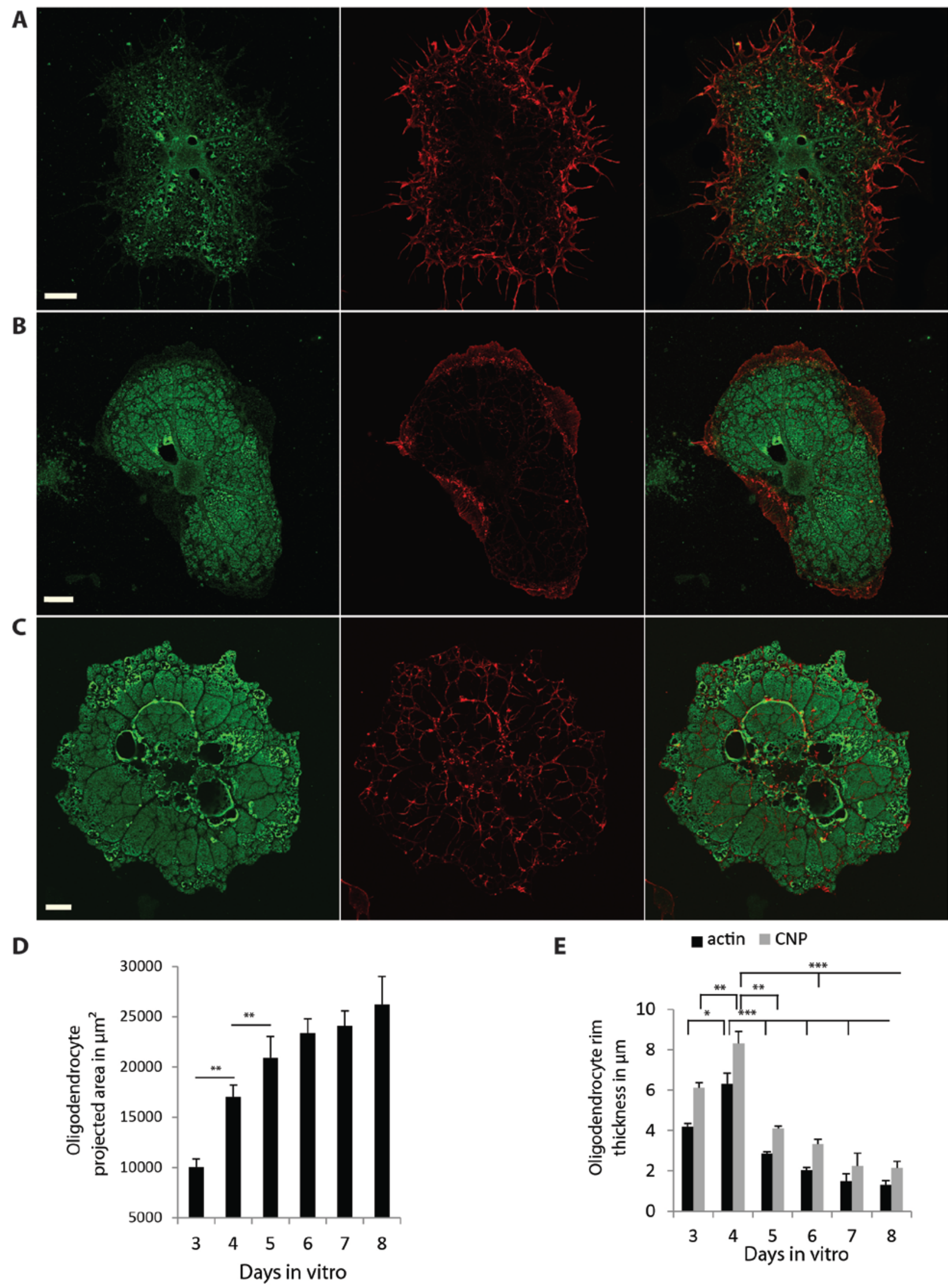

Page | 54 
Figure 3.18: Evolution of the oligodendrocyte morphology upon development in culture.

A-C) Evolution of the F-actin cytoskeleton at the cellular rim. A) Until 3 DIV the F-actin cytoskeleton forms fillopodia from the cell rim and very little MBP can be found in the cells at 3 DIV. B) At 4 DIV the F-actin forms lamelipodia at the rim of the cell and at 6 DIV the cells barely show any F-actin in this compartment (C). D) Quantification of the non-compacted areas (CNP labeling) and the F-actin positive areas at the cellular rim over oligodendrocyte maturation in culture. Bar show mean \pm SD ( $n=3$ independent cell cultures, 30 cells per culture, 4 measures per cell). E) Average projected area of the oligodendrocyte in culture during development. Bar show mean \pm SD ( $n=3$ independent cell cultures, 30 cells per culture). Scale bars $20 \mu \mathrm{m}$.

We measured the projected area of the cell, the non-compacted area at the cellular rim using the antibodies against CNP, as well as the size of the F-actin zone (lamelipodia thickness) during the oligodendrocyte maturation in culture. We could see that between DIV 3 and 5, the areas of the cells increased more than 2 folds, marking the peak of the growth in culture. From DIV 5 to 8 the cells would not get significantly bigger. Interestingly, the cellular F-actin-rich rims were significantly bigger at DIV3 and 4 than when the cell slows its growth between DIV 5 and 6 (fig 3.18). By DIV 7 and 8 the size of the cellular rim was greatly reduced and almost not CNP or Factin signal could be found (fig 3.18).

Based on the in vitro data we next wanted to assess the F-actin distribution in vivo. Using $50 \mathrm{~nm}$ Tokuyasu sections we intended to visualize the F-actin cytoskeleton with EM to assess the subcellular distribution of the F-actin with a greater resolution. However, due to the size of the filaments, the relatively low efficiency of the Tokuyasu labeling technique and the poor specificity of the antibodies we couldn't reliably localize the F-actin by EM. To overcome these technical limitations we used 300nm thick Tokuyasu sections of P4 and P60 spinal cord thoracic cross section, during the myelin growth phase (P4) and when the myelination is finished (P60) and processed them for light microscopy analysis. We focused our analysis on the dorsal column where the axons and the myelin sheath are the biggest, allowing a more accurate localization of the staining by light microscopy. With such a thin cross section of the spinal cord we could observe F-actin-rich patches with phalloidin-rhodamin staining in over 50 percent of the P4 myelin sheath (fig 3.19). The actin co-localized with the myelin associated glycoprotein (MAG) staining (Trapp et al., 1989). Indeed, the f-actin labeling was consistently found inside the myelin sheath labeled by MBP staining (fig 3.19). When investigating the spinal cord after 
myelination was completed, at $\mathrm{P} 60$, we found that the f-actin labeling was almost notdetectable, supporting our previous cell culture data where we found a clear correlation between growth phase and f-actin labeling (fig 19).

Based on these findings we generated a colored electron micrograph with the hypothetical localization of the f-actin in comparison to the MAG and MBP positive areas (fig 19).

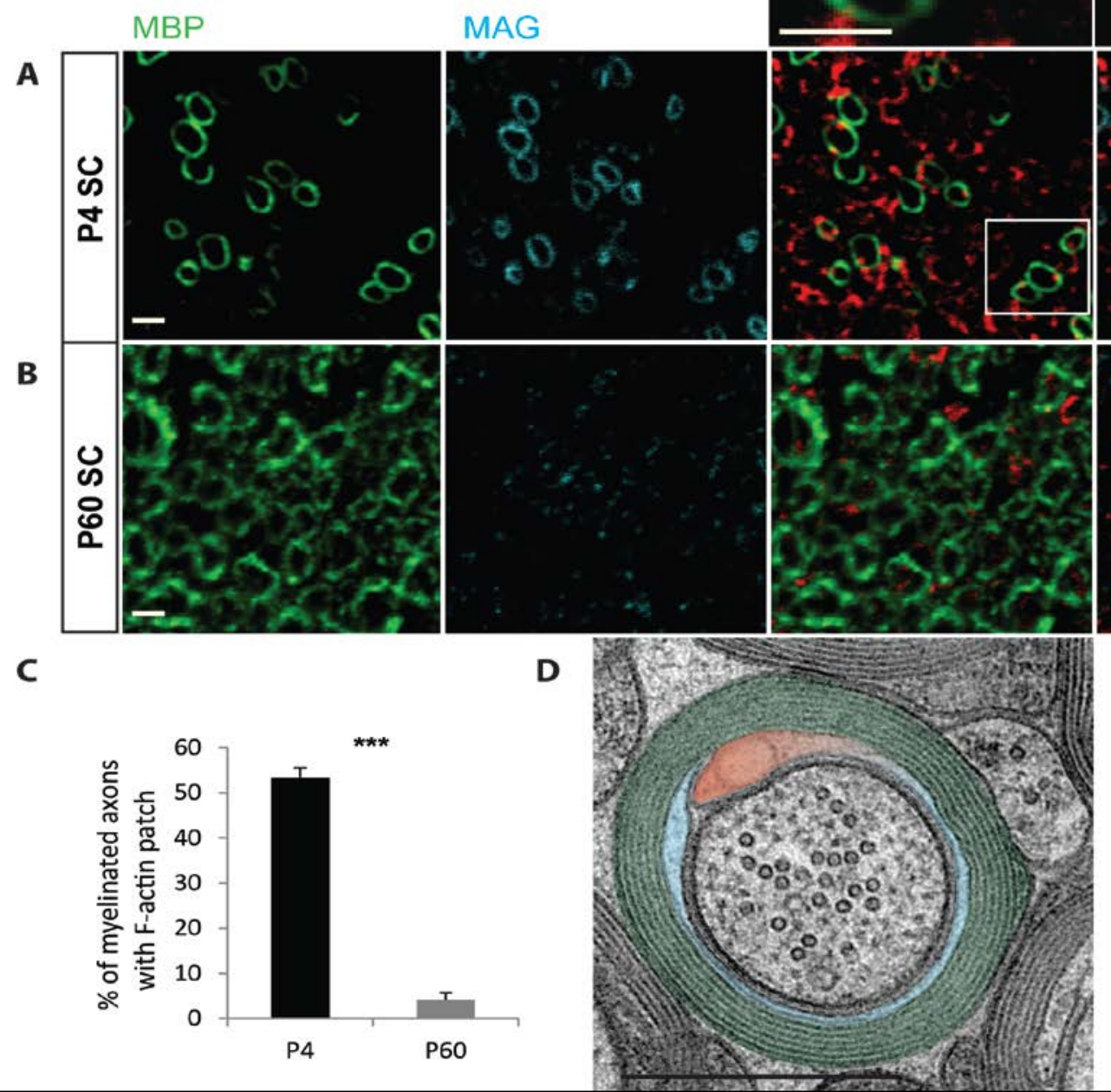

Figure 3.19: Localization of the f-actin cytoskeleton in vivo.

A, B) Semi-thin cyo-sections ( $400 \mathrm{~nm}$ ) of P4 (A) and P60 (B) spinal cord labeled for MBP, MAG and F-actin. At P4 a large amount of the myelinated sheaths show a patch of F-actin located inside the MBP positive area, but colocalizing with the MAG positive area. At P60 almost no MAG or $\mathrm{f}$-actin could be found in the myelin sheath. C) Quantification of the myelin sheath presenting a f-actin patch within the ring-like MBP staining at P4 and P60. Bars show mean \pm SD ( $n=3$ animal, 100 axons for P4 and 1000 axons for P60). Scale bar $20 \mu \mathrm{m}$.D) Putative distribution of the MBP (green), MAG (blue) and F-actin (red) proteins in an electron micrograph of myelinated axon. Scale bar $500 \mathrm{~nm}$. 


\subsubsection{Morphological evolution of the inner tongue in vivo during myelin growth}

After localizing the F-actin at the growth zone we wanted to investigate the morphological remodeling of the inner tongue during the growth phase of the myelin sheath in vivo. To do so, we investigated the inner tongue and outer tongue size as well as the myelin thickness on the growing myelin sheath in the optic nerve between P10 and P60. We found that at P10 the area of the inner tongue was in average almost as large as the area of the axon (around 90 percent), whereas the area of the outer tongue covered only around 20 percent of the area of the axon (fig 3.20). With the maturation of the myelin sheaths we could see a sharp reduction of the inner tongue size. At P60 the ratio of the inner tongue area as compared to the axon area was less than 20 percent. For the outer tongue however, we could not detect a significant reduction between the 4 ages assessed (P10, P14, P21 and P60) (fig 3.20). These data would correlate with our findings in the outer rim of the growing oligodendrocyte in culture.

Based on these observations in vitro and in vivo we could show that the F-actin amount and the cytoplasmic compartment size at the inner tongue or outer rim would directly correlate with the growth behavior of the oligodendrocyte. 

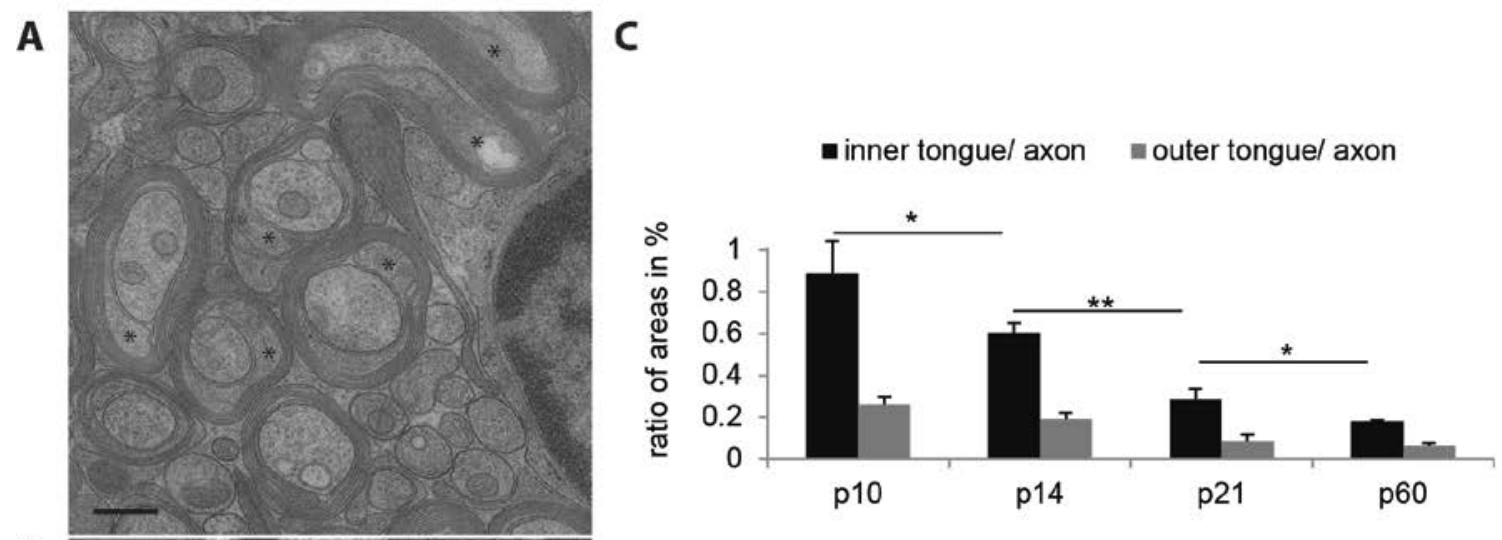

B
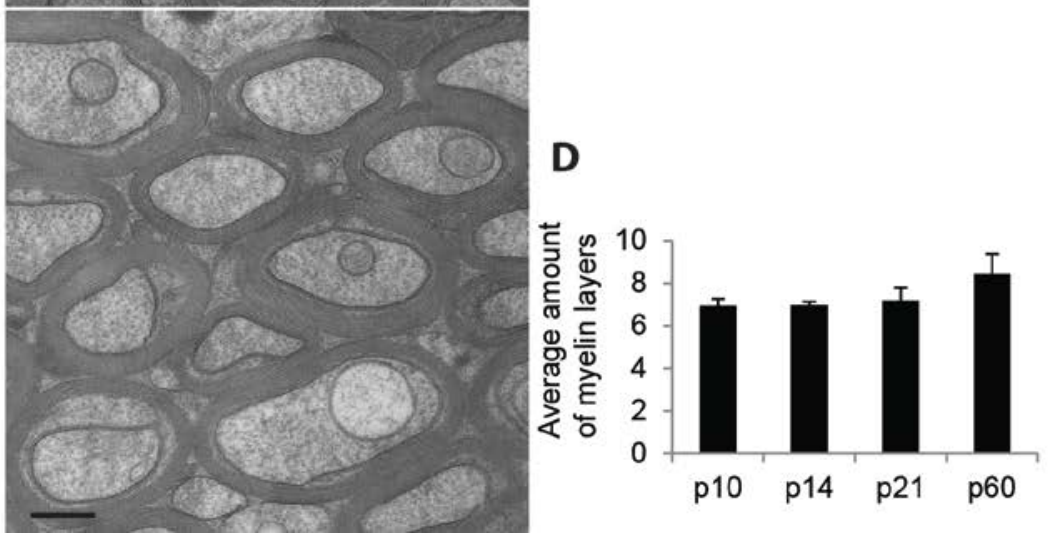

E

Figure 3.20: Evolution of the morphology of the non-compacted areas of the myelin sheath upon development in vivo.

A, B) Electron micrographs of high pressure frozen optic nerves at P14 (A) and P60 (B). The inner tongue compartments of the sheets appear larger at P14 (stars) than at P60. C) Quantification of the inner and outer tongues areas reported to the axon size between P10 and P60 showing a massive reduction of the inner tongue size during development. D) Average amount of myelin layers of the quantified axons during development from P10 to P60. E) Quantification of the axon area during development from P10 to P60. Bar show mean $\pm S D$ ( $n=3$ animal, 50 axons per animal). Scale bar $500 \mathrm{~nm}$.

\subsubsection{Oligodendrocytic cytoplasm polarization during the myelin growth}

Using VSV we could identify that inner tongue is the site of incorporation of the newly synthesized material to the growing myelin sheath. Furthermore, we could correlate the growth of the leading edge with an active F-actin cytoskeleton and a larger cytoplasmic zone. We next wanted to investigate the molecular mechanisms underlying these morphological changes and the polarized material delivery within the growing myelin sheath. 


\subsubsection{The PI3K/AKT/mTOR signaling pathway is enriched at the leading edge of the oligodendrocyte}

The polarized distribution and the shape of the f-actin during the growth phase of the oligodendrocyte as well as the morphology of these growth zones lead us to investigate the localization of some major known growth regulators that are involved in the cytoskeleton remodeling. A central pathway involved in the myelin growth and cytoskeleton remodeling during cell growth and migration is the PI3K/AKT/mTOR signaling pathway. The localization of the proteins or lipids involved in the oligodendrocyte cell polarity was complicated due to the fact that it is impossible to differentiate the staining of a proteins or lipids that are exerting their function or are been transported toward the zone where they will perform their action. For this reason we investigated the localization of Phospho-Akt protein, a downstream product of the PI3K that is present in its phosphorylated form only when activated at the target site. In reason of its role in polarize growth of cell, Phospho-Akt appeared to be as ideal target for the localization of the oligodendrocyte growth zone (Fayard et al., 2010). We could show that at DIV 3 and 4 on oligodendrocyte culture the Phospho-Akt 473 (P-Sec-473) labeling was heavily polarized towards the cellular rim (fig 3.21). Indeed the signal would rapidly disappear in the cytoplasmic rich processes connecting the outer rim and the cell body where only a fainted signal could be observed. In presence of cortical neurons the oligodendrocytes in culture send several processes to contact the neighboring axons and subsequently initiate wrapping (ref). At an early stage of this co-culture we could visualize an intense phopho-Akt staining at the leading edge of the multiple oligodendrocytic processes extending towards the neighboring axons (fig 3.21).

In addition to Phospho-Akt we investigated the distribution of PIP3 and PI3K in DIV4 oligodendrocytes. Since available PI $(3,4,5)$ and PI3K antibodies produced high background in immunofluorescence, we visualized these molecules by using the PI(3,4,5) P3-PH-GFP sensor and by over-expressing the GFP-tagged PI3K construct in cultured oligodendrocytes. In both experiments we could confirm a gradient towards the cellular rim for both PIP3 and PI3K, but the enrichment of the signal gradient was less pronounced as compared to Phopho-Akt (P-Ser473) (fig 3.21). 
A
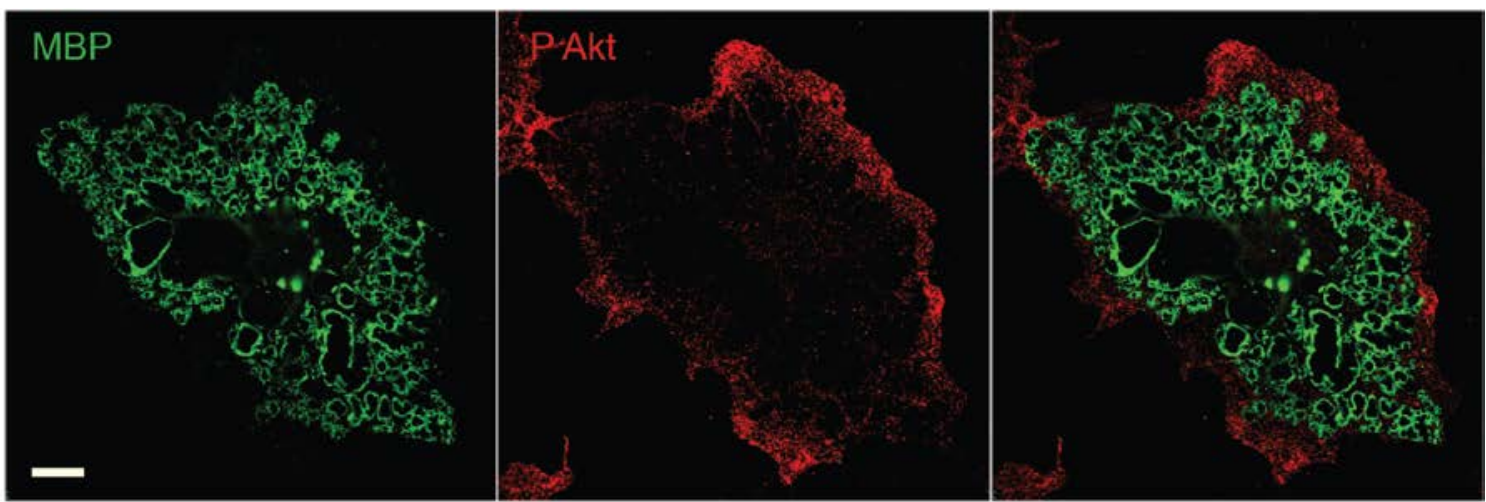

B

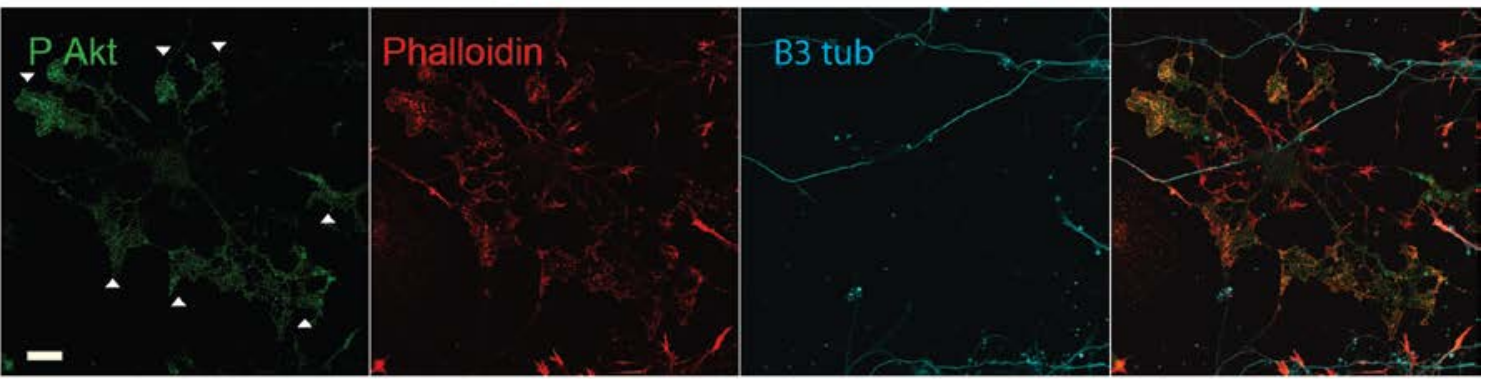

C

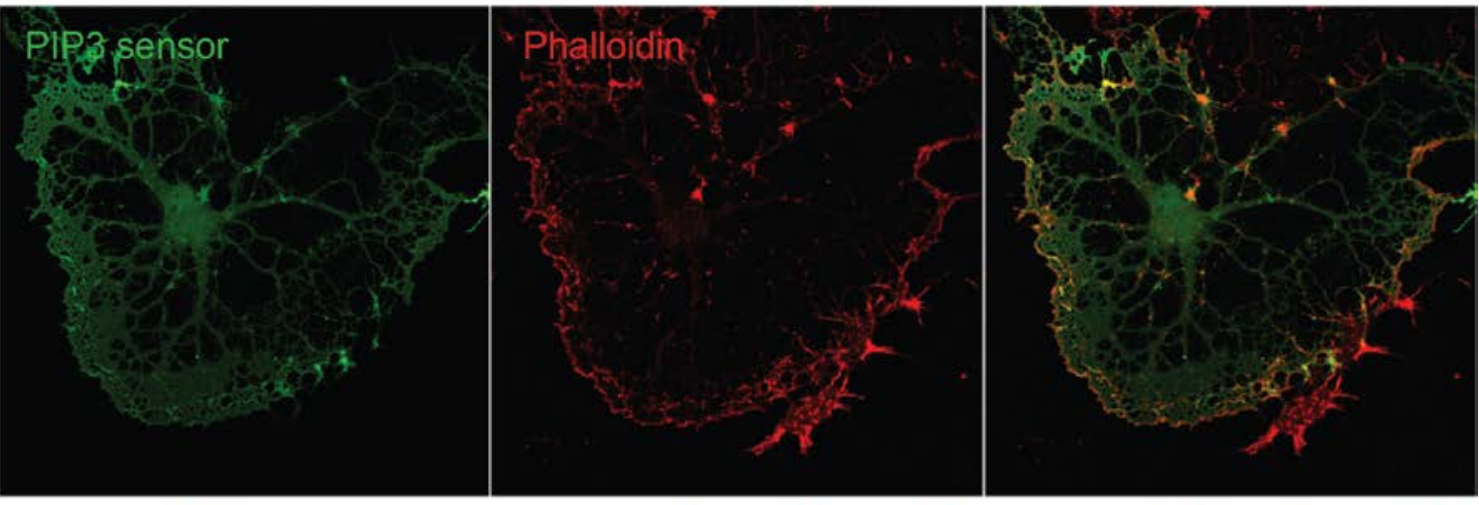

D

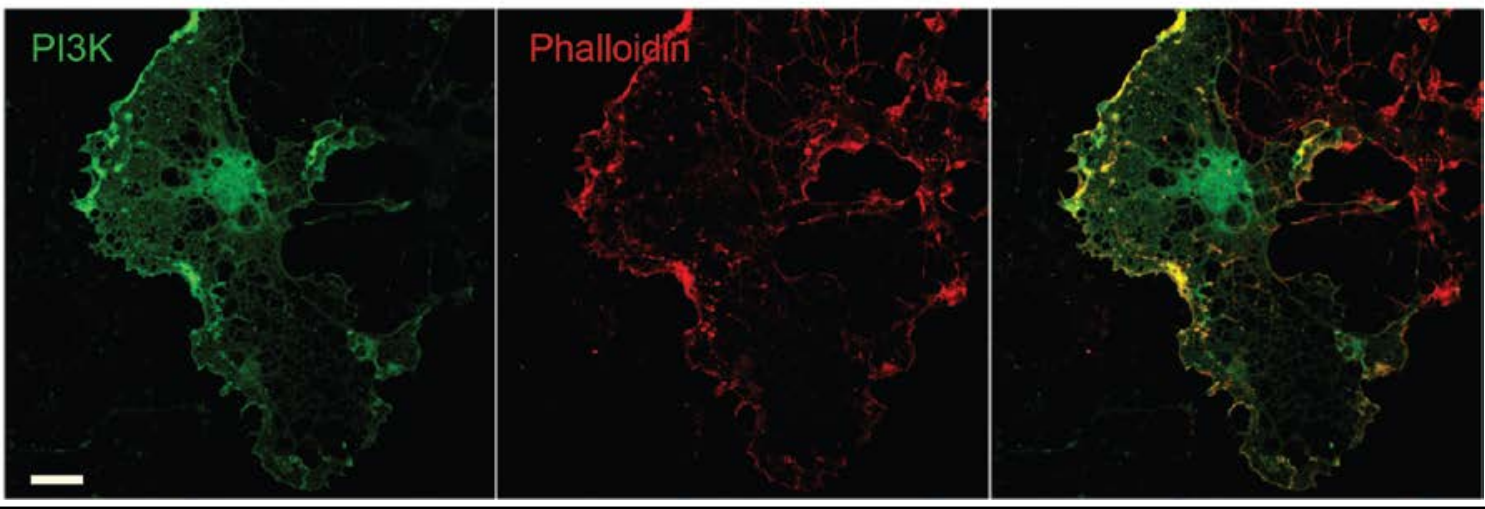

Figure 3.21: Cytoplasmic polarization of the oligodendrocyte during the growth.

A, B) Polarized signal for the phopho-Akt within the cytoplasmic compartments of the growing oligodendrocyte: at the cellular rim (A), or at the tip of the oligodendrocytic processes sent towards axons in a cortical co-culture (arrows) (B). C, D) After transfection of the oligodendrocytes with GFP-C1$\mathrm{PH}$ and PI3KCA-WT plasmids a similar gradients could be observed at the cellular rim indicating the respective polarization of the PIP3 and PI3Kinase molecules. Scale bar $20 \mu \mathrm{m}$ 


\subsubsection{Regulation of the oligodendrocyte growth by PI3K/AKT/mTOR signaling pathway}

\subsection{In vitro}

After localizing main components of the PI3K pathway at the oligodendrocyte growth zone we wanted to assess the effects of the PI3K pathway on the cell growth by activating or inhibiting the pathway at different levels. We applied in vitro inhibitors onto oligodendrocytes in culture for several enzymes of the PI3K pathway. Inhibition of the receptor tyrosine kinase (Genistein), PI3K (Ly294002), Akt kinase (Akt 1/2 kinase inhibitor) and mTOR complex (Rapamycin) would lead to the inhibition of the pathway when inhibiting PTEN (VO-OHpic) would enhance the Phospho-Akt signaling (fig 3.22). The inhibitors were applied for $24 \mathrm{~h}$ from DIV3 to DIV4 on a cell culture with low cellular density (20K cells per $13 \mathrm{~mm}$ coverslip) to analyze the effects and morphology of isolated cells. Using a standard dose of these inhibitors (see material and methods) we measured the f-actin and CNPase labeling size at the rim of the cells. We could observe a significant reduction in the F-actin lamelipodia thickness and non-compacted area (CNPase labeling) at the cellular rim when PIP3, Phospho-Akt or mTOR levels were reduced by these small molecules (fig 3.22). Conversely, by raising the levels of PIP3 in the cell via inhibition of PTEN, the f-actin-rich rim grew in size suggesting that the Phospho-Akt pathway is a main regulator of the oligodendrocyte growth zone (fig 3.22).

\subsection{In vivo}

Based on these findings we investigated the effects of the PTEN inhibition in vivo using

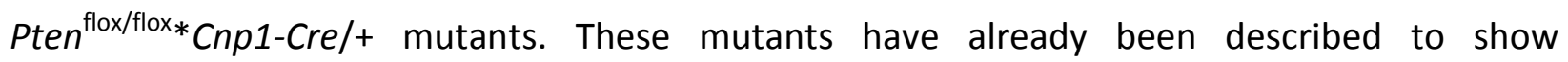
abnormally thick myelin sheath as well as pathological features of redundant myelin (Goebbels et al., 2010). We investigated the optic nerves of the PTEN mutant at P23, a late myelin growth phase, and at P60, a young adult stage.

We could confirm the published data showing a significant enhancement of the myelin thickness at the two time points and the first patterns of redundant myelin reflecting an abnormal growth of the myelin sheath in these mutants (fig 3.23). 


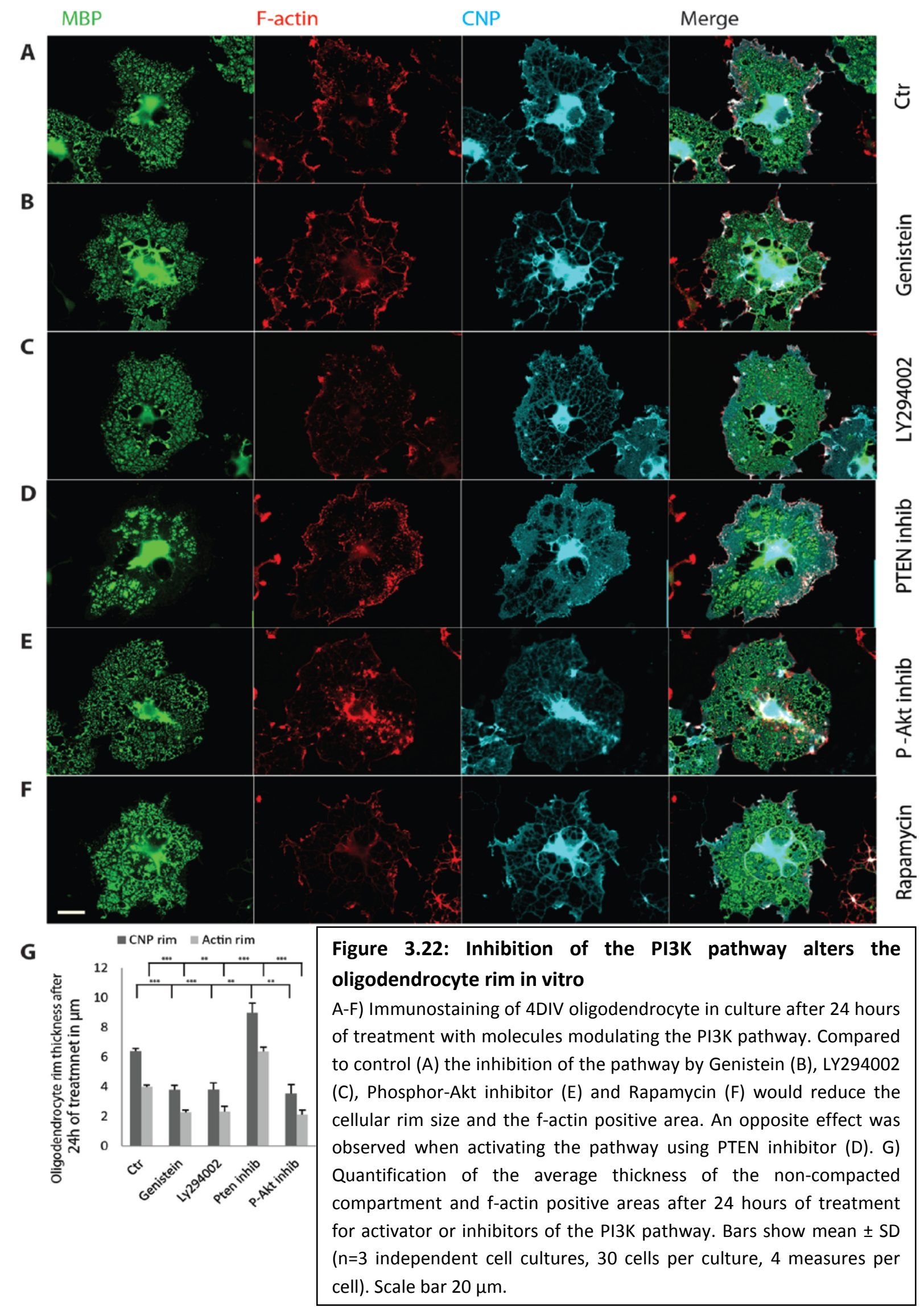


Based on our previous experiments on developing myelin sheath in optic nerves we then assessed the inner and outer tongue size of the myelin sheath in Pten ${ }^{\text {flox/flox* }}$ Cnp1-Cre/+ mutants optic nerves and found that the inner tongue was significantly larger in the PTEN mutant at P23 and P60. However, the outer tongue showed no increase in size (fig 3.23). The ratio of the inner tongue area compared to the axon area at P60 in the Pten ${ }^{\text {flox/flox* }} \mathrm{Cnp1-Cre/+}$ mutants was comparable to the ratios observed in the P10 wild type animals.

Our in vivo data confirmed the results observed in the cell culture and strengthened our hypothesis, where the size of the inner tongue as well as its F-actin content correlate with the growth state of the oligodendrocyte and would directly be dependent on the PIP3 levels in the cells.

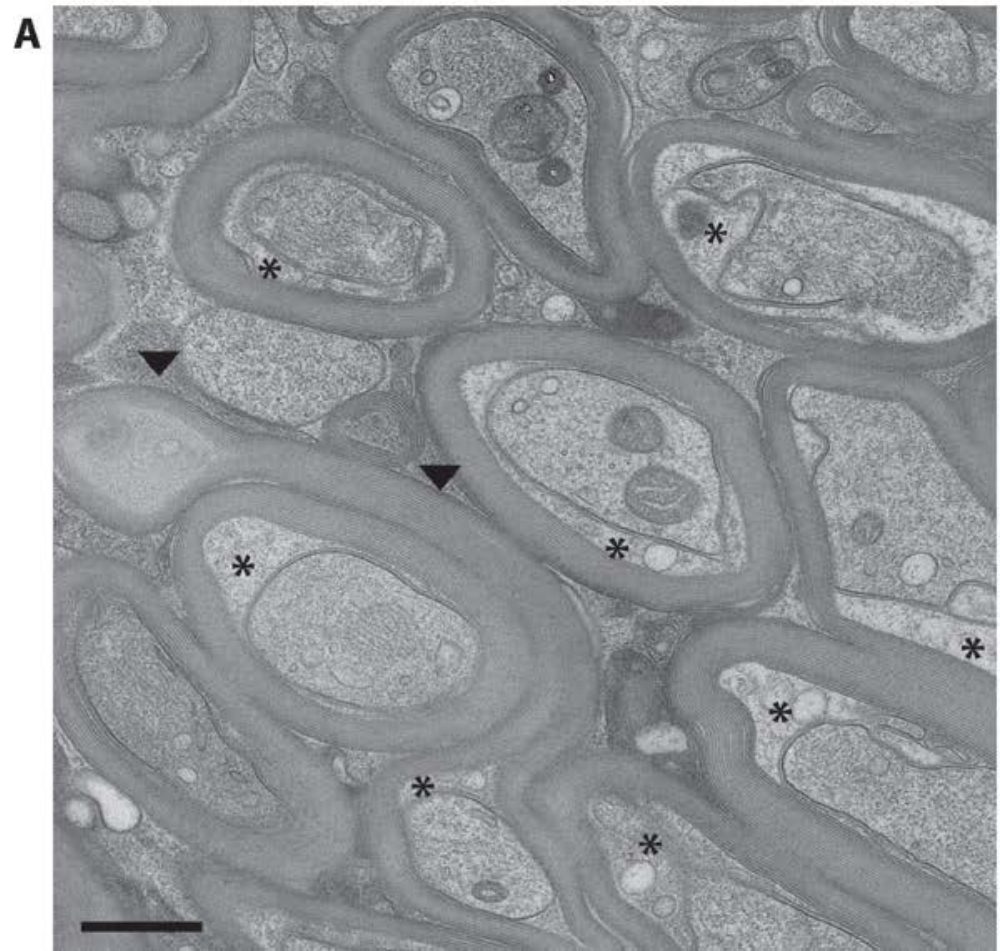

B

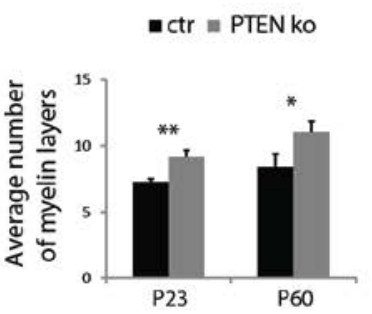

C

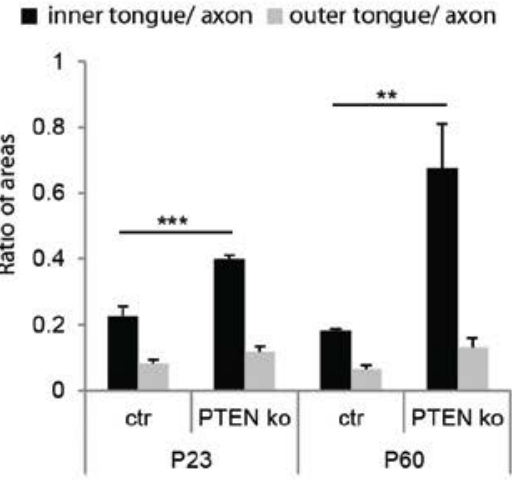

Figure 3.23: Myelin morphology after PTEN inactivation

A) Electron micrograph of P23 PTEN mutant optic nerve showing the myelin sheath with enlarged inner tongue (stars) and outfoldings of compacted layers (black arrows). B) Average thickness of the optic nerve myelin sheath at P23 and P60 in the PTEN mutants compared to control. C) Quantification of the inner and outer tongues areas reported to the axon size between P23 and P60 in PTEN and control animals showing a large increase of the inner tongue size upon PTEN knockout. Bars show mean \pm SD ( $n=3$ animal, 50 axons per animal). Scale bar $500 \mathrm{~nm}$ 


\subsubsection{Artificial polarization of the oligodendrocyte cell trafficking by electrical stimulation}

We went one step further and wanted to investigate possible factors that would influence the cytoplasmic polarization in the oligodendrocyte. In the central nervous system little is known about the cues that trigger the polarization/attraction of the oligodendrocyte towards the axons and initiate the myelination. We made use of an established technique used to artificially polarize cells and trigger their motility and growth during wound healing assay by applying a weak electrical stimulation onto the cultured oligodendrocytes (Tai et al., 2009). Taking advantage of the primary oligodendrocyte culture qualities we seeded the oligodendrocytes on a commercial setup designed for chemotaxi (micro slide from Ibidi, fig 3.24) and applied an electrical current to the cells when they would reach DIV 4. The cells were subjected to an electric current of $1 \mathrm{~V} / \mathrm{cm}$ sustained for 2.5 to 3 hours at $37 \mathrm{C}$ and were fixed directly after the incubation time. Electrical stimulation of oligodendrocyte resulted in a polarization of the Phospho-Akt (P-Ser-473) labeling towards the rim directed to the cathode in over 60 percent of the cells (white arrow heads in fig 3.24) as well as an enrichment of the f-actin labeling in over 80 percent of the cells (fig 3.24). Having established a system to polarize cellular growth zone, we asked whether membrane trafficking could be polarized as well. For this purpose, we infected the cells prior to electrical stimulation with VSV $\left(5 \times 10^{5} \mathrm{pfu} / \mathrm{ml}\right)$ for 15 minutes, washed away the viral particles not bound to the cells and applied the electrical field to the oligodendrocytes. We could visualize a polarized VSV-G signal towards the cathode in around 60 percent of the cells (white arrow heads, fig 3.24).

In this section we could show that the inner tongue in vivo and the corresponding outer rim in vitro harbor similar morphological and molecular features during the growth phase. We found that the plasma membrane trafficking machinery within the myelin sheath is directed towards the inner tongue of the sheath by using the VSV as a reporter. Furthermore, we observed that a gradient of molecules belonging to the PI3K pathway as well as the f-actin cytoskeleton would be enriched at the growth zone during oligodendrocyte development. Finally, we could observe an artificial polarization of the PI3K signaling molecules towards the cathode upon weak 
electrical stimulation and found that the plasma membrane trafficking would follow the polarized growth zone.
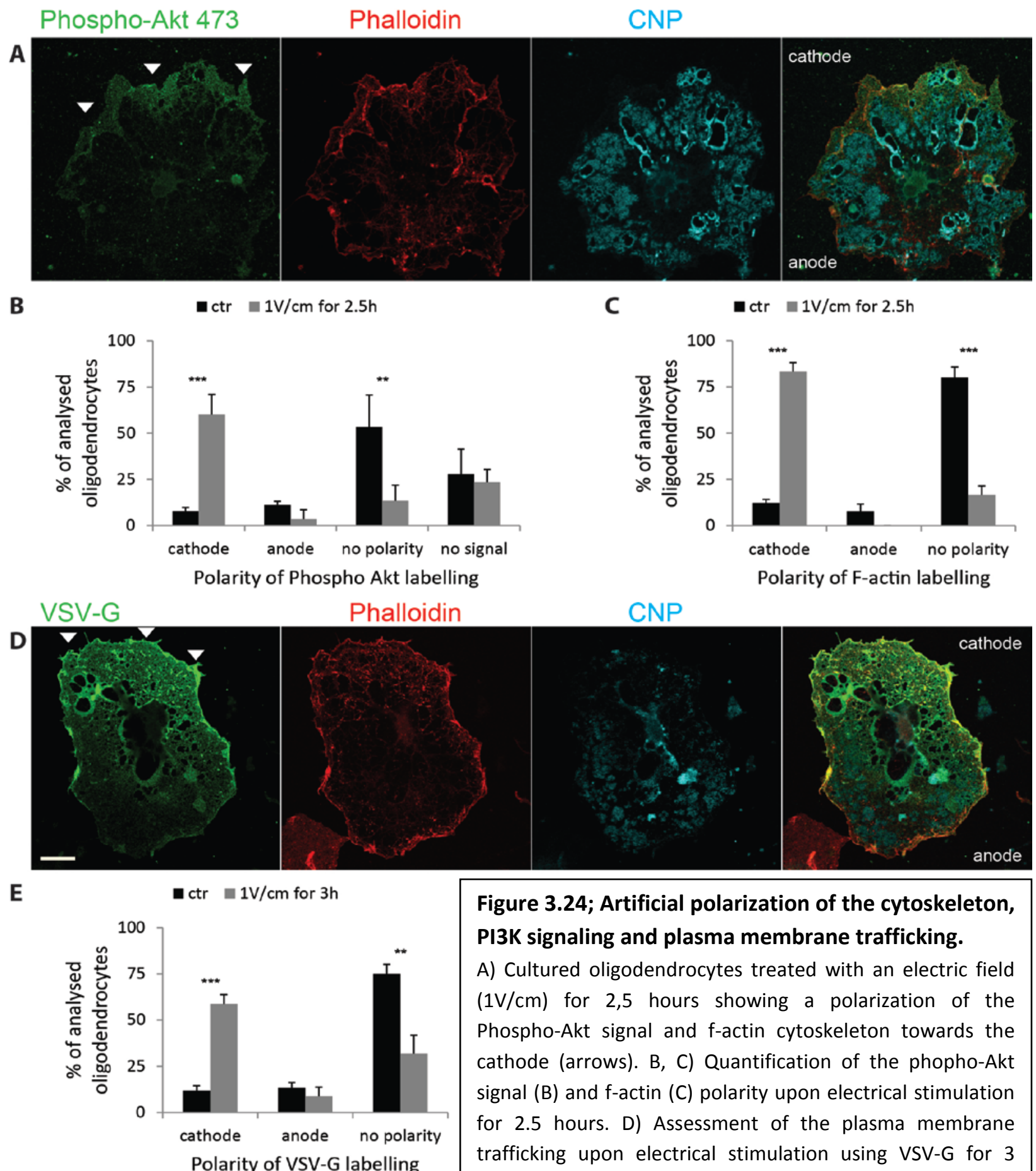

CNP

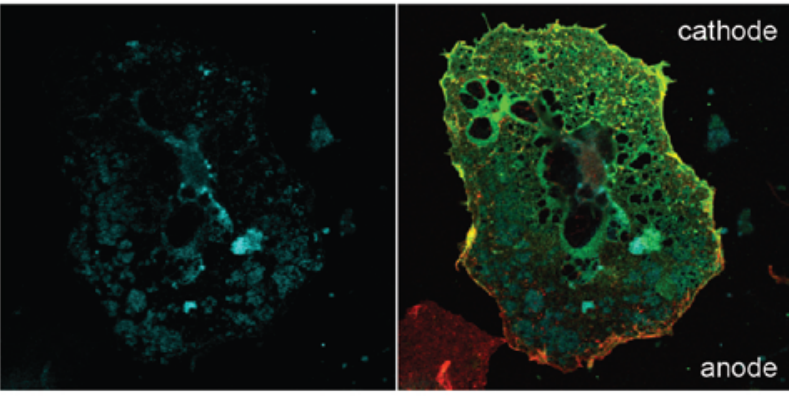

Figure 3.24; Artificial polarization of the cytoskeleton, PI3K signaling and plasma membrane trafficking.

A) Cultured oligodendrocytes treated with an electric field $(1 \mathrm{~V} / \mathrm{cm})$ for 2,5 hours showing a polarization of the Phospho-Akt signal and $f$-actin cytoskeleton towards the cathode (arrows). B, C) Quantification of the phopho-Akt signal (B) and f-actin (C) polarity upon electrical stimulation for 2.5 hours. D) Assessment of the plasma membrane trafficking upon electrical stimulation using VSV-G for 3 hours. The transport of material is largely polarized towards the cathode (arrows). E) Quantification of the VSV-G signal upon electrical stimulation for 3 hours. Bar show mean \pm SD ( 3 independents culture, 30 cells per culture). Scale bar $20 \mu \mathrm{m}$. 


\subsection{Myelin growth associated features in vivo}

Based on the morphology of the growing myelin sheath, the molecular polarity within the oligodendrocytes and the localization of the plasma trafficking reporter (VSV-G) we could show that the inner tongue is the area where the newly synthesized material is directed and where it is incorporated into the growing myelin sheath. We next wanted to investigate the transport modalities of the newly synthesized material towards the inner tongue and also the effects of the cytoplasmic compaction by MBP during the myelin growth and maturation process.

\subsubsection{Cytoplasmic channels provide a shortcut towards the leading edge}

During the intense growth phase of the myelin in the optic nerve we could observe a large amount of growing myelin sheaths showing cytoplasmic inclusions within the compacted myelin. These inclusions, further referred to as cytoplasmic channels, are reminiscent to the Schmidt-Lanterman incisures described in the thick myelin sheath of the peripheral nervous system (Small et al., 1987). These structures, abundant in the PNS, have only rarely been described in the CNS (Hirano and Dembitzer, 1967); (Bunge, 1968). To investigate the morphology of these cytoplasmic channels during development in the optic nerve, we used high pressure freezing immobilization to avoid any artifacts due to chemical fixation and to obtain a close to native preservation of this nervous tissues. When assessing the occurrence of the cytoplasmic channels within the compacted myelin sheath during the optic nerve development, we found that $25,4 \pm 5,4$ percent of the myelinated axons presented cytoplasmic channels at P10 and that very rapidly during maturation these channels would close and only $1,6 \pm 0,9$ percent would remain at P60 (fig 3.25). We could also observe that the size of the cytoplasmic channels would decrease with time as well (fig 3.25). In most of the investigated cytoplasmic channels at P10 and P14 we observed see microtubules in TEM cross sections cut perpendicularly, indicating that these channels are running along the axon (fig 3.25). However when the myelin sheath were imaged longitudinally we also could see that the outer tongue and inner tongue were linked by the cytoplasmic channels within a short distance recalling the morphology of the Schmidt-Lanterman incisures described in the PNS (fig 3.25). 

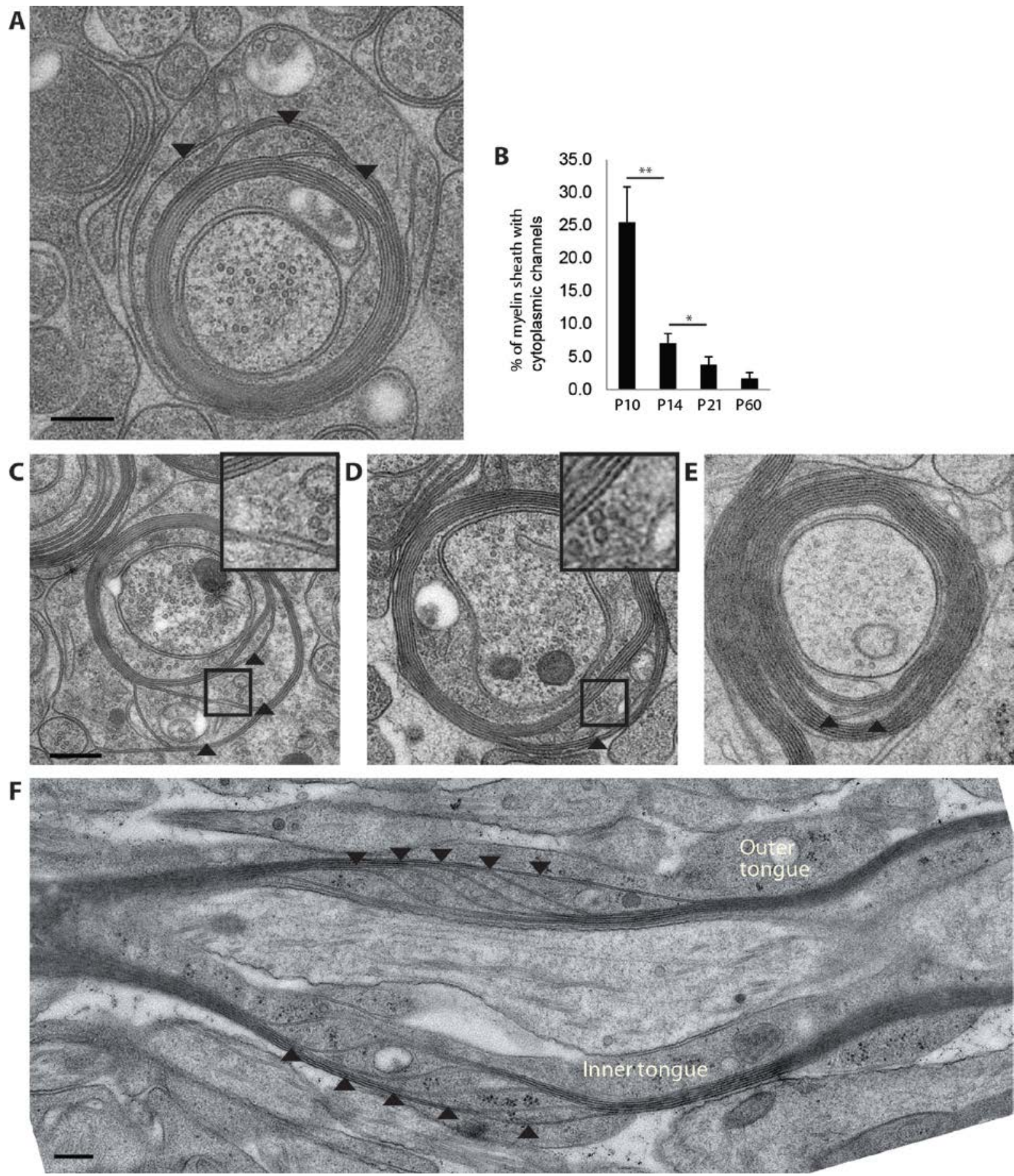

Figure 3.25: Cytoplasmic channels within compacted CNS myelin.

A) Electron micrograph of P10 HPF optic nerve showing cytoplasmic compartments within the compact myelin (black). B) Quantification of the myelin sheath presenting cytoplasmic channels during development at P10, P14, P21 and P60 (3-5 animals, 200 axons per animal. C) Cross section of P10, P14 and P21 myelin sheets in which microtubules and vesicles can be observed within the cytoplasmic channels. The amount of cytoplasm within these channels progressively reduces during the development. Bar show mean $\pm S D(n=3$ animal, 200 axons per animal). Scale bar $200 \mathrm{~nm}$. 
These differences of morphology led us to ask the question of the spatial organization of these channels along the myelinating sheath, and to fully understand their conformation we used the FIBSEM to model in three dimensions the shape of the cytoplasmic channels along the axons. In the representative 3D reconstruction of myelination (chap 3.1.1.2) we could follow a system of cytoplasmic channels running through the compact myelin sheath for $30 \mu \mathrm{m}$ (red compartment in fig 3.26). We found that the cytoplasmic channels are running towards the two ends of the myelin segments from the oligodendrocyte process (arrow in fig 3.26). Furthermore, on one heminode we observed that the channels were ending in the inner tongue at two areas (fig 3.26). At these locations we found that the inner tongue winding appeared faster than along the rest of the myelin segment (fig 3.26). The appearance, the morphology and the spatial organization of the channels indicate that they could serve as shortcuts throughout the compact myelin sheath for the material to be delivered to the inner tongue during the most intense phase of the growth.

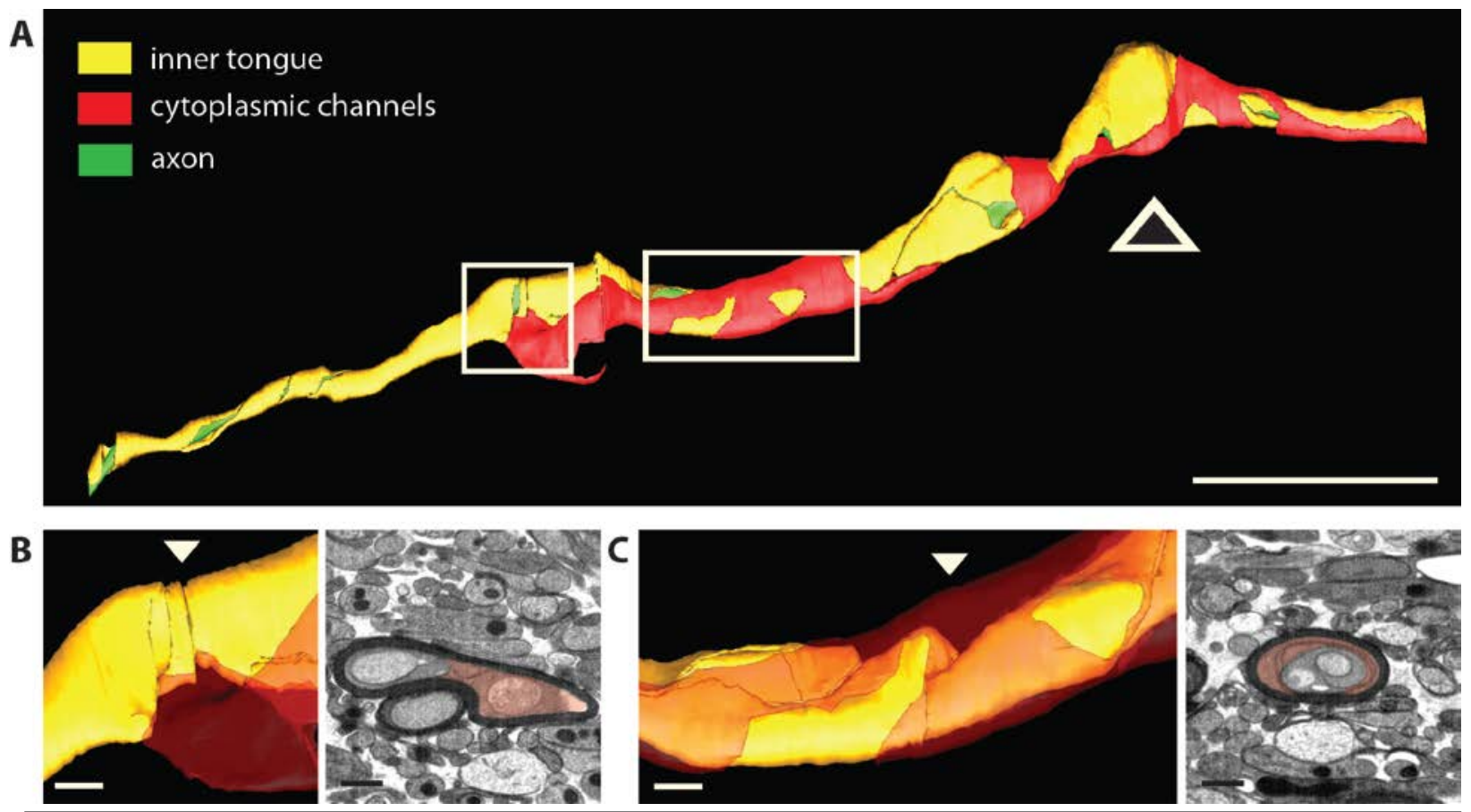

Figure 3.26: Three dimensional morphology of the cytoplasmic channels.

A) Three dimensional reconstruction obtained based on the FIBSEM (Fig 3.4) in which the cytoplasmic channels have been segmented in red. The channels are spreading along a large part of the myelin sheath towards the two ends of the segment (arrow is pointing the cell body location). Scale bar $10 \mu \mathrm{m}$. B,C) Enlarged views of the reconstruction with the associated electron micrographs of the areas where the cytoplasmic channels are reaching the inner tongue. Scale bar $1 \mu \mathrm{m}$. 


\subsubsection{Vesicular distribution along the non-compacted areas of growing sheaths}

We found that the cytoplasmic channels would provide a shortcut for the material delivery towards the inner tongue during the myelination process. Based on these findings, we asked whether the cytoplasmic channels could control a vesicular carrying for their putative function as transport highways. Using TEM cross section of optic nerve at P10, P14, P21 and P60 we determined the area covered by vesicular patterns at the inner tongue, outer tongue and cytoplasmic channels. We could see that the distribution of the vesicles was similar between all three compartments (both at P10 and P14 13-17 percent of the area were covered by vesicles) (fig 3.27). Between P14 and P21 we could observe a significant reduction of the area covered by vesicles at the inner tongue and outer tongue (fig 3.27). At P60, when the myelination process would be completed, the area covered by vesicles was even smaller in all three compartments (fig 3.27). The amount of cytoplasmic channels at P21 and P60 were so little that no quantification was possible for these time points. We could confirm these results by assessing the distribution of the vesicles at P10 within a full heminode using the FIBSEM (fig 3.27). We found a relatively homogeneous distribution of the vesicles along the axon and a clear positive correlation between the area of the compartments and the amount of vesicles present. 

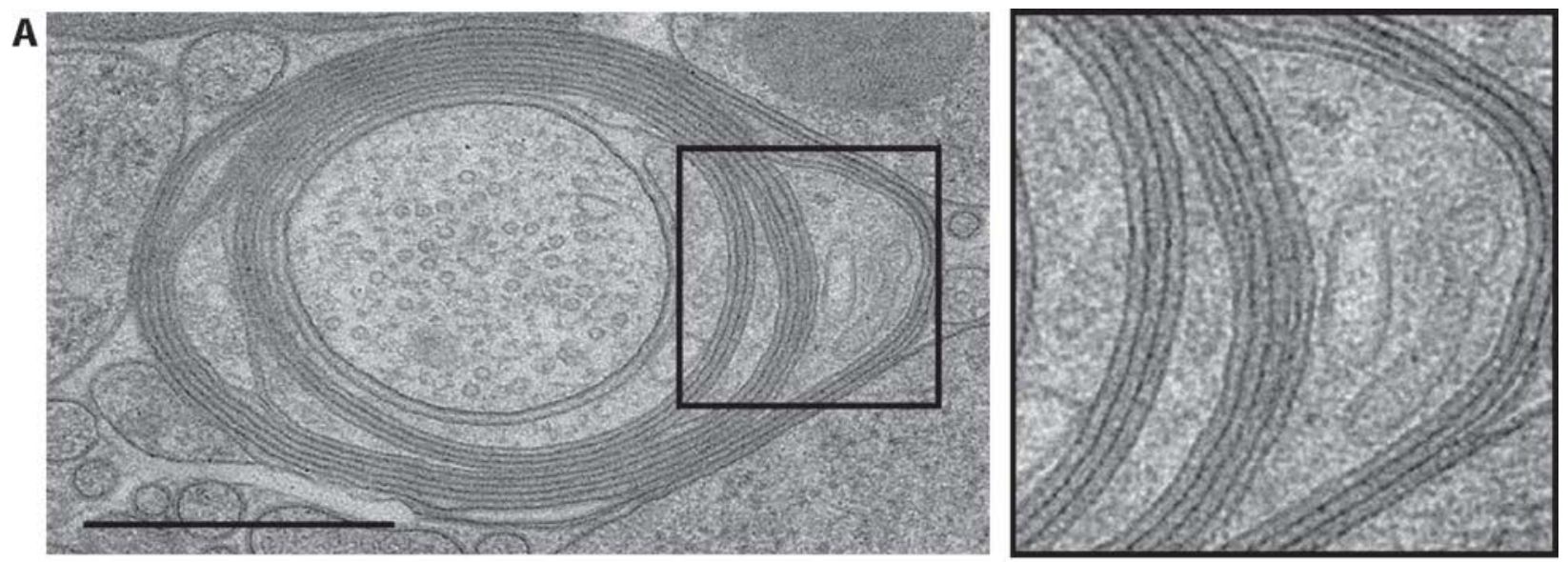

B
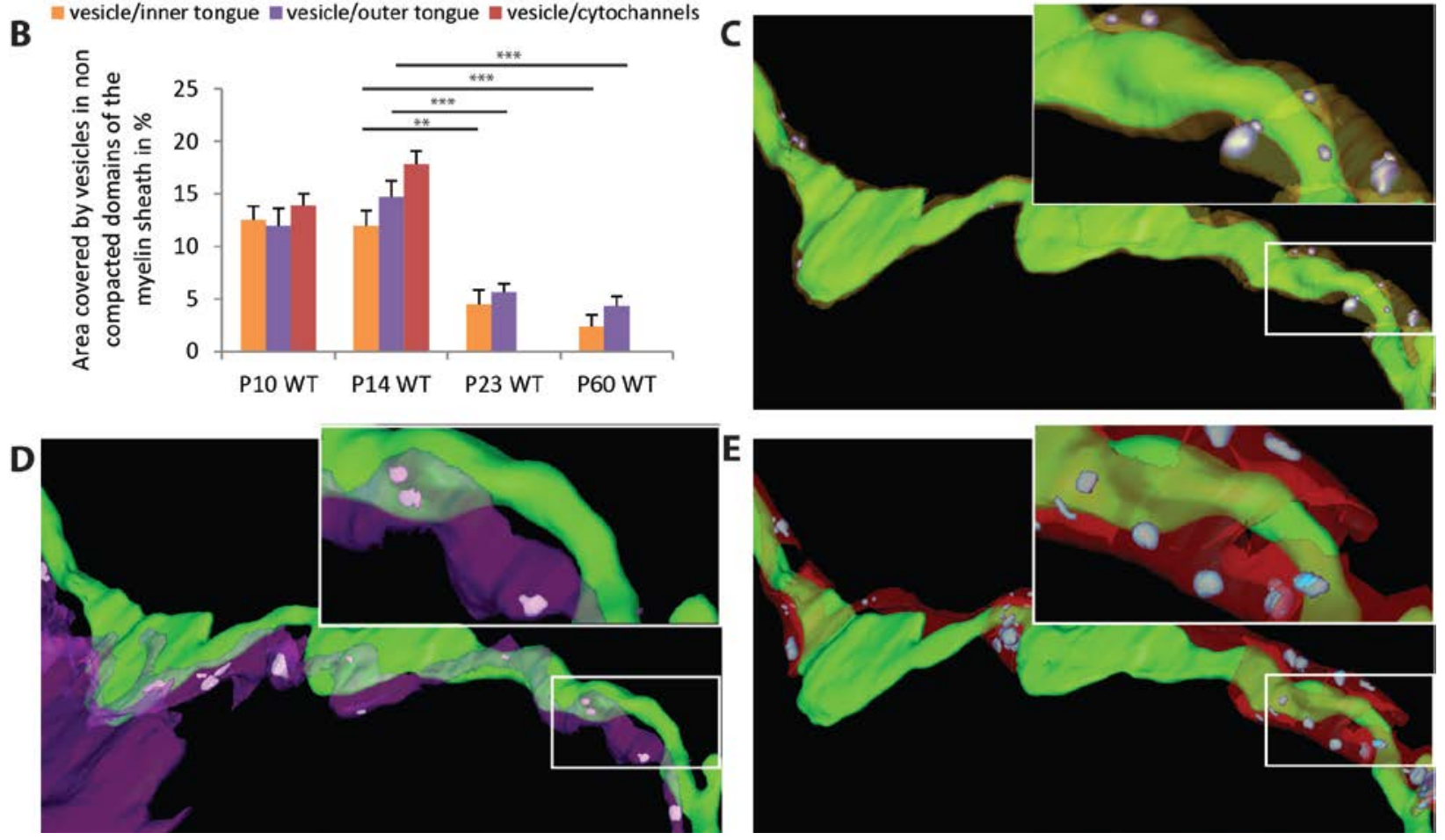

Figure 3.27: Vesicular profiles distribution within the myelin sheath during its maturation.

A) Electron micrograph of P10 HPF optic nerve showing multiple vesicular profiles within the non-compacted areas of the growing myelin sheath. B) Quantification of the area covered by vesicular patterns within inner tongue, cytoplasmic channels and outer tongue during the myelin maturation showing that the areas covered by the vesicles greatly decrease with the myelin development. C-E) Three dimensional reconstructions of a growing myelin sheath illustrating the distribution of vesicular profiles segmented within non-compacted myelin domains at P10 (inner tongue (orange), cytoplasmic channels (red), outer tongue (purple)). Bars show mean \pm SD ( $\mathrm{n}=3$ animal, 50 axons per animal). Scale bar $500 \mathrm{~nm}$. 


\subsubsection{Redundant myelin during the myelin biogenesis}

During our investigations of the cytoplasmic channels and vesicle distribution we found another frequent morphological feature, where the myelin sheath would form outfoldings of compacted myelin layers. These outfoldings, or redundant myelin, have been previously described in myelin pathologies including dysmyelinating neuropathies (Pereira et al., 2013). In this study, based first on the spatial investigation of the sheath morphology by FIBSEM and next on TEM cross sections, we found that the large amounts of myelin sheaths were presenting redundant myelin during development. The 3D reconstruction of the sheaths with redundant myelin showed that the sheaths themselves are normally compacted and the myelin thickness is in continuity with the areas before and after this outfolding. We could also see that at the tip of the outfolding, a pocket of cytoplasm, sometimes mistaken for an axon, is almost always present (fig 3.28). We could observe in $6.7 \pm 2.2$ percent of the myelinated axons, assessed in TEM cross sections, outfoldings at P10 (fig 3.28). During maturation the amount of outfoldings per myelinated axons would rapidly being reduce to $2.8 \pm 0,7$ percent at $P 14,0.7 \pm 0.2$ percent at P21 and to $0.2 \pm 0.01$ at P60 (fig 3.28), suggesting that the outfoldings formation and disappearance is a normal feature of myelination.

Conjointly with the reduction of the amount of outfoldings we found, using cryo-immuno electron microscopy, that the amount of Claudin11, a part of the radial components that stabilize the myelin sheath, would increase with time. At P10 barely any labeling of Claudin 11 could be found when at P14 some proteins could be detected all around the compacted sheath. It is only starting from P21 that Claudin11 would accumulate at defined areas within the compact myelin and at P60 we could visualize a large amount of these proteins clustered in a small portion of the sheath (fig 3.28).

Figure 3.28: Myelin outfoldings in the developing myelin sheath.

A) Serial electron micrographs of a myelin outfolding spreading over $10 \mu \mathrm{m}$ obtained by block face imaging. The axon is highlited in green, the inner tongue in orange, the compact myelin in cyan, the outer tongue in purple and the cytoplasmic channels in red. B) High resolution TEM micrograph on which the myelin layers can be followed along the outfolding (black arrow) showing that the layers are continuous along this structure. At the tip of the outfolding a cytoplasmic channel can be seen (star). C) Quantification of the amount of myelinated axons presenting myelin outfoldings during development between P10 and P60. D-F) Immuno electron micrographs of P10 (D), P21 (E) and P60 (F) optic nerve for OSP illustrating the accumulation of the Claudin 11 protein at the radial component after the main pick of myelination (arrows). Bars show mean \pm SD ( $n=3$ animal, 200 axons per animal). Scale bar $500 \mathrm{~nm}$. 

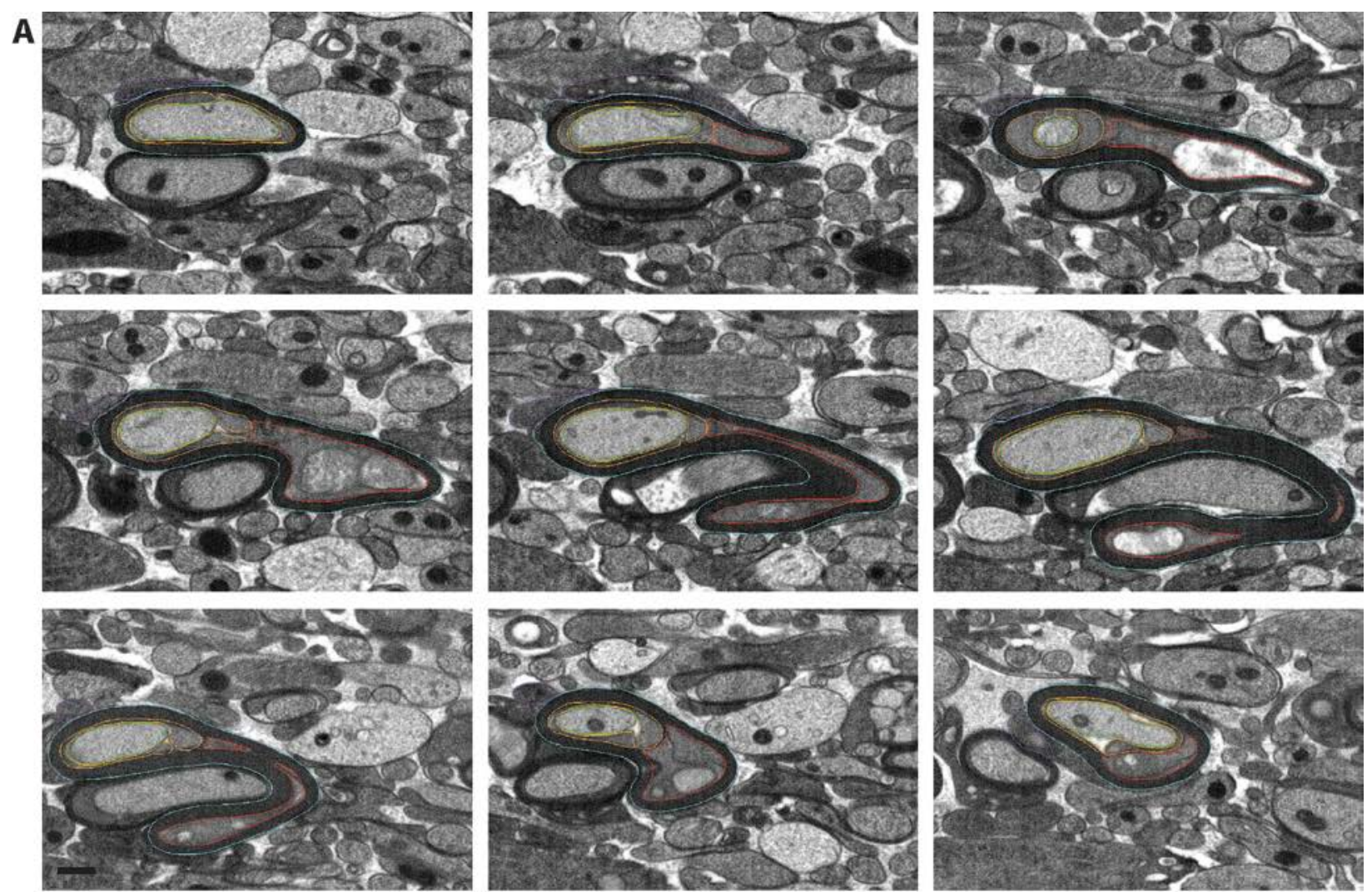

B

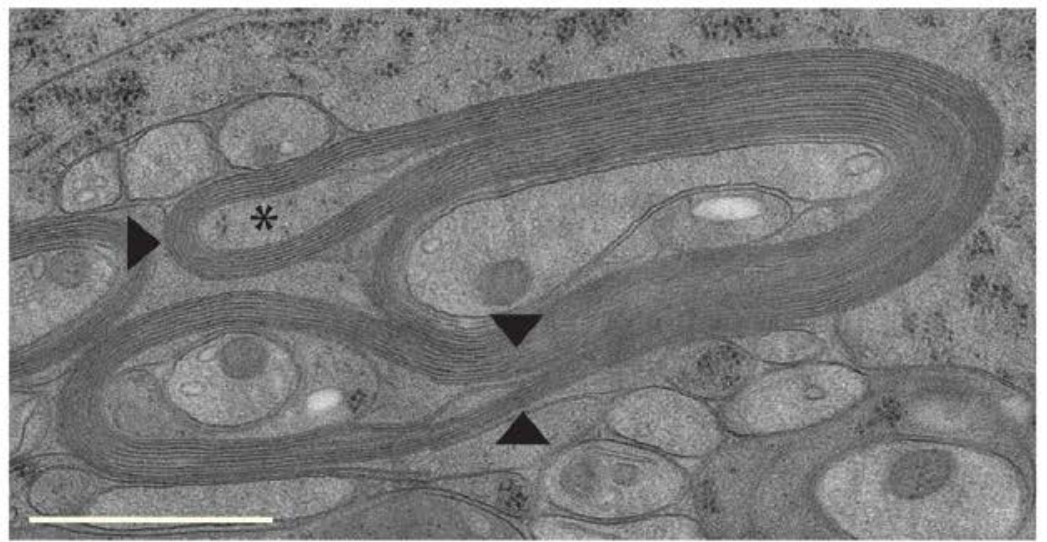

C outfoldings $\longrightarrow$ myelinated axons

D
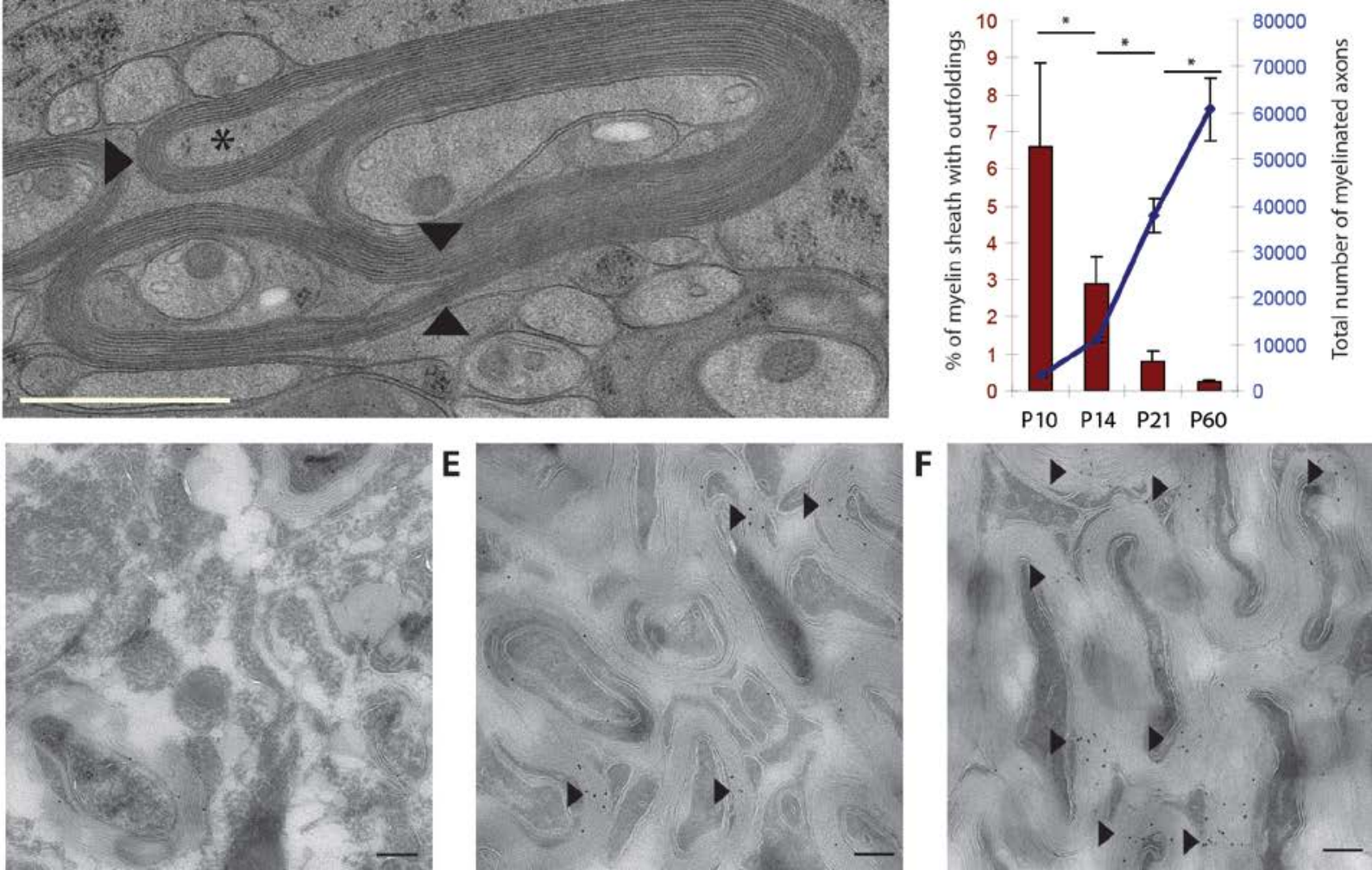
Based on the FIBSEM data we further characterized the spatial features of these outfoldings and found that the average length of the outfoldings within the assessed volumes was around $10 \mu \mathrm{m}$ (fig 3.29). Thus, if an internode is in average $100 \mu \mathrm{m}$ long and 6.5 percent of the myelin sheath at P10 show outfoldings in cross sections, we concluded that almost 65 percent of the internodes would present outfoldings.

Interestingly, we found that most of the outfoldings within the volumes imaged by FIBSEM where located at the side where cytoplasmic channels ended (fig 3.29). Indeed, the cytoplasmic channels would run through the cytoplasmic rich area at the tip of the structure and would reach the inner tongue at the lateral end of the outfolding (fig 3.29).
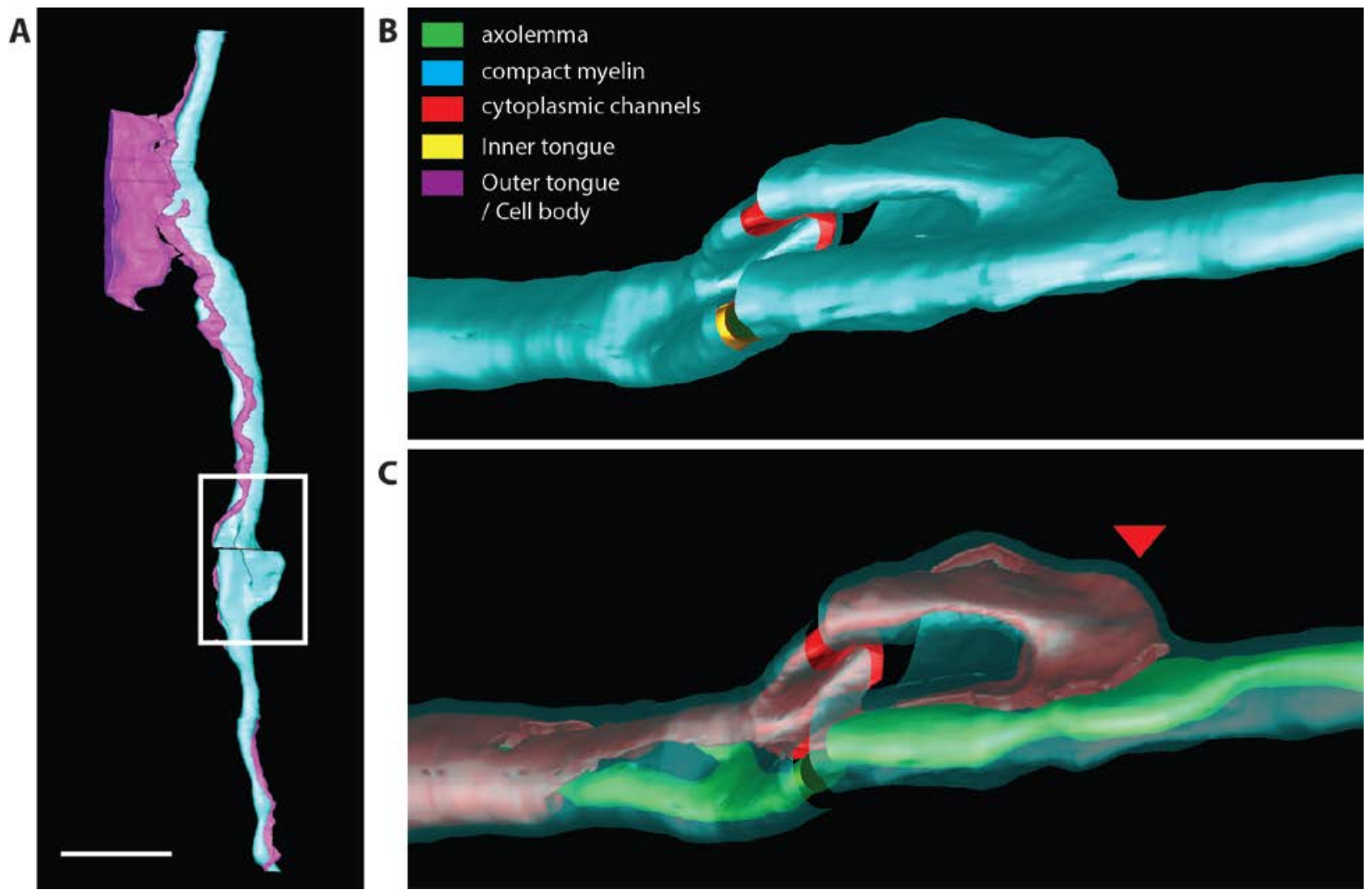

\section{Figure 3.29: Three dimensional view on the outfoldings}

A) Reconstruction of the P10 myelin sheath (Fig 04) indicating the area where the outfolding spans (white frame). B-C ) Enlarged views of the outfolding in three dimensions with a surface view (B) and a transparent view (C) allowing the visualization of the cytoplasmic channels running through the outfolding and ending up at the lateral edge of the myelin outgrowth (red arrow). Scale bar $10 \mu \mathrm{m}$. 
Based on the morphology of the layers and the position of the cytoplasmic channels within the outfoldings we made a hypothetical model of outfolding formation during the myelin development. Material would be brought by the cytoplasmic channels focally to the inner tongue where it would be added to the growing myelin sheath. The lateral extension of the layer at this particular point would be slower than the speed at which the material is added, resulting in an accumulation of membrane that could extend only vertically and, thus create an outfolding.

Taken together, our observations support the idea that the cytoplasmic channels are facilitating the material delivery to the inner tongue which can lead to the transient formation of focal hyper myelination along the myelin segment during development.

\subsubsection{MBP mediated compaction during the myelin growth}

So far we could show that the inner tongue is the area where the newly synthesized material is transported and incorporated via the delivery through the cytoplasmic channels. However, another important aspect of myelin maturation is the compaction of the cytoplasmic leaflets of the opposing membranes from the growing layers, required to achieve a tightly packed stack of membranes of the mature sheath. Indeed, an organized compaction is required to achieve a properly compacted sheath without interfering with its growth. In this part of the study we wanted to assess whether the growth and compaction phenomenon were linked and to investigate the compaction processes of the growing myelin sheath.

\subsubsection{Gradient of compaction within the growing myelin sheath}

To answer these questions we focused our investigation of the compaction state of the growing myelin sheath over time by TEM analysis. Interestingly, we found that, in addition to the inner and outer tongues that represent the major non-compacted areas in a myelin sheath, a large amount of myelin sheaths had layers that were not compacted (fig 3.30). Indeed, we found that $12.1 \pm 3.0$ percent of the myelin sheath at P10 have non-compacted layers. However, at P14 only $1.6 \pm 0.2$ percent of myelin sheath would present non-compacted wraps and at P21 there was an even further decrease $(0.9 \pm 0.9)$ (fig 3.30). The cytoplasmic spacing of these non- 
compacted wraps was strikingly reproducible, we found that in average the distance between the two opposite membranes at these areas was $12.8 \pm 1.1$ nanometers. Interestingly, in addition of the constant spacing we found these non-compacted wraps of myelin to be invariably located at the inner most layers (fig 3.30). We also found that at P10, the thickness of the myelin positively correlates with the number of uncompacted inner layers (fig 3.30), with only one non-compacted wrap from the inner tongue in the sheaths with 5 wraps up to 5 noncompacted layers from the inner tongue in the sheaths with 11 wraps. In the 4 wraps thick myelin we could see in more than 50 percent of the analyzed sheath that the entire sheath was non-compacted, and in the other 50 percent the non-compacted layers would always be present next to the inner tongue.

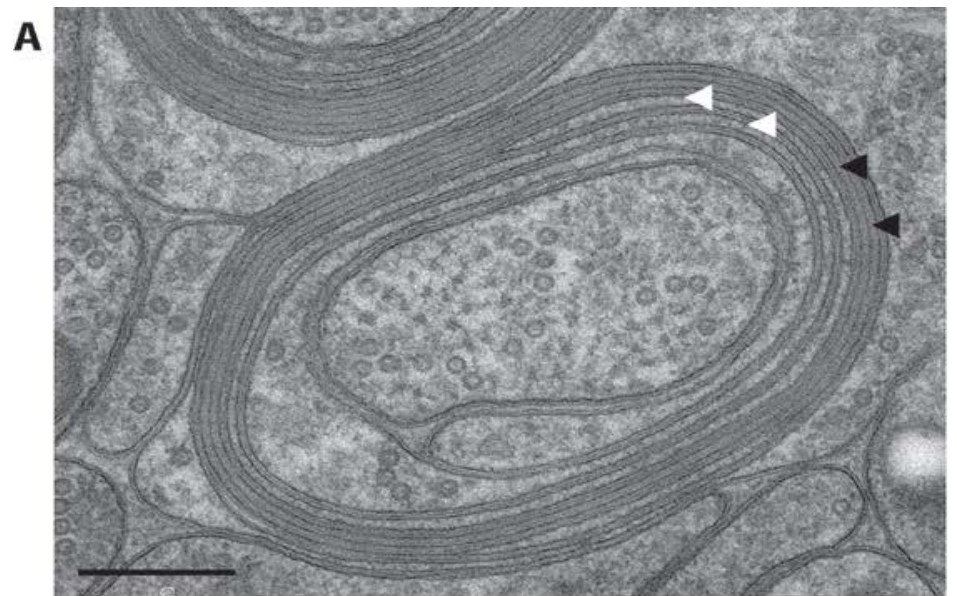

B

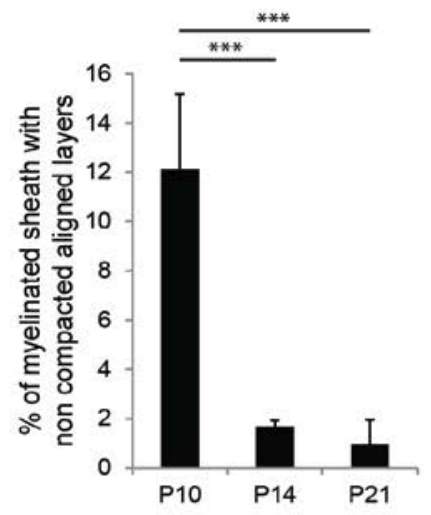

C

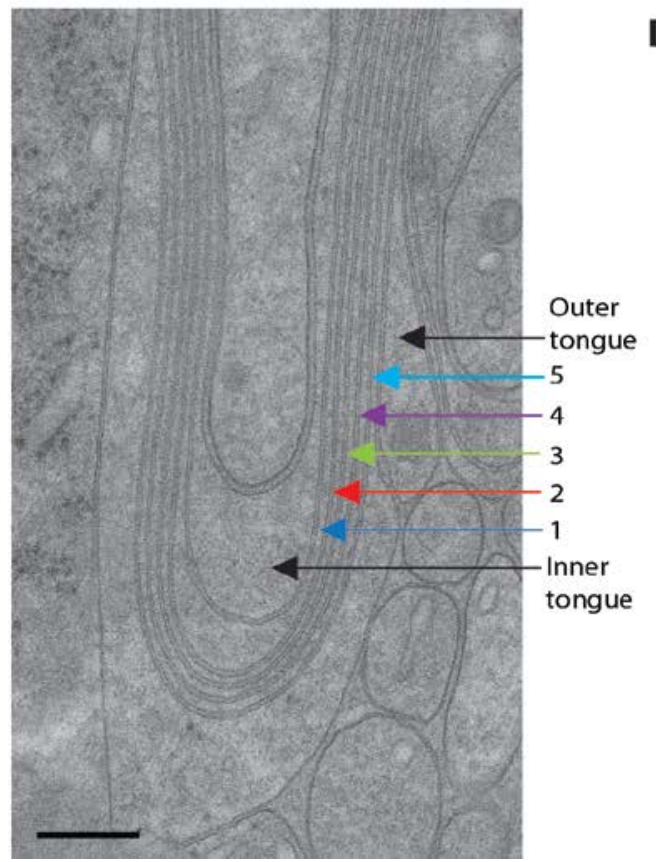

D

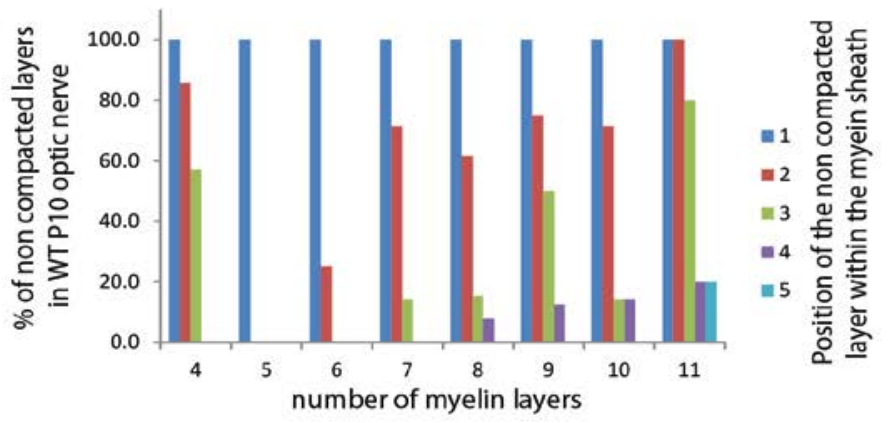


Figure 3.30: Compaction of the growing myelin sheath.

A) P10 optic nerve myelinated axon showing a gradient of compaction within the sheath, with the outer layers compacted (black arrows) and the two layers after the inner tongue non-compacted (white arrows). The spacing of these two uncompacted wraps appears constant until it reaches the inner tongue. B) Quantification of the amount of myelinated axons presenting non-compacted aligned wraps within the myelin sheath (excluding inner and outer tongue) during myelination (3-5 animals, 200 axons per animal). C) Electron micrograph of a fully uncompacted myelin sheath used to localize within the sheath (various colors) and name (with number) the non-compacted wraps. This classification was used in D) for localizing and quantifying the frequency of the non-compacted aligned wraps in function of the thickness of the myelin sheath at P10. Bars show mean \pm SD ( $n=3$ animal, 200 axons per animal). Scale bar $200 \mathrm{~nm}$.

\subsubsection{Growth and compaction are independent processes in the early stages of myelination}

Since MBP is the essential protein for the cytoplasmic compaction, we wanted to assess its distribution within the two different compaction states of the myelin sheath. Using immuno EM we observed that the non-compacted myelin layers would present four folds less MBP molecules than the compacted layers (fig 3.31). Since MBP is known to be critical for the compaction we used the heterozygote shiverer mouse, which have only one MBP allele and express half of the normal amount of MBP. The electron microscopy analysis of the shiverer heterozygote optic nerve at P10, P14 and P21 showed a drastic increase in the myelin sheaths with non-compacted layers at P10 and P14 (almost two folds) (fig 3.31). Interestingly, at P21 no significant difference could be observed as compared to wild type, confirming that the mature myelin sheaths in shiverer heterozygote mice have normal morphology. We found that the distribution of the non-compacted wraps in shiverer heterozygote myelin was similar to the wild type. However, around 80 percent of the sheaths with 4 wraps were completely uncompacted as compared to 50 percent for the wild type. We could also observe totally uncompacted sheaths up to 8 wraps of myelin thickness (fig 3.31), when the amount of myelinated axons and myelin thickness would remain unchanged between the two genotypes, indicating certain independence between compaction and growth. To further test this hypothesis we compared the amount of myelin at the early stages in shiverer homozygote in comparison to the wild type. We could see that even without MBP the initiation of the myelination happened relatively normally and, when assessing at P10, we found that the 
average thickness of the myelin sheath in shiverer homozygote was around 3 wraps, whereas 5 wraps were observed in wild type and heterozygote (fig 3.31). At later time point we found that the myelin sheath would become unstable in shiverer mice (fig 3.31). At P14 and P21 only 1.5 wraps of myelin could be found in average in the sheath for 7 and 8 respectively in wild type (fig 3.31). We could confirm previously published data showing fragmentation profiles in shiverer homozygote starting from P14 and more obviously at P21 (Shen et al., 1985), (fig 3.31). This data confirms that the initial wrapping steps are independent of the compaction of the myelin sheath. However, to proceed with the myelin growth, the presence of MBP is vital.

\subsubsection{Regulation of the compaction in the growing myelin sheath}

It has been suggested that MBP mRNA is transported to the inner tongue and that the translation process occurs at this compartment as well (Ainger et al., 1993); (Colman et al., 1982); (Trapp et al., 1987); (Wake et al., 2011); (Laursen et al., 2011). We also could localize in cell culture a component of the MBP mRNA granules, the hnRNP, in the cytoplasmic rich areas and we found that the protein was enriched towards the outer rim of the cultured oligodendrocytes (fig 3.32). In the light of these findings and previously published data, it was then surprising for us to observe that the inner most layers of the myelin sheath were noncompacted. Furthermore, considering the localization and amount of the MBP proteins within these compacted and non-compacted wraps we hypothesized that an active mechanism has to prevent the compaction at the inner most layers of the myelin sheath during the growth.

Previous studies based on CNP overexpression mutant showed similar myelin morphology with aligned, non-compacted wraps (Gravel et al., 1996). In this mutant the non-compacted layers would appear randomly within the myelin sheath and would show five folds less amount of MBP (Yin et al., 1997). Interestingly when investigating wild type P10 optic nerve we could find that CNP was present in these non-compacted layers as it has been described for the inner tongue and outer tongue in mature myelin sheath (fig 3.32), (Yin et al., 1997). We wanted to further investigate the role of CNP in the formation or maintenance of the non-compacted layers and for this purpose we looked at the compaction state of the myelin sheath in CNPdeficient mice. 


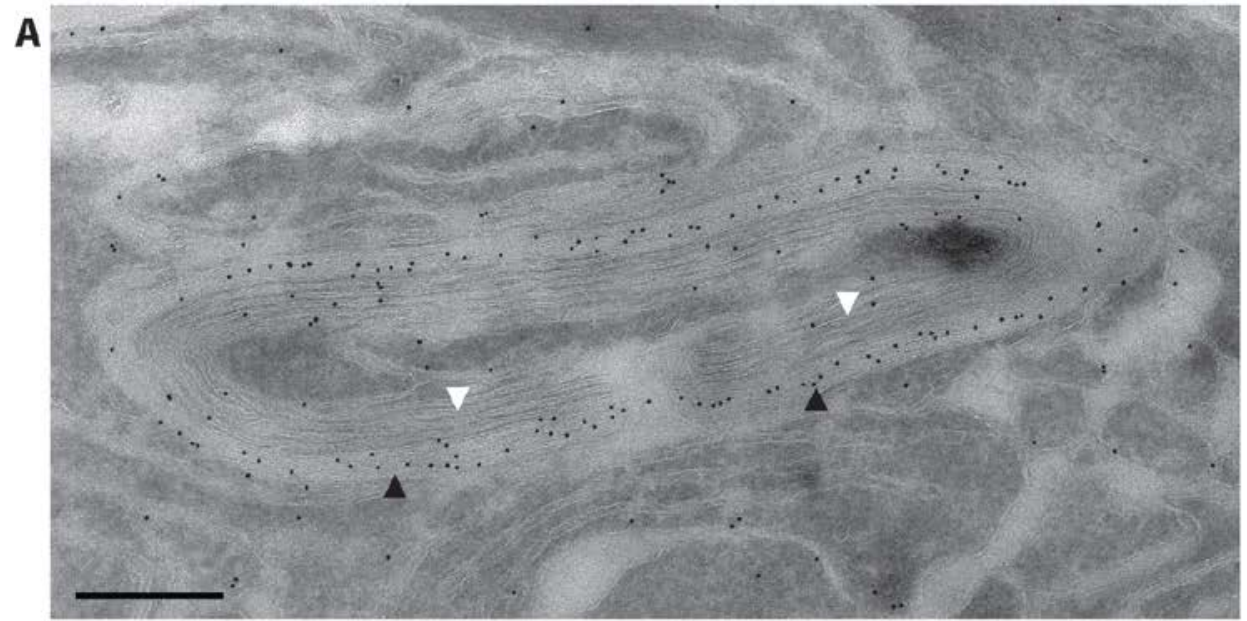

B

C

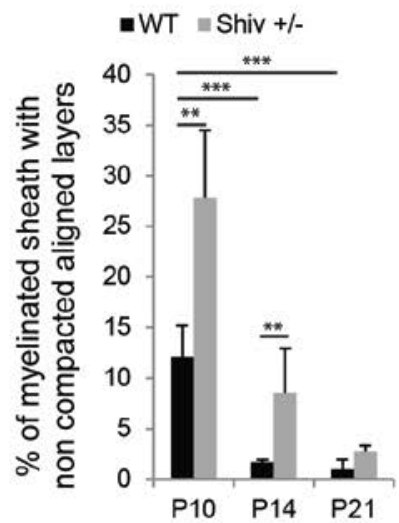

E

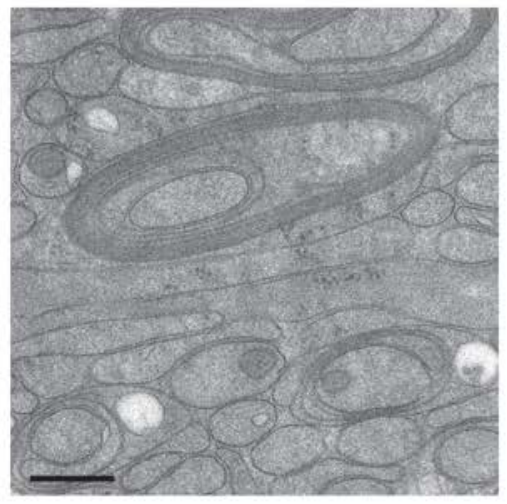

H

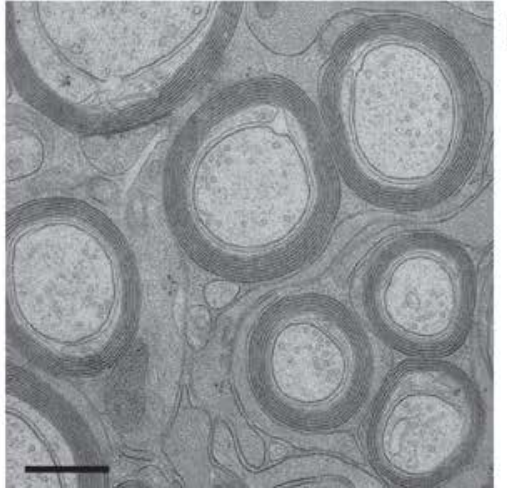

D
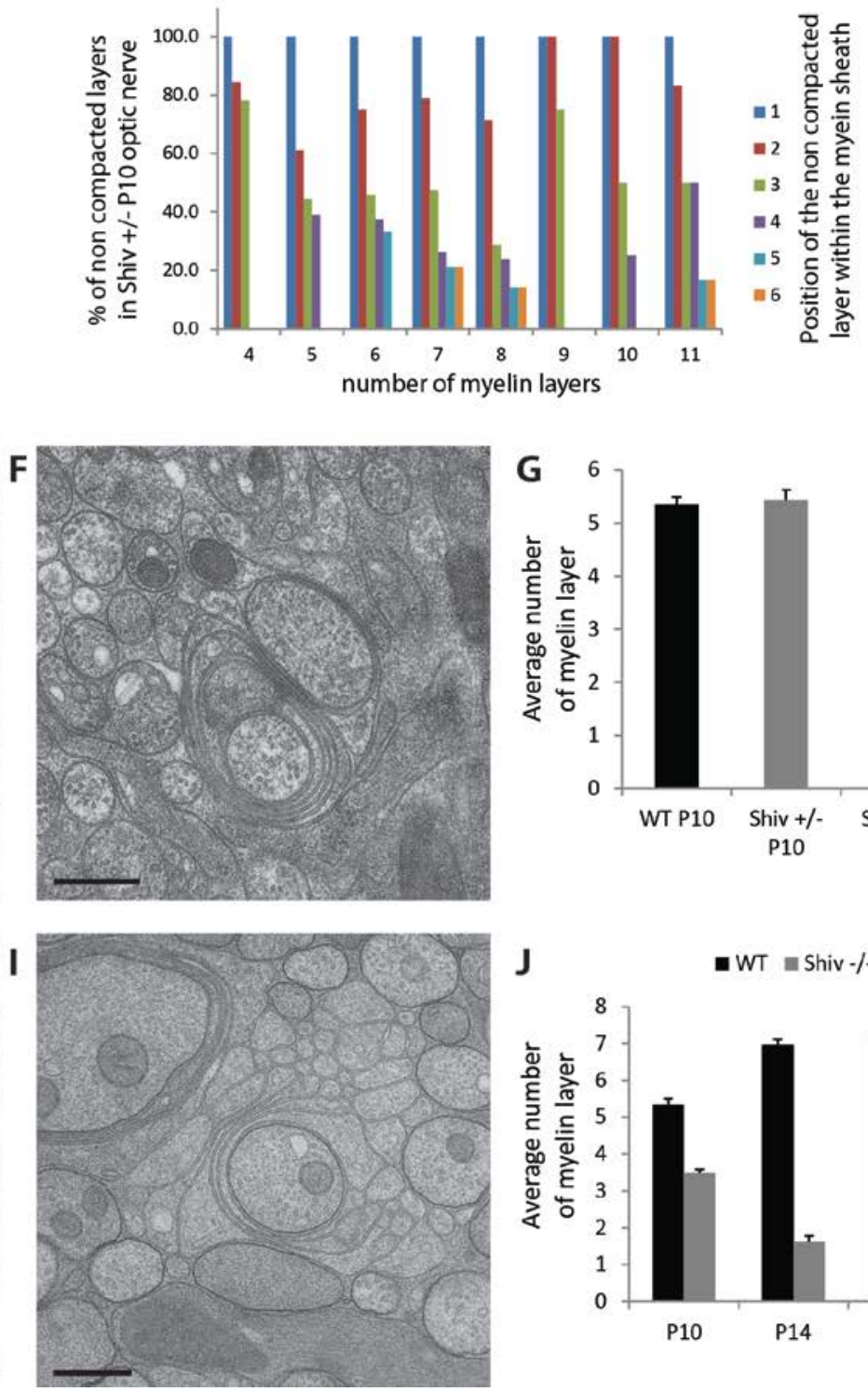
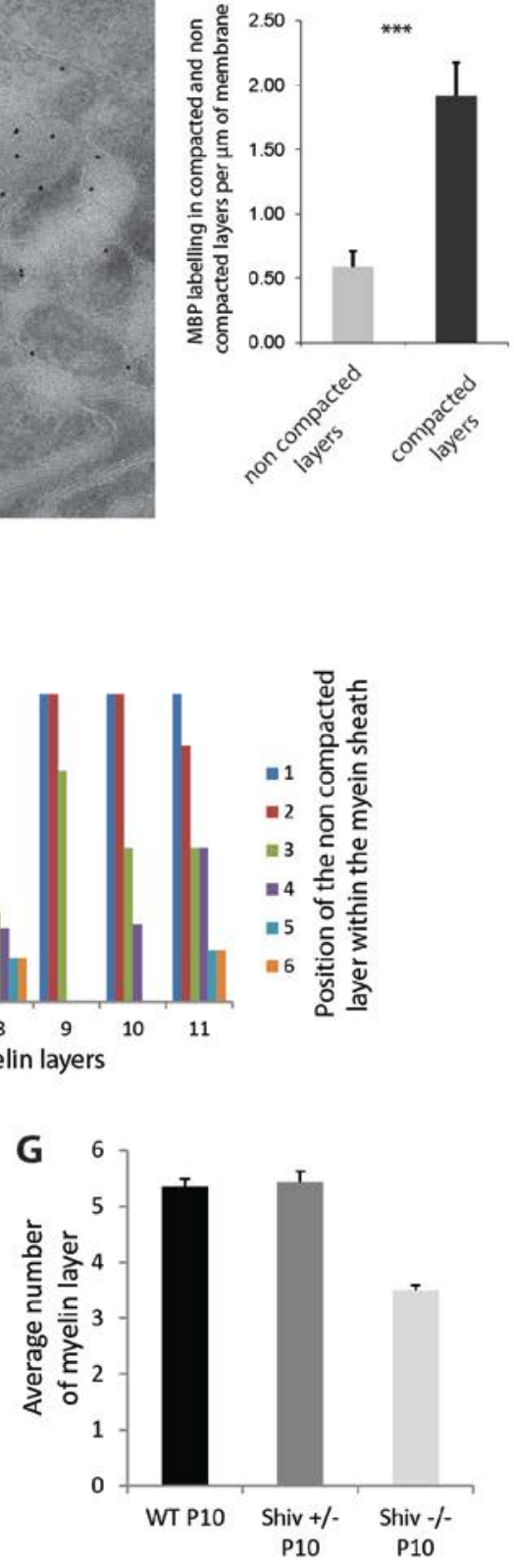

2 ᄃे है

4 这

5 은

6 壱

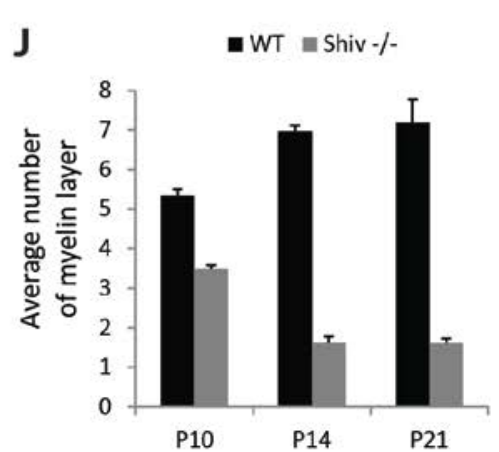


Figure 3.31: Effects of MBP levels on compaction and myelin stability.

A) Immuno electron micrograph of P10 optic nerve for MBP showing a large reduction of MBP protein labeling in the non-compacted aligned wraps (white arrows) compared to compacted layers (black arrows). B) Quantification of the MBP labeling per $\mu \mathrm{m}$ of membrane in compacted and non-compacted layers of P10 myelin sheaths. C) Quantification of the amount of myelinated axons presenting non-compacted aligned wraps within the myelin sheath (excluding inner and outer tongue) during myelination in wild type (data from Fig 30) compared to shiverer heterozygote (50 percent less MBP protein). D) Localization and quantification the frequency of the non- compacted aligned wraps in function of the thickness of the myelin sheath in P10 shiverer heterozygote mice. E, F) Electron micrographs of P10 optic nerve of wild type (E) and shiverer homozygote (no MBP protein) (F) showing only fully non-compacted myelin sheets. G) Quantification of the myelin thickness at P10 in shiverer heterozygote, homozygote and control. $\mathrm{H}, \mathrm{I})$ Electron micrographs of P21 optic nerve of wild type $(\mathrm{H})$ and shiverer homozygote (I) showing unstable myelin sheets with multiple oligodendrocytic micro processes. J) Quantification of the myelin thickness during myelination in shiverer homozygote compared to control. Bar show mean \pm SD ( $n=3$ animal, 200 axons per animal). Scale bar $500 \mathrm{~nm}$.

We found that with only 50 percent less of CNP expression we could already see a significant decrease in the myelin sheath presenting non-compacted layers with only 3,5 \pm 0.9 percent as compared to $25 \%$ in the wild-type. Furthermore, when assessing the CNP knockout we found that only $0.4 \pm 0.06$ percent of the assessed myelin sheaths had non-compacted layers (fig 3.32).

Taken all together, these results showed, based on the wild-type comparison to the shiverer heterozygote, that the growth and the compaction are, to a certain extent, independent processes. Furthermore, we found that compaction of the myelin sheath appears to start from the outer most layers of the myelin sheath. This phenomenon could be explained by the fact that myelin is growing from the inner tongue and that the CNP is preventing the premature compaction of the leading edge of the sheath by MBP.

\section{Figure 3.32: CNP is a possible regulator of the myelin compaction.}

A) Immuno staining of cultured oligodendrocyte for hnRNP showing an enrichment of the staining at the cellular rim compartment. Scale bar $20 \mu \mathrm{m}$. B) Immuno electron micrographs for CNP of P10 optic nerve myelinated axon with non-compacted aligned wraps (white arrow) positive for CNP labeling and compacted layers (black arrows) negative for the labeling. C) Electron micrograph illustrating the CNP distribution in a fully compacted sheath at the inner and outer tongue of the myelin only. D) Quantification of the amount of myelinated axons presenting non-compacted aligned wraps within the myelin sheath (excluding inner and outer tongue) at P10 in Shiverer heterozygote, CNP heterozygote, CNP homozygote and control mice. Bar show mean \pm SD ( $n=3$ animal, 200 axons per animal). Scale bar $200 \mathrm{~nm}$. 


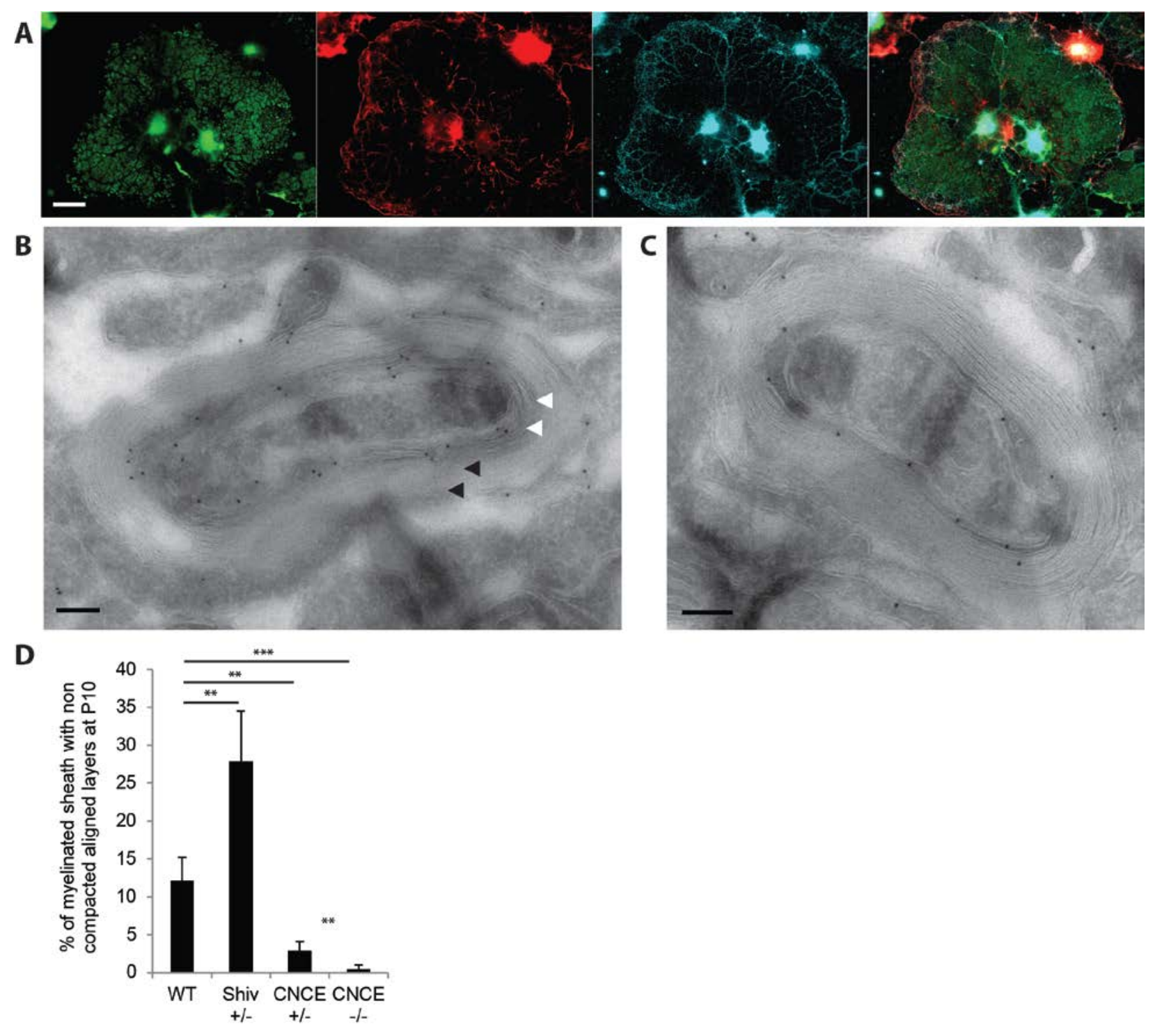




\subsection{Myelin sheath is an adaptive and plastic structure}

The myelin sheath is a very complex arrangement of spirally wrapped plasma membrane that is tightly compacted from their cytoplasmic face - by MBP and from their extracellular face by PLP and additional week interactions. Indeed, in previous studies we characterized how the intracellular and extracellular leaflets of myelin bilayers are brought together (Bakhti et al., 2013); (Aggarwal et al., 2011). Until recently the myelin sheath was thought to be a very rigid and stable structure. However, we could show that during development myelin shows features such as outfoldings, cytoplasmic channels or non-compacted layers that will disappear with the sheath maturation. Lately the concept of an adaptive myelin sheath throughout life grew and evidences of myelin plasticity in various environments have been reinforced (Liu et al., 2012); (Makinodan et al., 2012); (Yang et al., 2013).

In this study we investigated the morphology of a mature myelin sheath in adult where the growth was experimentally re-initiated and we assessed the physiological relevance of this plasticity. Again, we made use of a conditional PTEN knockout mutant where the enzyme was constitutively inactivated in oligodendrocytes (Goebbels et al., 2010). Now we used an inducible conditional PTEN knockout mutant in which the PTEN could be inactivated in oligodendrocyte at any time during development by Tamoxifen injection, leading to an increase in myelin thickness and formation of redundant myelin (Goebbels et al., 2012). We asked whether the re-initiation of the myelin growth in a stable mature sheath in adult would involve similar morphological features as the ones observed during the development, such as the cytoplasmic channels.

To investigate the effects of PTEN inactivation on the mature myelin, P100 Pten ${ }^{f l o x}$ flox* Plp1CreERT2 mutant mice were injected with Tamoxifen for 10 days and further processed for EM 30 days or 90 days after the last Tamoxifen injection.

We found, in the mature myelin of the optic nerve in PTEN KO mice, that $11.3 \pm 0,6$ percent of sheaths would present cytoplasmic channels after 30 days in TEM cross sections compared to only $1 \pm 0.1$ percent in control Pten ${ }^{f l o x / f l o x} *$ Plp1-CreERT2 mice injected with corn oil. Interestingly, we could observe that after 30 days of PTEN inactivation the average myelin sheath thickness was not significantly bigger in the optic nerve (fig 3.33). 
When assessing 90 days after Tamoxifen injection we still found $10,8 \pm 1,8$ of cytoplasmic channels within the sheaths, but now we could observe a very significant increase in myelin thickness (fig 3.33).

Thus, cytoplasmic channels are re-opening when growth is re-initiated in the adult myelin in response to the increase of the PIP3 levels, confirming the myelin plasticity in adulthood.

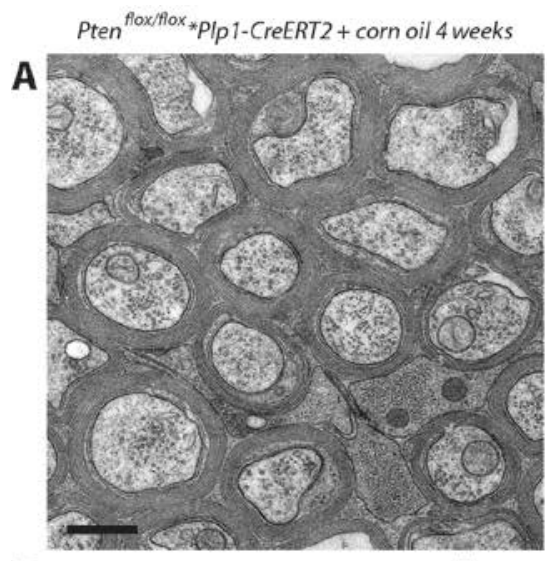

D

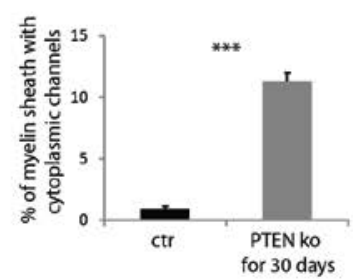

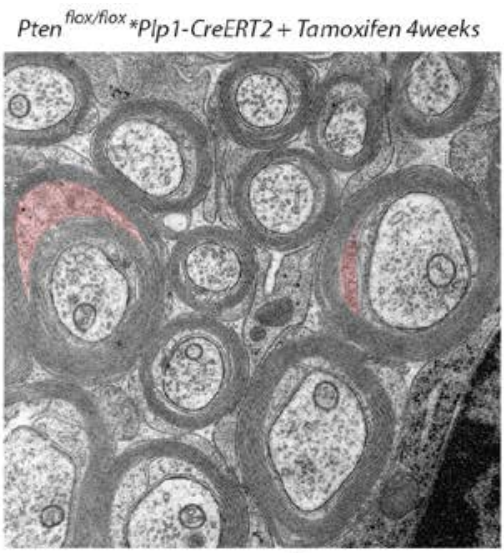

F
Pten flox/lax $*$ Plp 1-CreERT2 + Tamoxifen 12weeks

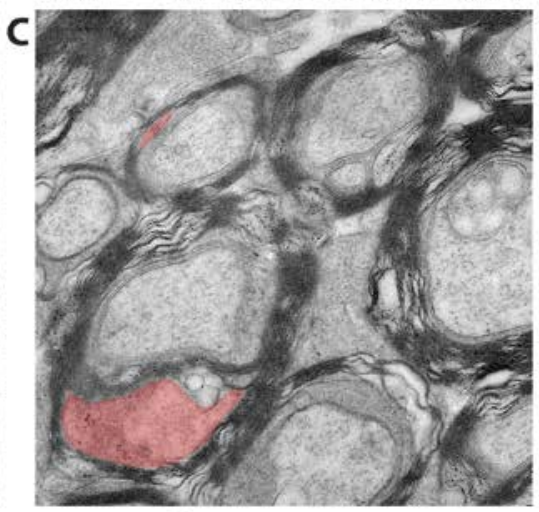

G
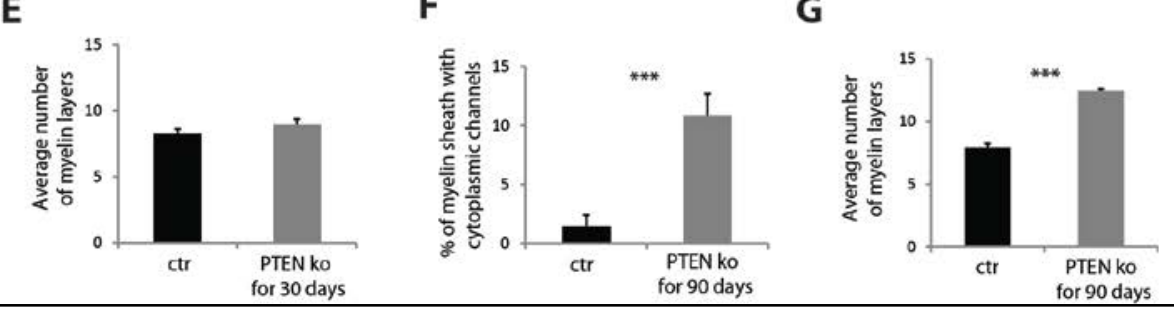

Figure 3.33: Interconnection between the cytoplasmic channels and the myelin growth.

A-C) Electron micrographs of the adult PTEN flox/flox *Plp1-CreERT2 mutants injected with corn oil (A) and Tamoxifen (B, C) and sacrificed after 4weeks (A,B) or 12 weeks (C). D) Quantification of the amount of myelinated axons presenting cytoplasmic channels 4 weeks after tamoxifen treatment and in control situation. E) Quantification of the average myelin thickness in optic nerve myelinated axons of animals 4 weeks after tamoxifen treatment and in control situation. F) Quantification of the amount of myelinated axons presenting cytoplasmic channels 12 weeks after tamoxifen treatment and in control situation. E) Quantification of the average myelin thickness in optic nerve myelinated axons of animals 12 weeks after tamoxifen treatment and in control situation. Bars show mean $\pm S D$ ( $n=3$ animal, 100 axons per animal). Scale bar $500 \mathrm{~nm}$. 


\section{Discussion}

The aim of this study was to understand the mechanisms underlying the axon myelination in the central nervous system.

The myelin sheath presents one of the most complex cellular morphology achieved by a single cell. In the CNS, one single oligodendrocyte can produce up to 50 myelin sheaths (Peters and Proskauer, 1969) of around $200 \mu \mathrm{m}$ length (Ransom et al., 1991) composed by a tightly compacted spiral of membrane that is wrapped several times (up to 50 times) around the axon. Although the myelin sheath has been studied at nanometer range scale for more than 60 years, several fundamental questions remain to be answered. It is still unclear how the myelin sheath forms or where the newly synthesized material is added to the growing myelin sheath. Furthermore, relatively little is known about how the growth and compaction of the myelin layers are related.

Based on diverse approaches such as multiple imaging techniques, molecular tools and mouse mutants, we could establish a novel model of myelin membrane growth in the central nervous system (fig 4.1, video 05).

In this model we propose that the myelin sheath is growing by two distinct and coordinated motions with the first being a radial growth that is achieved by the winding of the inner tongue under the previously deposited membrane in the center of the myelin segment. The myelin sheath shows a triangular shape leading to the structure where every lateral cytoplasmic domain of the layers are in contact with the axon in a helical manner towards the node of Ranvier, with the outermost layer covering almost the entire length of the sheath. Concurrently to this radial growth the successive layers extend laterally towards the node of Ranvier leading to a transition from a triangular to a rectangular shaped sheath during its maturation (fig 4.1). We also could identify that the insertion of the newly synthesized material from the oligodendrocyte was happening at the inner tongue and we could characterize cytoplasmic channels that would go through the compacted myelin during the most intense growth phase of the sheath, providing "shortcuts" for the transport of the newly synthesized components 
from the cell body of the glia cell to the inner tongue. Most of the cytoplasmic channels appear transiently during development and are regulated by the level of PI(3,4,5)P3 expression.

In addition, we could observe that, while the growth would occur at the inner tongue, the compaction of the intracellular domain of the myelin sheath by MBP would progress from the outer layers towards the inner tongue spatially separating these two processes.

\subsection{Tools for high resolution morphological analysis}

In order to investigate the myelin biogenesis, the most important was to understand the exact morphology of the myelin sheath at various time points during its development. To achieve this goal we prepared mouse CNS tissue using high pressure freezing for electron microscopy. The sample preparation for this technique has been described to give a close to native preservation and thus, was the most suitable to avoid fixation artifacts (Mobius et al., 2010).

The myelin sheath presents a great difference of scale when it comes to its morphology: it is a tightly compacted spiral of membrane with only around $12 \mathrm{~nm}$ spacing between neighboring layers, but, at the same time, the myelin segments would spread along the axon for up to 200 $\mu \mathrm{m}$. This very large difference in scale magnitude made the investigation of the myelin morphology challenging. To overcome these inherent complications we used serial block face imaging of high pressure frozen optic nerve by scanning electron microscopy to acquire a large volume of tissues at nanometer scale.

By combining volume microscopy with conventional TEM of high pressure frozen optic nerve at different time points of development we could reconstruct the 3D organization and follow the changes of the myelin sheath during its growth.

\subsection{Morphology of the growing myelin sheath in mammalian CNS}

The three dimensional reconstructions of myelination events, segmented from the P10 high pressure frozen mouse optic nerve, as well as our TEM studies, allowed us to follow the positioning of the inner and outer tongue along the growing myelin sheath. Indeed, we could visualize the oligodendrocyte cell body in direct contact with the outer tongue (fig 4.1). 
A
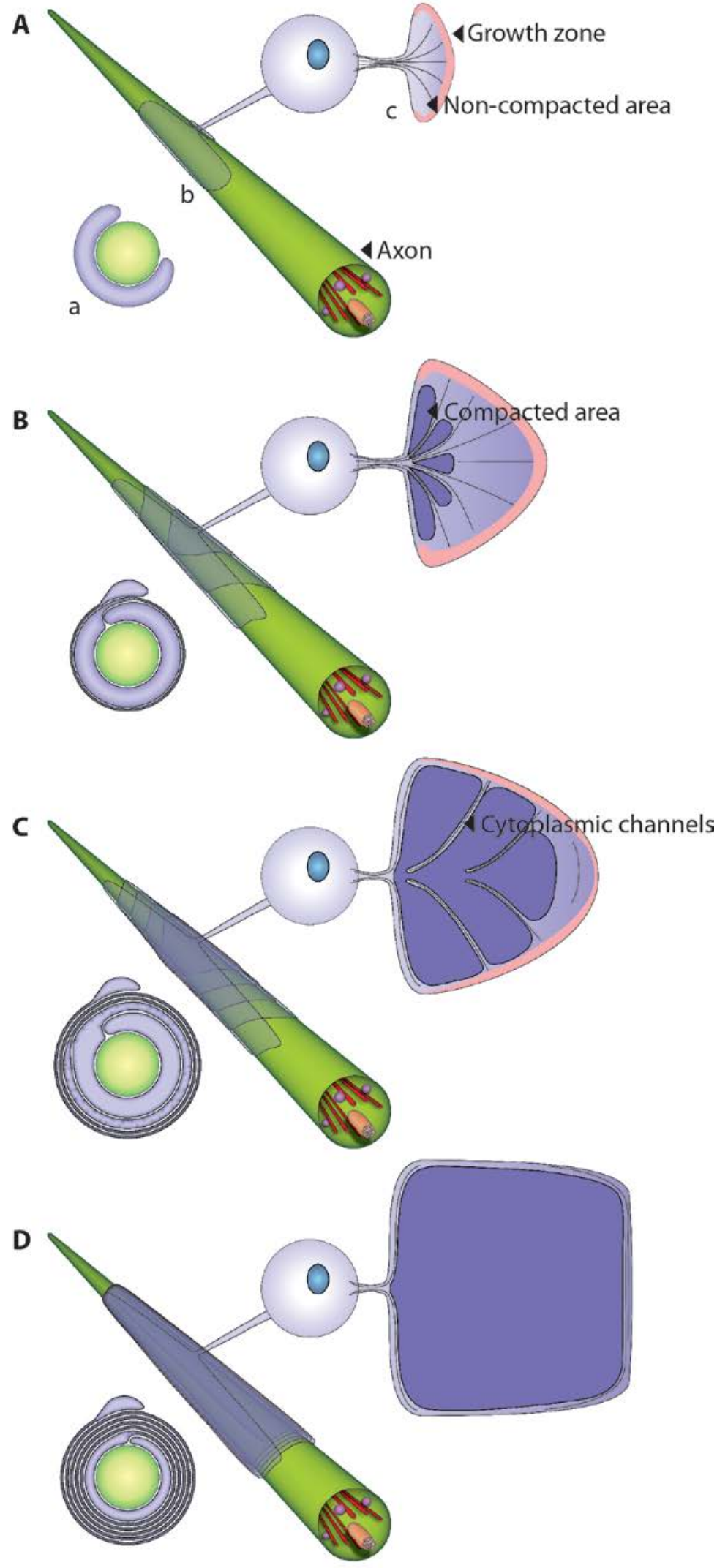

Figure 4.1: Model of CNS myelination.

Schematic representation of the myelin growth in the central nervous system decomposed in a cross sectional view (a), a three dimensional view (b) and an unwrapped view (c). A) Early stage of the myelination with the oligodendrocyte contacting and circling the axon by a single uncompacted wrap. The growth zone is colored in pink, the non-compacted cytoplasm in light violet and the axon in green. B) The myelin sheath grows from the leading edge at the inner tongue underneath the pre-deposited layers. The MBP accumulation starts the compaction process from several nucleation points (dark violet areas). C) The growth and compaction are proceeding, leaving the inner most layers of the sheath uncompacted, and the cytoplasmic channels, present within the compacted areas allow the delivery of the newly synthesized material at the leading edge. D) The growth of the sheath is over and the layers extended laterally to the nodes of Ranvier leading to a mature myelin sheath. 
The outer tongue would barely wind around the myelin sheath, covering most of the internodal length with one wrap of myelin. In addition, we observed myelin thinning from the area where the oligodendrocyte contacts the sheath towards the lateral edge of the segment. We found that the inner most layer of the myelin was centered in front of the oligodendrocyte process contact and that it would have the shortest length. Each successive layer towards the surface of the sheath would build up in size resulting in a myelin sheath with the thickest part in the center. With such morphology the unrolled sheath would look like a triangle with its base being the outer tongue and its tip being the inner most layer of the sheath. Based on the 3D reconstructions we could follow the cytoplasmic-rich area at the lateral end of the layers contacting the axon and found that it would form a coil along the segment of relatively

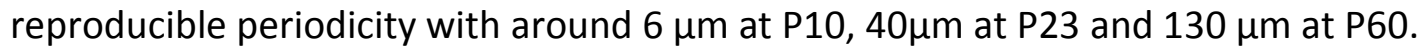

In the previously published myelination studies a spiral or ribbon of cytoplasm has often been described during the growth phase of the sheath (Berry et al., 1995); (Pedraza et al., 2009); (Sobottka et al., 2011); (loannidou et al., 2012). However, some of these studies claim that the helical pattern comes from oligodendrocytic processes that would coil around the axon similarly to a cork screw and then extend laterally covering each other (Pedraza et al., 2009); (Bauer and ffrench-Constant, 2009); (loannidou et al., 2012). According to another interpretation, the imaged coil represents the contours of the outer face of the sheath coiling along the myelin segment (Berry et al., 1995); (Sobottka et al., 2011). The frequency of coiling described in these investigations would correlate with our observations, but due to the fact that these studies were almost exclusively based on light microscopy (Pedraza and Colman, $2000)$ and considering the diffraction limit of light $(200 \mathrm{~nm})$ it is possible that the positioning of the coiling was not accurately assessable leading to inaccurate views of the myelin process. Furthermore, GFP/YFP tagged proteins were most often used in these studies and we could show that, due to their large cytoplasmic domains, these proteins would be extruded from the compacted sheath leading to a labeling sequestration to the cytoplasmic rich areas that would border the compact myelin sheath (inner tongue, outer tongue, cytoplasmic channels and paranodal loops) (Aggarwal et al., 2013; Aggarwal et al., 2011). 
To achieve a resolution sufficient to distinguish the positioning of the individual myelin layers the electron microscopy imaging is required. However, due to the sample size restriction for the TEM, EM volume microscopy techniques have to be applied to grasp the myelin morphology along the segments. Knobler and colleagues (Knobler et al., 1974, 1976) were the first to use TEM serial sections of CNS tissues to assess myelination. They could find vermicular processes within the sheaths and postulated that oligodendrocyte processes would wind around the axon like a "thread of screw". It is mainly based on these observations that the light microscopy studies mentioned above built their models. The studies of Knobler and colleagues were performed at a very early stage of the myelination to be able to capture sheath fully uncompacted and thus not thicker than 2-3 wraps. Furthermore, the serial sections performed were covering only few micrometers. The structure described in these studies may be explained by the fact that very early stages were imaged, at which they engulfed the axon in a relatively open structure of the non-compacted myelin sheath.

In later studies performed by Remahl and Hidelbrand using similar technique on CNS tissues no vermicular processes could be observed (Remahl and Hildebrand, 1990; Remahl and Hilderbrand, 1990) These observations were performed on tissues with a more advanced myelination state and based on large volumes of data and thus, the myelin conformation up to $212 \mu \mathrm{m}$ along the axon could be followed. In these studies the authors observed that the thickness of the myelin sheath was non-uniform along the growing sheath and that portion of non-compacted sheath would cover the axon at the lateral edges of the sheath. These observations led to the hypothesis that the "early glial sheaths of developing CNS axons actively elongate and undergo extensive remodeling before compaction" (Remahl and Hildebrand, 1990). These observations were not in line with the previous myelination models proposed by Bunge and colleagues: neither "the carpet crawler model" (Bunge et al., 1961; Hirano and Dembitzer, 1967), nor the pulsation mediated wrapping process proposed by Richard and colleagues (Richards et al., 1983). Indeed, in these models the oligodendrocyte processes would spread along the future myelin segments in a one wrap manner before continuing the spiral ensheathment. 
Our results confirm the observations and hypothesis made by Remahl and Hidelbrand concerning the lateral extension of the layers during the myelin sheath maturation however, our data also gives a deep mechanistic and molecular insight in this process, absent in the work of Remahl. Using live imaging in Zebrafish and correlating our observations with the TEM longitudinal investigations of mouse optic nerve over time, we could show the lateral extension of the successive layers towards the node of Ranvier (fig 4.2). Furthermore, we could precisely assess the positioning and the morphology of myelin layers along the growing sheath confirming the concept of non-uniform growing myelin sheath proposed in previous studies (Knobler et al., 1976; Remahl and Hildebrand, 1990; Sobottka et al., 2011). We also could model the myelin sheath's shape and its development during myelin biogenesis (fig 4.2).
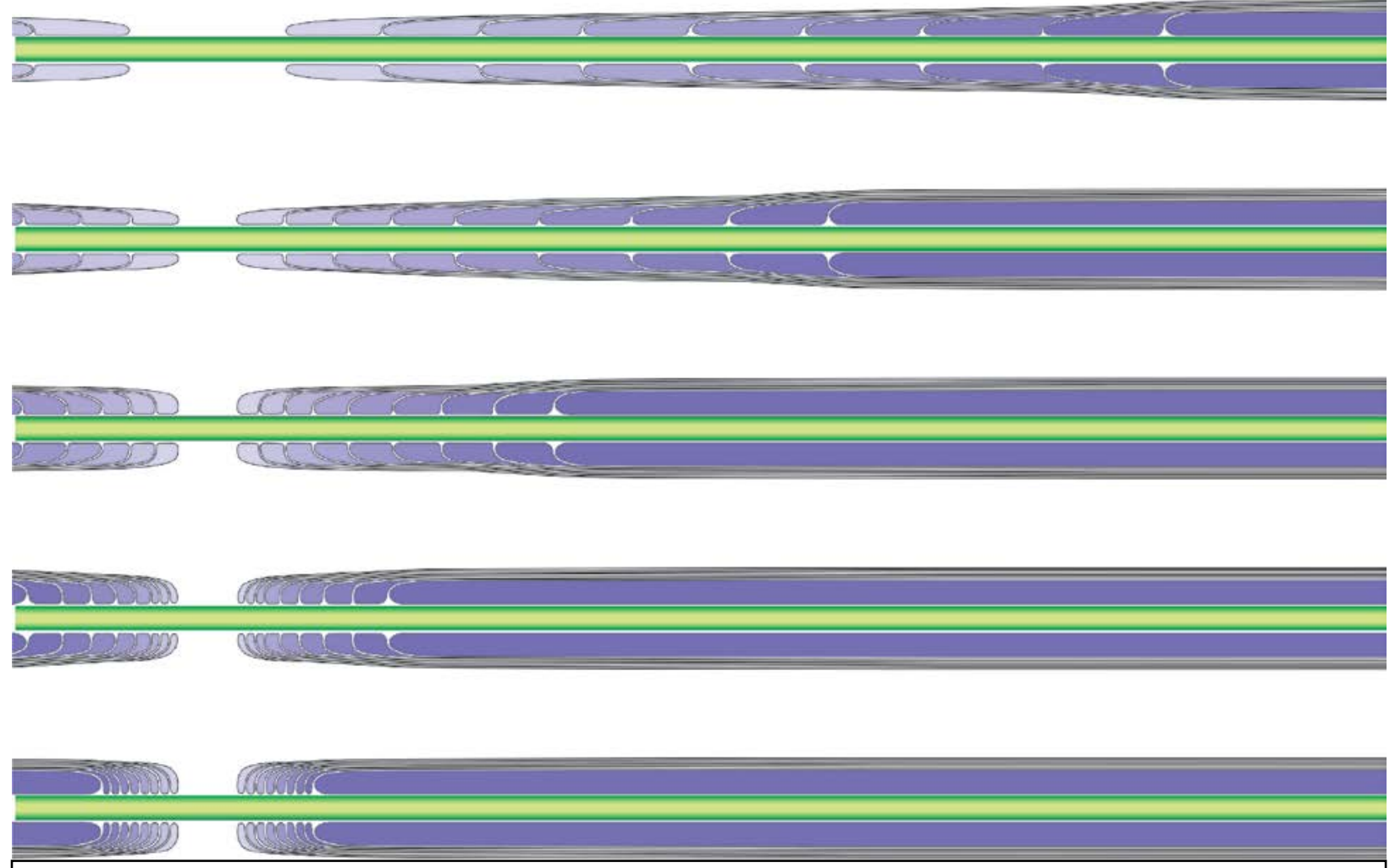

Figure 4.2: Lateral extension of the myelin layers during development.

The successive myelin layers extend laterally on the surface of the sheath until the zone where the node of Ranvier will form. The distance between the successive layers decrease when they reach the node, ultimately forming tightly apposed paranodal loops. 


\subsection{Identification of the myelin leading edge}

Understanding the sheath morphology during the growth gave us a better view on its development, however a critical question remained to be answered: where is the leading edge of the growing myelin sheath located? As mentioned previously, due to the large differences in scale magnitude needed to study the sheath morphology and considering the light diffraction limit using light microscopy, the direct live-imaging to identify the growth zone of the developing myelin sheath in vivo was not possible. Furthermore, electron microscopy can exclusively be performed on fixed tissues excluding any dynamic investigation.

To overcome these shortcomings we made use of a well established molecular tool to track the plasma membrane trafficking at a subcellular resolution: the Vesicular Stomatitis Virus (Farquhar, 1985); (Balch et al., 1994); (de Vries et al., 1998). Upon infection, the oligodendrocytes are producing a large amount of the VSV coat protein named VSV-G. The VSV$\mathrm{G}$ is transported by the biosynthetic secretory pathway of the cell and can thus be used to identify the site where the newly synthesized material is incorporated into the membrane. We found that the great majority of VSV-G would localize at the inner tongue of the myelin during the growth phase of the sheath, but would be mainly localized at the outer tongue once the myelin is mature. This would imply that the newly synthesized material is preferentially inserted at the inner tongue during the growth phase and that after the growth is completed the trafficking towards the inner tongue would be greatly reduced.

Bunge and colleagues already hypothesized that "the spiraling takes place by advance of the inner end of the spiral around the axon" (Bunge et al., 1961). Due to the one to one relationship between Schwann cell and axon in the PNS, as well as the motion of the cell nucleus around the axon during myelination (Pomerat et al., 1967) these early papers concluded that the cell would rotate around the axon or incorporate new material from the non-compacted outer wrap of the myelin to achieve the spiraled myelin (Geren and Schmitt, 1954). However, Webster, Bunge and colleagues postulated that in the CNS and PNS the inner mesaxon is the advancing tip of the myelin sheath (Webster, 1971), mainly due to the fact that Schwann cells would wrap the PNS axons without rotation of the cell body around the sheath (Bunge et al., 1989). These 
hypotheses were challenged in more recent studies with the visualization of coils of cytoplasm around the growing axon in the CNS and PNS that led to alternative myelination models where the coiling processes or the outer tongue were described as areas where the material would be added to the myelin (Berry et al., 1995); (Pedraza et al., 2009); (loannidou et al., 2012; Sobottka et al., 2011). Our observations are in line with some of the earlier proposed hypothesis, stating that the advancing of the inner tongue is responsible for the wrapping.

\subsection{Central regulators of the myelin growth}

Knowing that the plasma membrane trafficking is directed towards the inner tongue during the growth we also addressed the molecular mechanisms responsible for the polarized growth. The PI3-kinase activation and accumulation of its lipid product PI(3,4,5)P3 have been described to play a central role in cellular polarity by recruiting Rho GTPases to the leading edge (Ridley et al 2010). Furthermore, the PI3K/Akt/mTOR pathway was also described to be involved in myelin growth (Flores et al., 2008); (Goebbels et al., 2010).

Using immuno-fluorescence and immuno-electron microscopy in cell culture and mice assays we could identify several components of the PI3K/Akt/mTOR pathway as well as filamentous actin to be enriches at the growth zone: the inner tongue in vivo and the outer rim in vitro. Furthermore, we could correlate the abundance of these markers with the size of the noncompacted area at the growth zone. Indeed, the cytoplasmic area at the leading edge is larger during the growth phase with a more intense Phospho-Akt and F-actin labeling than in mature cells.

We went further to test these correlations by using the PTEN mutant described by Sandra Goebbles and colleagues. In these mutants the amount of PIP3 and downstream effectors were upregulated and a drastic increase in myelin thickness was described (Goebbels et al., 2010; Goebbels et al., 2012). We confirmed these observations and found, in addition, that the inner tongue of myelin optic nerve was significantly larger in these mutants at P23 and P60 as compared to wild type. This data suggests that the increase in PIP3 and its downstream effectors caused by the PTEN inactivation is responsible for the activation and the polarization 
of the inner tongue at the leading edge, eventually leading to a hyper-myelination and enlarged inner tongue.

The localization of the F-actin at the growth zone is a common feature for most of the cell types (Hall 1998) and its regulation by the Rho GTPases is well known (Nobes and Hall, 1995); (Ridley et al., 1992). Indeed, the Rac1 and CDC42 are involved in the polarization and stabilization of the lamelipodia at the cell periphery (Nobes and Hall, 1995); (Machesky and Hall, 1997). It has been described by Thurnherr and colleagues that in oligodendrocytes of the knockout mouse mutants for Rac1 and CDC42 the myelin sheath would be thinner and that the inner tongue would be greatly enlarged (Thurnherr et al., 2006).

Our findings (plasma membrane trafficking is directed towards the inner tongue and that the amount of F-actin at the inner tongue correlates with the growth state of the sheath) and the observations done by Thurnherr et al would imply that the lack of Rac1 and CDC42 would impair the myelin growth by altering the f-actin cytoskeleton. It is possible that the f-actin cytoskeleton is directing the growth at the leading edge guiding and facilitating vesicles fusion at the inner tongue. Indeed, in these mutants an accumulation of vesicular profiles has been described at the inner tongue in addition to a thinner myelin, suggesting that the functionality of the upstream components of the plasma membrane trafficking machinery is not altered by these mutations and that the material is still normally transported to the growth zone but not well incorporated.

\subsection{The cytoplasmic channels: a direct track from the cell body to the leading edge}

When identifying the growth zone at the inner tongue of the myelin sheath during development in wild type and in the PTEN mutant mice, the question of how the material is brought to the growth zone through the entire compacted myelin sheath arose. If we consider a sheath of $100 \mu \mathrm{m}$ long with 6 wraps during the intense phase of growth, a massive amount of vesicles would have to be transported through the outer tongue, the forming paranodal loops and the inner tongue to reach the incorporation site. It seems too energy consuming for the cell to use this way of transport, especially knowing that in the PNS myelin-forming cells the Schmitt 
Lantermann incisures are providing a direct link between the cell body and the inner tongue (Webster, 1971).

Using high pressure frozen optic nerves at different time points of myelin development we could identify by TEM a large amount of cytoplasmic inclusions within the compacted sheath. Cytoplasmic inclusions in CNS compact myelin have rarely been described due to their very transient appearance and were referred as "isolated island of cytoplasm" (Hirano and Dembitzer, 1967). These cytoplasmic channels present a similar morphology to the Schmitt Lantermann incisures and appeared to be only a transient feature of the developing myelin sheath in the optic nerve and corpus callosum. Furthermore, within these cytoplasmic channels we could identify microtubules as well as a great amount of vesicular patterns that would disappear with the sheath maturation. Considering these features and especially their correlation with the growth of the sheath we proposed that such cytoplasmic channels are shortcuts from the cell body to the growth zone at the inner tongue.

In recent studies the plasticity of the myelin sheath has been investigated in adult mice, notably in social isolation and enrichment (Liu et al., 2012), (Makinodan et al., 2012; Yang et al., 2013) leading to the concept of adult myelin plasticity. In these studies the deprivation of social experience influenced negatively myelination in some cognitive areas possibly being responsible for cognition impairment (Liu et al., 2012); (Makinodan et al., 2012). Enriched environment, in contrast, has a positive effect on myelin formation during development (Yang et al., 2013).

Thus, we wanted to test whether the cytoplasmic channels would re-appear in the adult myelin by experimental reactivation myelination once the developmental program has been terminated. Using this genetic model we found that the channels re-open prior to myelin thickening in response to the increase of PIP3 levels.

Considering the myelin plasticity in adult we now hypothesize that these channels also have a role beyond development and their potential to re-open under physiological conditions involving a transport through the myelin sheath to the axon for myelin remodeling and axonal metabolic support. Furthermore, considering their morphology being very similar to the Schmitt Lantermann incisures in the PNS, it is possible that in thicker and longer CNS myelin 
sheaths, such as the ones found in the spinal cord, the cytoplasmic channels might be more abundant even in the adult.

In summary we propose that these channels form a dynamic cytoplasmic network with the outer and inner tongue, but the question of how the cytoplasmic channels are regulated remains to be answered. Due to the presence of microtubules and the fact that the channels are responsive to the PI3 kinase pathway, one possibility is that the local regulation of different cytoskeleton elements (actin or/and microtubules) and, on the other side, the zipping force of the MBP molecules and are possible regulators of the opening and closing of the channels.

\subsection{Forming myelin outfoldings during normal development}

The data sets acquired with the FIBSEM allowed us to visualize the cytoplasmic channels along the forming myelin sheath, which shows that the cytoplasmic channels end within myelin outfoldings. These outfoldings are found in sheaths with various thicknesses and contain large amount of myelin membrane.

The number of outfoldings decreased drastically with the maturation of the myelin sheath as agreeing with the reported observations in amphibian cerebellum (Rosenbluth, 1966).

We proposed that the outfoldings are formed by preferential delivery of membrane through the cytoplasmic channels within the compact sheath and into the inner tongue leading to a focal delivery and incorporation of massive amounts of plasma membrane components. Since the lateral growth of the layers is limited by the position of the successive superficial layers, is it possible that the rate at which the material is added to the innermost layer is larger than the growth rate of the successive layers. Due to the structure of myelin this would result in a vertical expansion and the subsequent formation of an outfolding (fig 4.3).

In CDC42 and Rac1 mutants the amount of outfoldings was shown to be significantly larger than in wild type mice (Thurnherr et al., 2006). The authors propose that CDC42 and Rac1, which are involved in the function of the cytoskeleton, perturb the lateral extension of the layers leading to outfolding. Furthermore, in PTEN mutants it have also been shown to have an increased number of outfoldings (Goebbels et al., 2010; Goebbels et al., 2012). 
A

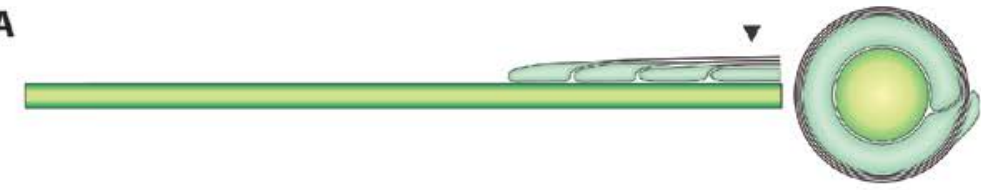

B

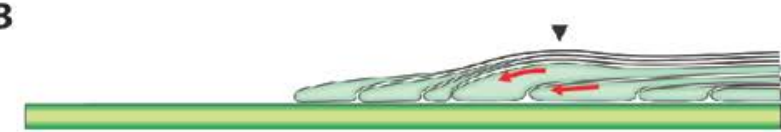

C

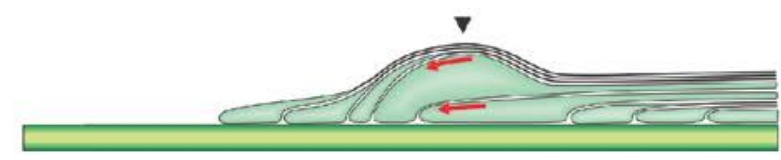

D

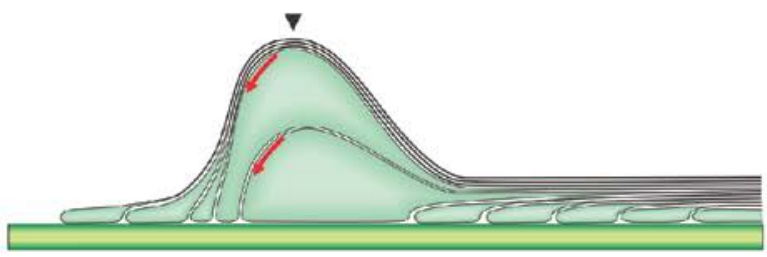

E

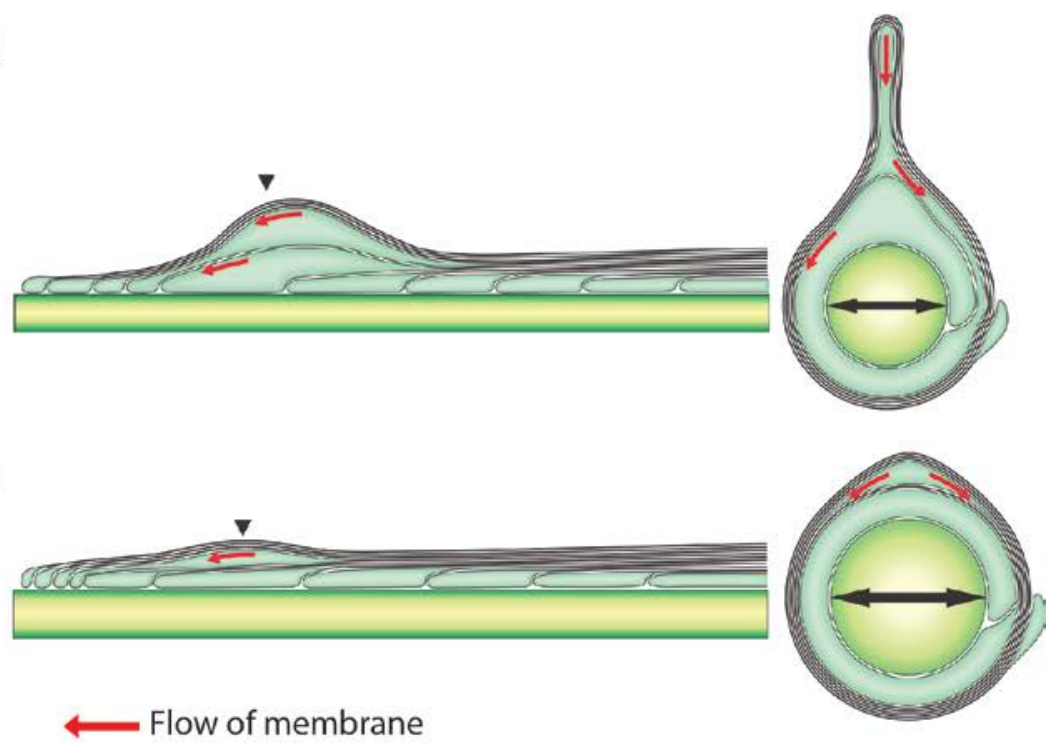

$\mathbf{F}$

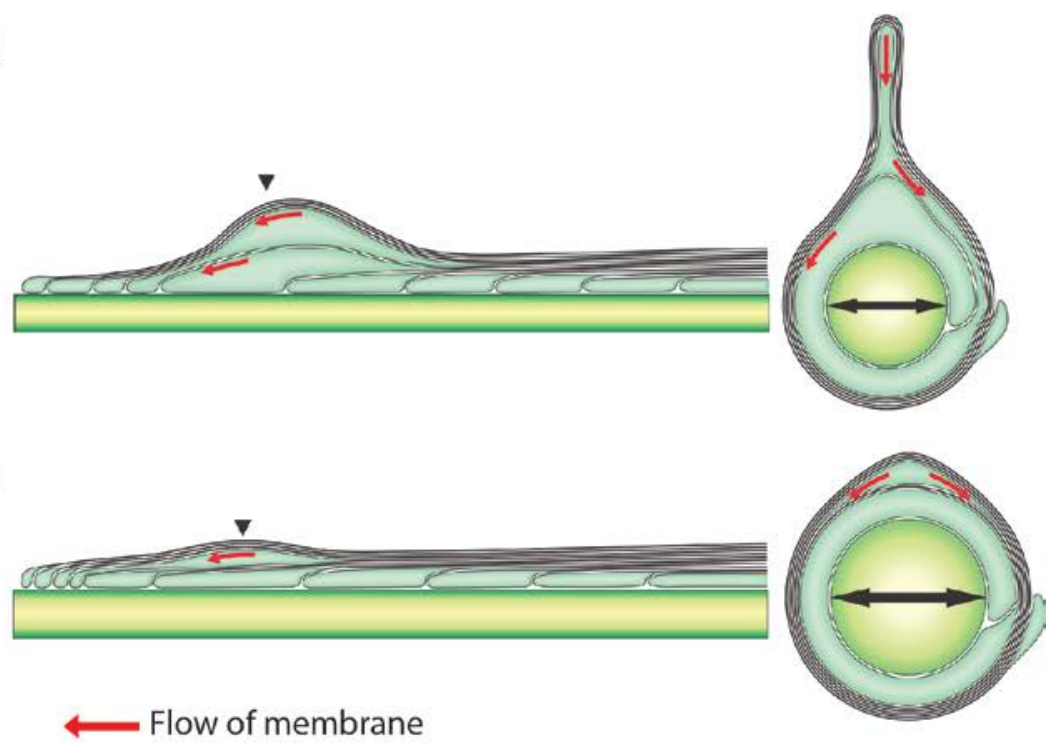

Flow of membrane
Figure 4.3: Model of outfolding formation and resolution during myelin growth.

A) Growing myelin sheath after the few first wraps of the oligodendrocyte process. B) Cytoplasmic channels bring to lateral edges of the fourth and fifth layers a massive amount of newly synthesized material. C) Due to the large amount of material to be incorporated and the presence of additional superficial wraps not allowing the further lateral extension the material is accumulated in a vertical manner. D) The vertical extension of the layers continues until the cytoplasmic channels are closed. E, F) The stored material within the outfolding is redistributed to complete the lateral extension of the layers and to accommodate the axonal diameter increase. 
This is possibly due to the constant incorporation of material at the inner tongue before the myelin layers are fully stabilized by the radial component that limits the gliding of the layers on each other.

These conclusions are in line with our observations, which show that the outfoldings are caused by a dysregulation of the balance between layer growth and material input. Our data shows that this can happen during development due to the massive focal incorporation of material or in pathological conditions by reduction of the speed at which the layers extend laterally (Pereira et al 2012).

We find that outfoldings and cytoplasmic channels are disappearing at the same time in development. Once the cytoplasmic channels are closed the outfoldings are likely to stop receiving additional material. However, this happens at a time when the sheath is not fully mature and when the diameter of the axon is likely to grow further, leading to a large membrane requirement for the myelin sheath to keep an optimal G-ratio (Rushton, 1951); (Chomiak and $\mathrm{Hu}, 2009$ ). The additional material needed for this myelin thickening is possibly taken from the large myelin reservoirs of the outfoldings. To reinforce this hypothesis we could confirm that the radial component of the myelin sheath, which stabilizes the layers of the sheath with each other (Debruin and Harauz, 2007), is not present at the time of the outfolding formation, which is a prerequisite for the myelin layers to glide on each other.

\subsection{Sheath compaction proceeds from the superficial myelin layers}

In addition to the cytoplasmic channels, we could observe in a large amount of P10 optic nerve sheaths, myelin layers that would be fully uncompacted, but still closely aligned and be apart from each other by only a $13 \mathrm{~nm}$ space. These layers were found to be located almost invariably from the inner tongue of the growing sheath, leading to uncompacted wraps that could make from 2 up to 8 layers of the sheath. We found that within the uncompacted layers the level of MBP protein is drastically reduced, so that the critical concentration required to bind the two opposite membranes is possibly not reached.

The observed gradient of MBP molecules being high in the outer and low in the inner layers of the growing sheath is against the notion that MBP mRNA granules is translated upon axonal 
signal at the inner mesaxon (Ainger et al., 1993; Colman et al., 1982; Trapp et al., 1987), (Wake et al 2011). We find that the free ribosomes are equally distributed in all the uncompacted areas of the myelin sheath allowing the MBP protein to be made in the entire cytoplasmic network of the sheath. Axonal signals could be transmitted to increase the translation of MBP mRNA in order to avoid premature compaction of glial processes presenting a similar diameter to the axons. We also confirm previously published data showing that the compaction of the sheath starts rapidly after the first engulfment of the axon by the myelin sheath (Hildebrand et al., 1993), and thus compacted layers can be found in 2-3 wraps thick myelin. Furthermore, once MBP is bound to the opposite membranes it polymerizes into a network, creating a nucleation point from which the MBP grows further and the compaction is initiated (Aggarwal et al., 2013). Due to the polarized growth at the inner tongue of the sheath it is likely than the speed of the growth is faster than the compaction by MBP. A similar idea was proposed by Wiggins and colleagues based on few growing myelin sheaths that could not be explained by the myelination models proposed at that time (Wiggins et al., 1988).

Knowing that the first nucleation points are formed by MBP at a 2-3 wrap stage, the compaction progresses subsequently from the most external layers towards the inside of the sheath (in an out-to-in motion).

The morphological analysis carried out on the fully uncompacted layers showed a very reproducible spacing, implying that another protein complex maintains the membranes in close contact. Due to the very large cytoplasmic domain and the great abundance of the CNP in the CNS myelin sheath, we investigated its distribution and found that it was localized in these uncompacted layers. Furthermore, it has been shown that the overexpression of the CNP in oligodendrocytes would lead to the opening of the major dense line of the sheath thereby increasing the amount of uncompacted layers (Gravel et al., 1996). However, the uncompacted layers would appear at a more unpredictable location within the sheath, while our results show that they are found invariably at the inner layers of the sheath.

There is an evidence that CNP, in addition to being linked to actin and microtubules (De Angelis and Braun, 1996), (Bifulco et al., 2002), is also able to dimerise generating a spacer of $10-15 \mathrm{~nm}$ 
between the opposite membranes, which is in the perfect accordance with the spacing of the uncompacted layers observed in the P10 optic nerves.

Using knockout mice mutants for MBP and CNP we proposed that there is a "tug of war" between the zipping force of MBP and the CNP spacing during myelin development. Indeed, when reducing the amount of MBP we would see more uncompacted layers within the sheath, whereas the reduction of CNP resulted in an almost complete abolition of uncompacted layers during the growth phase.

The balance between compacted and uncompacted layers, as well as the direction of the zipping, is a prerequisite for an optimal compaction of the myelin sheath. In addition, it is likely needed to prevent the formation of multiple nucleation sites that would lead to islands of cytoplasm unlinked to any mesaxon.

Furthermore, this modality of compaction also allows the spatial separation of the growth and compaction of the myelin sheath (fig 4.4).

A
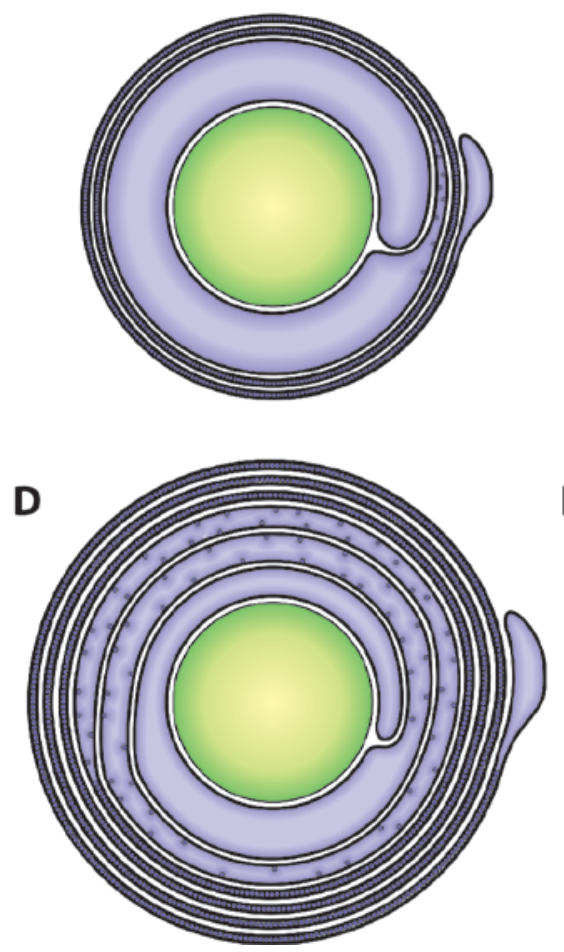

B
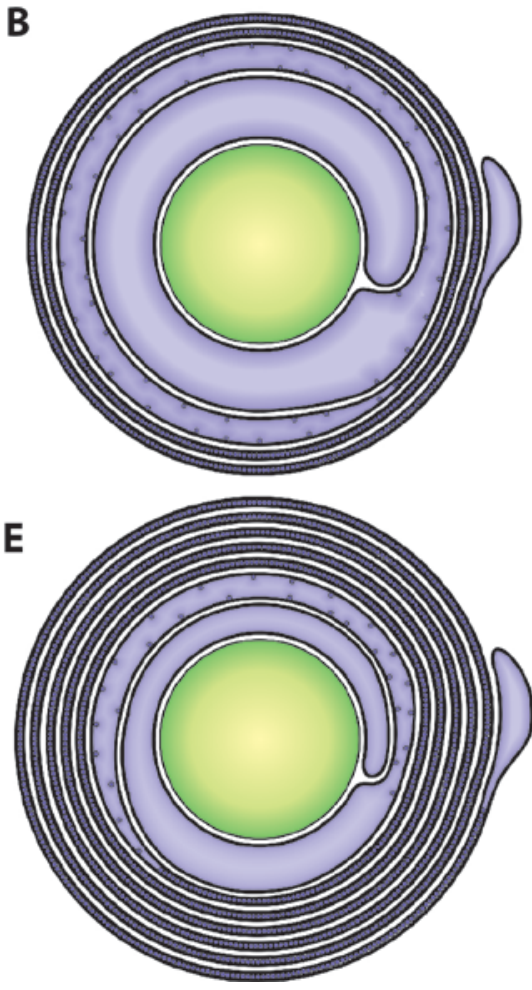
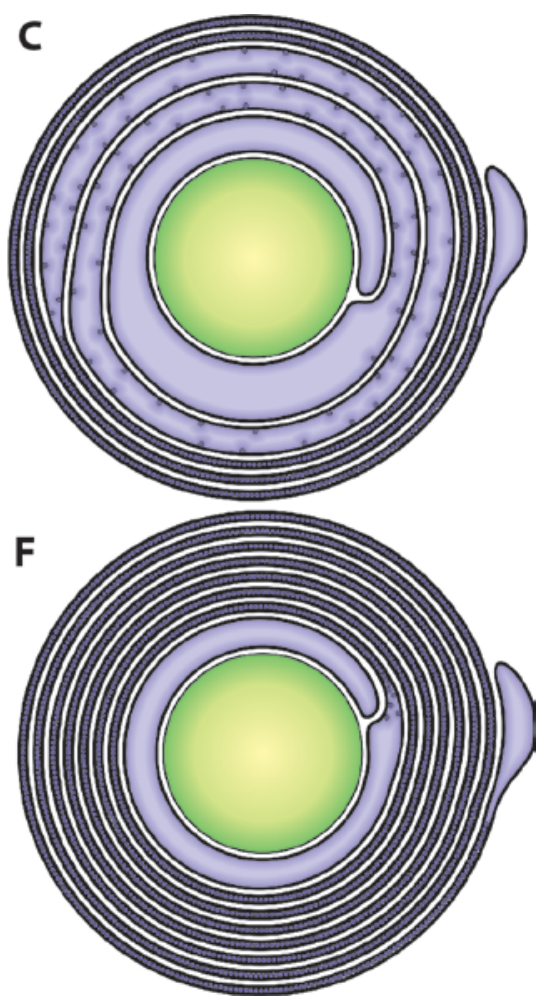
Figure 4.4: Model of the myelin morphology during growth and MBP compaction.

A-F) Model of the evolution of the sheath compaction during myelin growth in a cross sectional view. A) Early time point of the wrapping showing a thin myelin sheath partially or totally compacted. B-D) The growth speed of the inner tongue is faster than the compaction by MBP leading to a gradient of compaction within the sheath and leaving the inner most layers uncompacted. E,F) Once the growth speed is slowing down the compaction by MBP is progressively zipping together the remaining uncompacted layers from the outer most layers towards the inner layers.

\subsection{Axo-glia communication during myelination process}

The interaction between the myelin producing cells and the axons during the developmental myelination is a very complex communication that is only poorly understood in the CNS. It is well known that the axon caliber is a major signal for the myelinating cells to initiate the wrapping. Indeed, only axons thicker than 1 micrometer of diameter are myelinated in the PNS ((Sherman and Brophy, 2005)), whereas axons with a diameter of at least $300 \mathrm{~nm}$ are myelinated by oligodendrocytes in the CNS ((Almeida et al., 2011; Lee et al., 2012)). The diameter of the axon itself gives mechanical cues for the wrapping, but it is not sufficient and enough to reach proper myelination. Indeed, several adhesion and signaling molecules at the surface of the axon have been described to be essential for initiating the wrapping and for regulating the myelin thickness. Among them are neuregulins (Birchmeier and Nave, 2008), neurotrophins (Rosenberg et al., 2006), neural cell adhesion L1 ((Nave and Trapp, 2008), (Coman et al., 2005)), laminins (Laursen et al., 2009), integrins and contactins (Charles et al., 2002).

In addition to these complex molecular communications the electrical activity of the axon has been described to be involved in the myelination process and could be one of the most important signals that would allow the myelinating cells to differentiate between axons and other processes. The electrical activity of the axons has been shown to regulate myelination in vivo and in vitro (Gyllensten and Malmfors, 1963), (Tauber et al., 1980), (Demerens et al., 1996). In this context it is interesting to note that, according to our observations the oligodendrocytes responded to electrical currents by polarizing the PI3-kinase pathway towards the cathode leading to the polarization of membrane trafficking. It is possible that the oligodendrocyte processes are directed towards the active axons due to the electrical 
fluctuation at their surface by polarization of the PI3-kinase pathway. Furthermore, during myelination the clustering of the ion channels by lateral extension of the most superficial myelin layer may play an important role in the intensification of the local currents at the surface of the axon from the edges of the growing sheath. This would trigger a lateral electrotaxis for the deeper and shorter layers facilitating their lateral extension. However, the molecules responsible to sense the electrical activities in the myelin sheath are not yet known.

In conclusion, this work sheds new light onto the wrapping processes in the central nervous system by bringing together fundamental knowledge on the development of the myelin sheath morphology during its formation, on the trafficking of material towards the growth front and on molecular mechanisms responsible for the polarized growth.

These multiple aspects of the myelination process may not only help bringing clues to understand how myelin is formed in development, but also on abnormal structures formed in pathology, such as enlarged inner tongues, outfoldings or defaults in the lateral migration of the layers. 


\section{Summary}

Based on the work of this thesis and previously published studies we can now propose the following "working model" of myelin biogenesis. First, the oligodendrocytic process contacts the axon and, upon sensing the electrical activity of the axon or recognizing molecules expressed at the surface of the axon, the process flattens onto the surface of the axon and covers a certain length of the axon with one wrap. The oligodendrocyte plasma membrane trafficking machinery is then polarized towards this process, leading to the transport of membrane component to the advancing tip via the pathway dependent on PI3K signaling. Subsequently the sheath starts to advance under the first wrap at the center of the segment in front of the oligodendrocyte process. The newly synthesized material is preferentially brought to the inner tongue, enabling the winding of the oligodendrocytic process around the axon underneath the previously deposited wraps. This process leads to the radial growth of the myelin sheath. Together with this motion the edges of the layers extend laterally along the axon, leading to a triangular shaped sheath with the base of the triangle being the outer tongue and the tip being the inner most layers of the sheath at the center of the segment. After few wraps, the cytoplasmic leaflets of the myelin membrane start to be compacted by MBP at nucleation sites in the outer layers of the myelin sheath. Due to the polarized growth of the sheath from the inner tongue and depending on the speed of the growth and the amount of MBP molecules a gradient of compaction can be observed in these growing myelin segments with the inner most wraps remaining uncompacted. However, even in the outer most layers that became compacted some cytoplasmic channels remain open and run through the compacted myelin sheath, allowing the direct delivery to the inner tongue at one or several growth sites. The opening of these channels, as well as the growth of the sheath is regulated by the PI3P levels. Due to the compaction of the sheath the delivery of the vesicles is directed to few areas by the cytoplasmic channels at the inner layers along the internode that may lead to the formation of membrane reservoirs in the shape of myelin outfoldings.

Considering the weak interactions between the extracellular leaflets of the myelin membranes and the absence of mature radial components these layers may glide on each other at the 
extracellular sides. This allows the addition of new layers from the inner tongue but also the increase in diameter of the underlying axon that occurs after myelination. At this point the membrane stored in the outfoldings is likely to be used to accommodate this diameter increase without losing myelin thickness.

When the myelin reaches its optimal thickness according the G-ratio, membrane trafficking is slowed down leading to the closing of cytoplasmic channels. Next, the more superficial myelin layers cover the entire length of the internode and become connected by their lateral edges to the axon in the nodal region with septate junctions. The underlying layers that are progressively smaller will finish their lateral extension with time leading to tightly apposed paranodal loops.

Finally, after all successive layers reach the paranodal region, the myelin thickness is constant along the entire internode and the compaction of the layers is complete, leaving only the inner and outer tongue uncompacted and the radial components stabilizing the overall myelin structure. 


\section{References}

Aggarwal, S., Snaidero, N., Pahler, G., Frey, S., Sanchez, P., Zweckstetter, M., Janshoff, A., Schneider, A., Weil, M.T., Schaap, I.A., et al. (2013). Myelin membrane assembly is driven by a phase transition of myelin basic proteins into a cohesive protein meshwork. PLoS Biol 11, e1001577.

Aggarwal, S., Yurlova, L., Snaidero, N., Reetz, C., Frey, S., Zimmermann, J., Pahler, G., Janshoff, A., Friedrichs, J., Muller, D.J., et al. (2011). A size barrier limits protein diffusion at the cell surface to generate lipid-rich myelin-membrane sheets. Dev Cell 21, 445-456.

Ainger, K., Avossa, D., Morgan, F., Hill, S.J., Barry, C., Barbarese, E., and Carson, J.H. (1993). Transport and localization of exogenous myelin basic protein mRNA microinjected into oligodendrocytes. J Cell Biol $123,431-441$.

Allen, N.J., and Barres, B.A. (2009). Neuroscience: Glia - more than just brain glue. Nature 457, 675-677.

Allt, G. (1969). Ultrastructural features of the immature peripheral nerve. J Anat 105, 283-293.

Almeida, R.G., Czopka, T., Ffrench-Constant, C., and Lyons, D.A. (2011). Individual axons regulate the myelinating potential of single oligodendrocytes in vivo. Development 138, 4443-4450.

Arimura, N., and Kaibuchi, K. (2007). Neuronal polarity: from extracellular signals to intracellular mechanisms. Nat Rev Neurosci 8, 194-205.

Bakhti, M., Snaidero, N., Schneider, D., Aggarwal, S., Mobius, W., Janshoff, A., Eckhardt, M., Nave, K.A., and Simons, M. (2013). Loss of electrostatic cell-surface repulsion mediates myelin membrane adhesion and compaction in the central nervous system. Proc Natl Acad Sci U S A 110, 3143-3148.

Balch, W.E., McCaffery, J.M., Plutner, H., and Farquhar, M.G. (1994). Vesicular stomatitis virus glycoprotein is sorted and concentrated during export from the endoplasmic reticulum. Cell 76, 841852.

Bauer, N.G., and ffrench-Constant, C. (2009). Physical forces in myelination and repair: a question of balance? Journal of biology 8,78 .

Baumann, N., and Pham-Dinh, D. (2001). Biology of oligodendrocyte and myelin in the mammalian central nervous system. Physiol Rev 81, 871-927.

Berry, M., Ibrahim, M., Carlile, J., Ruge, F., Duncan, A., and Butt, A.M. (1995). Axon-glial relationships in the anterior medullary velum of the adult rat. J Neurocytol 24, 965-983.

Bifulco, M., Laezza, C., Stingo, S., and Wolff, J. (2002). 2',3'-Cyclic nucleotide 3'-phosphodiesterase: a membrane-bound, microtubule-associated protein and membrane anchor for tubulin. Proc Natl Acad Sci U S A 99, 1807-1812.

Birchmeier, C., and Nave, K.A. (2008). Neuregulin-1, a key axonal signal that drives Schwann cell growth and differentiation. Glia 56, 1491-1497. 
Buckley, C.E., Goldsmith, P., and Franklin, R.J. (2008). Zebrafish myelination: a transparent model for remyelination? Disease models \& mechanisms 1, 221-228.

Bunge, M.B., Bunge, R.P., and Ris, H. (1961). Ultrastructural study of remyelination in an experimental lesion in adult cat spinal cord. J Biophys Biochem Cytol 10, 67-94.

Bunge, R.P. (1968). Glial cells and the central myelin sheath. Physiol Rev 48, 197-251.

Bunge, R.P., Bunge, M.B., and Bates, M. (1989). Movements of the Schwann cell nucleus implicate progression of the inner (axon-related) Schwann cell process during myelination. J Cell Biol 109, 273284.

Charles, P., Reynolds, R., Seilhean, D., Rougon, G., Aigrot, M.S., Niezgoda, A., Zalc, B., and Lubetzki, C. (2002). Re-expression of PSA-NCAM by demyelinated axons: an inhibitor of remyelination in multiple sclerosis? Brain 125, 1972-1979.

Chomiak, T., and Hu, B. (2009). What is the optimal value of the g-ratio for myelinated fibers in the rat CNS? A theoretical approach. PLoS One 4, e7754.

Colman, D.R., Kreibich, G., Frey, A.B., and Sabatini, D.D. (1982). Synthesis and incorporation of myelin polypeptides into CNS myelin. J Cell Biol 95, 598-608.

Coman, I., Barbin, G., Charles, P., Zalc, B., and Lubetzki, C. (2005). Axonal signals in central nervous system myelination, demyelination and remyelination. J Neurol Sci 233, 67-71.

Czopka, T., and Lyons, D.A. Dissecting mechanisms of myelinated axon formation using zebrafish. Methods Cell Biol 105, 25-62.

De Angelis, D.A., and Braun, P.E. (1996). 2',3'-Cyclic nucleotide 3'-phosphodiesterase binds to actinbased cytoskeletal elements in an isoprenylation-independent manner. J Neurochem 67, 943-951.

De Robertis, E., Gerschenfeld, H.M., and Wald, F. (1958). Cellular mechanism of myelination in the central nervous system. The Journal of biophysical and biochemical cytology 4, 651-656.

de Vries, H., de Jonge, J.C., Schrage, C., van der Haar, M.E., and Hoekstra, D. (1997). Differential and cell development-dependent localization of myelin mRNAs in oligodendrocytes. J Neurosci Res 47, 479-488.

de Vries, H., Schrage, C., and Hoekstra, D. (1998). An apical-type trafficking pathway is present in cultured oligodendrocytes but the sphingolipid-enriched myelin membrane is the target of a basolateraltype pathway. Mol Biol Cell 9, 599-609.

Debruin, L.S., and Harauz, G. (2007). White matter rafting--membrane microdomains in myelin. Neurochem Res 32, 213-228.

Demerens, C., Stankoff, B., Logak, M., Anglade, P., Allinquant, B., Couraud, F., Zalc, B., and Lubetzki, C. (1996). Induction of myelination in the central nervous system by electrical activity. Proc Natl Acad Sci U S A 93, 9887-9892.

Engstrom, H., and Wersall, J. (1958). Myelin sheath structure in nerve fibre demyelinization and branching regions. Exp Cell Res 14, 414-425.

Farquhar, M.G. (1985). Progress in unraveling pathways of Golgi traffic. Annu Rev Cell Biol 1, 447-488. 
Fayard, E., Moncayo, G., Hemmings, B.A., and Hollander, G.A. (2010). Phosphatidylinositol 3-kinase signaling in thymocytes: the need for stringent control. Science signaling 3 , re5.

Flores, A.I., Narayanan, S.P., Morse, E.N., Shick, H.E., Yin, X., Kidd, G., Avila, R.L., Kirschner, D.A., and Macklin, W.B. (2008). Constitutively active Akt induces enhanced myelination in the CNS. J Neurosci 28, 7174-7183.

Friede, R.L., and Miyagishi, T. (1972). Adjustment of the myelin sheath to changes in axon caliber. The Anatomical record 172, 1-14.

Funfschilling, U., Supplie, L.M., Mahad, D., Boretius, S., Saab, A.S., Edgar, J., Brinkmann, B.G., Kassmann, C.M., Tzvetanova, I.D., Mobius, W., et al. (2012). Glycolytic oligodendrocytes maintain myelin and longterm axonal integrity. Nature 485, 517-521.

Geren, B.B., and Schmitt, F.O. (1954). The Structure of the Schwann Cell and Its Relation to the Axon in Certain Invertebrate Nerve Fibers. Proc Natl Acad Sci U S A 40, 863-870.

Goebbels, S., Oltrogge, J.H., Kemper, R., Heilmann, I., Bormuth, I., Wolfer, S., Wichert, S.P., Mobius, W., Liu, X., Lappe-Siefke, C., et al. Elevated phosphatidylinositol 3,4,5-trisphosphate in glia triggers cellautonomous membrane wrapping and myelination. J Neurosci 30, 8953-8964.

Goebbels, S., Oltrogge, J.H., Kemper, R., Heilmann, I., Bormuth, I., Wolfer, S., Wichert, S.P., Mobius, W., Liu, X., Lappe-Siefke, C., et al. (2010). Elevated phosphatidylinositol 3,4,5-trisphosphate in glia triggers cell-autonomous membrane wrapping and myelination. J Neurosci 30, 8953-8964.

Goebbels, S., Oltrogge, J.H., Wolfer, S., Wieser, G.L., Nientiedt, T., Pieper, A., Ruhwedel, T., Groszer, M., Sereda, M.W., and Nave, K.A. (2012). Genetic disruption of Pten in a novel mouse model of tomaculous neuropathy. EMBO Mol Med 4, 486-499.

Graeber, M.B., Li, W., and Rodriguez, M.L. (2011). Role of microglia in CNS inflammation. FEBS Lett 585, 3798-3805.

Gravel, M., Peterson, J., Yong, V.W., Kottis, V., Trapp, B., and Braun, P.E. (1996). Overexpression of 2',3'cyclic nucleotide 3'-phosphodiesterase in transgenic mice alters oligodendrocyte development and produces aberrant myelination. Mol Cell Neurosci 7, 453-466.

Griffiths, I., Klugmann, M., Anderson, T., Yool, D., Thomson, C., Schwab, M.H., Schneider, A., Zimmermann, F., McCulloch, M., Nadon, N., et al. (1998). Axonal swellings and degeneration in mice lacking the major proteolipid of myelin. Science 280, 1610-1613.

Gyllensten, L., and Malmfors, T. (1963). Myelinization of the optic nerve and its dependence on visual function--a quantitative investigation in mice. Journal of embryology and experimental morphology 11 , 255-266.

Hall, A. (1998). Rho GTPases and the actin cytoskeleton. Science 279, 509-514.

Hartline, D.K., and Colman, D.R. (2007). Rapid conduction and the evolution of giant axons and myelinated fibers. Current biology : CB 17, R29-35.

Hildebrand, C., Remahl, S., Persson, H., and Bjartmar, C. (1993). Myelinated nerve fibres in the CNS. Prog Neurobiol 40, 319-384. 
Hirano, A., and Dembitzer, H.M. (1967). A structural analysis of the myelin sheath in the central nervous system. J Cell Biol 34, 555-567.

Hirano, A., Dembitzer, H.M., Becker, N.H., Levine, S., and Zimmerman, H.M. (1970). Fine structural alterations of the blood-brain barrier in experimental allergic encephalomyelitis. J Neuropathol Exp Neurol 29, 432-440.

Ioannidou, K., Anderson, K.I., Strachan, D., Edgar, J.M., and Barnett, S.C. (2012). Time-lapse imaging of the dynamics of CNS glial-axonal interactions in vitro and ex vivo. PLoS One 7, e30775.

Jaros, E., and Jenkison, M. (1983). Quantitative studies of the abnormal axon-Schwann cell relationship in the peripheral motor and sensory nerves of the dystrophic mouse. Brain Res 258, 181-196.

Jessen, K.R., and Mirsky, R. (2005). The origin and development of glial cells in peripheral nerves. Nat Rev Neurosci 6, 671-682.

Kandel, E. R., J. H. Schwartz, and T. M. Jessell (2000). Principles of neural science. New York: McGrawHill, Health Professions Division.

Kirby, B.B., Takada, N., Latimer, A.J., Shin, J., Carney, T.J., Kelsh, R.N., and Appel, B. (2006). In vivo timelapse imaging shows dynamic oligodendrocyte progenitor behavior during zebrafish development. Nat Neurosci 9, 1506-1511.

Knobler, R.L., Stempak, J.G., and Laurencin, M. (1974). Oligodendroglial ensheathment of axons during myelination in the developing rat central nervous system. A serial section electron microscopical sttudy. J Ultrastruct Res 49, 34-49.

Knobler, R.L., Stempak, J.G., and Laurencin, M. (1976). Nonuniformity of the oligodendroglial ensheathment of axons during myelination in the developing rat central nervous system. A serial section electron microscopical study. J Ultrastruct Res 55, 417-432.

Laplante, M., and Sabatini, D.M. (2009). An emerging role of mTOR in lipid biosynthesis. Curr Biol 19, R1046-1052.

Lappe-Siefke, C., Goebbels, S., Gravel, M., Nicksch, E., Lee, J., Braun, P.E., Griffiths, I.R., and Nave, K.A. (2003). Disruption of Cnp1 uncouples oligodendroglial functions in axonal support and myelination. Nat Genet 33, 366-374.

Laursen, L.S., Chan, C.W., and ffrench-Constant, C. (2009). An integrin-contactin complex regulates CNS myelination by differential Fyn phosphorylation. J Neurosci 29, 9174-9185.

Laursen, L.S., Chan, C.W., and Ffrench-Constant, C. (2011). Translation of myelin basic protein mRNA in oligodendrocytes is regulated by integrin activation and hnRNP-K. J Cell Biol 192, 797-811.

Lee, S., Leach, M.K., Redmond, S.A., Chong, S.Y., Mellon, S.H., Tuck, S.J., Feng, Z.Q., Corey, J.M., and Chan, J.R. (2012). A culture system to study oligodendrocyte myelination processes using engineered nanofibers. Nat Methods 9, 917-922.

Levine, J.M., Reynolds, R., and Fawcett, J.W. (2001). The oligodendrocyte precursor cell in health and disease. Trends Neurosci 24, 39-47. 
Liu, J., Dietz, K., DeLoyht, J.M., Pedre, X., Kelkar, D., Kaur, J., Vialou, V., Lobo, M.K., Dietz, D.M., Nestler, E.J., et al. (2012). Impaired adult myelination in the prefrontal cortex of socially isolated mice. Nat Neurosci 15, 1621-1623.

Luse, S.A. (1956). Formation of myelin in the central nervous system of mice and rats, as studied with the electron microscope. J Biophys Biochem Cytol 2, 777-784.

Machesky, L.M., and Hall, A. (1997). Role of actin polymerization and adhesion to extracellular matrix in Rac- and Rho-induced cytoskeletal reorganization. J Cell Biol 138, 913-926.

Makinodan, M., Rosen, K.M., Ito, S., and Corfas, G. (2012). A critical period for social experiencedependent oligodendrocyte maturation and myelination. Science 337, 1357-1360.

Manning, B.D., and Cantley, L.C. (2007). AKT/PKB signaling: navigating downstream. Cell 129, 1261-1274.

Martin-Belmonte, F., and Rodriguez-Fraticelli, A.E. (2009). Chapter 3: acquisition of membrane polarity in epithelial tube formation patterns, signaling pathways, molecular mechanisms, and disease. International review of cell and molecular biology 274, 129-182.

Matheson, D.F. (1970). Some quantitative aspects of myelination of the optic nerve in rat. Brain Res 24, 257-269.

Matthews, M.A., and Duncan, D. (1971). A quantitative study of morphological changes accompanying the initiation and progress of myelin production in the dorsal funiculus of the rat spinal cord. J Comp Neurol 142, 1-22.

Maturana, H.R. (1960). The fine anatomy of the optic nerve of anurans--an electron microscope study. J Biophys Biochem Cytol 7, 107-120.

Mirsky, R., Stewart, H.J., Tabernero, A., Bradke, F., Brennan, A., Dong, Z., and Jessen, K.R. (1996). Development and differentiation of Schwann cells. Rev Neurol (Paris) 152, 308-313.

Mobius, W., Cooper, B., Kaufmann, W.A., Imig, C., Ruhwedel, T., Snaidero, N., Saab, A.S., and Varoqueaux, F. (2010). Electron microscopy of the mouse central nervous system. Methods Cell Biol 96, 475-512.

Moore, C.L., Kalil, R., and Richards, W. (1976). Development of myelination in optic tract of the cat. J Comp Neurol 165, 125-136.

Narayanan, S.P., Flores, A.I., Wang, F., and Macklin, W.B. (2009). Akt signals through the mammalian target of rapamycin pathway to regulate CNS myelination. J Neurosci $29,6860-6870$.

Nave, K.A. (2010). Myelination and the trophic support of long axons. Nature reviews Neuroscience 11, 275-283.

Nave, K.A., and Trapp, B.D. (2008). Axon-glial signaling and the glial support of axon function. Annu Rev Neurosci 31, 535-561.

Nobes, C.D., and Hall, A. (1995). Rho, rac, and cdc42 GTPases regulate the assembly of multimolecular focal complexes associated with actin stress fibers, lamellipodia, and filopodia. Cell 81, 53-62. 
Parazzini, C., Baldoli, C., Scotti, G., and Triulzi, F. (2002). Terminal zones of myelination: MR evaluation of children aged 20-40 months. AJNR American journal of neuroradiology 23, 1669-1673.

Pedraza, L., and Colman, D.R. (2000). Fluorescent myelin proteins provide new tools to study the myelination process. J Neurosci Res 60, 697-703.

Pedraza, L., Huang, J.K., and Colman, D. (2009). Disposition of axonal caspr with respect to glial cell membranes: Implications for the process of myelination. J Neurosci Res 87, 3480-3491.

Pereira, G.B., Meng, F., Kockara, N.T., Yang, B., and Wight, P.A. (2013). Targeted deletion of the antisilencer/enhancer (ASE) element from intron 1 of the myelin proteolipid protein gene (PIp1) in mouse reveals that the element is dispensable for Plp1 expression in brain during development and remyelination. J Neurochem 124, 454-465.

Peters, A. (1960a). The formation and structure of myelin sheaths in the central nervous system. J Biophys Biochem Cytol 8, 431-446.

Peters, A. (1960b). The structure of myelin sheaths in the central nervous system of Xenopus laevis (Daudin). J Biophys Biochem Cytol 7, 121-126.

Peters, A. (1962). Plasma membrane contacts in the central nervous system. J Anat 96, 237-248.

Peters, A. (1964). Observations on the Connexions between Myelin Sheaths and Glial Cells in the Optic Nerves of Young Rats. J Anat 98, 125-134.

Peters, A. (1966). The node of Ranvier in the central nervous system. Quarterly journal of experimental physiology and cognate medical sciences 51, 229-236.

Peters, A., and Muir, A.R. (1959). The relationship between axons and Schwann cells during development of peripheral nerves in the rat. Quarterly journal of experimental physiology and cognate medical sciences $44,117-130$.

Peters, A., and Proskauer, C. C. (1969). "The Structure and Function of Nervous Tissue" (G. H. Bourne, ed.), Vol. 1, pp. 141-186. Academic Press, New York.

Quarles, R.H. (2002). Myelin sheaths: glycoproteins involved in their formation, maintenance and degeneration. Cell Mol Life Sci 59, 1851-1871.

Quarles R., Macklin W., Morell. P., (2006). Myelin formation, Structure and biochemistry. Basic Neurochemistry: Molecular, cellular and medical aspects. 0-12-088397-X

Ransom, B.R., Butt, A.M., and Black, J.A. (1991). Ultrastructural identification of HRP-injected oligodendrocytes in the intact rat optic nerve. Glia 4, 37-45.

Remahl, S., and Hildebrand, C. (1990). Relations between axons and oligodendroglial cells during initial myelination. II. The individual axon. J Neurocytol 19, 883-898.

Remahl, S., and Hilderbrand, C. (1990). Relation between axons and oligodendroglial cells during initial myelination. I. The glial unit. J Neurocytol 19, 313-328.

Richards, W., Kalil, R., and Moore, C.L. (1983). An observation about myelination. Experimental brain research 52, 219-225. 
Ridley, A.J. (2011). Life at the leading edge. Cell 145, 1012-1022.

Ridley, A.J., Paterson, H.F., Johnston, C.L., Diekmann, D., and Hall, A. (1992). The small GTP-binding protein rac regulates growth factor-induced membrane ruffling. Cell 70, 401-410.

Robertson, J.D. (1955). The ultrastructure of adult vertebrate peripheral myelinated nerve fibers in relation to myelinogenesis. J Biophys Biochem Cytol 1, 271-278.

Rosenberg, S.S., Ng, B.K., and Chan, J.R. (2006). The quest for remyelination: a new role for neurotrophins and their receptors. Brain Pathol 16, 288-294.

Rosenbluth, J. (1966). Redundant myelin sheaths and other ultrastructural features of the toad cerebellum. J Cell Biol 28, 73-93.

Rushton, W.A. (1951). A theory of the effects of fibre size in medullated nerve. J Physiol 115, 101-122.

Saab, A.S., Tzvetanova, I.D., and Nave, K.A. (2013). The role of myelin and oligodendrocytes in axonal energy metabolism. Curr Opin Neurobiol 23, 1065-1072.

Shen, X.Y., Billings-Gagliardi, S., Sidman, R.L., and Wolf, M.K. (1985). Myelin deficient (shimld) mutant allele: morphological comparison with shiverer (shi) allele on a B6C3 mouse stock. Brain research 360, 235-247.

Sherman, D.L., and Brophy, P.J. (2005). Mechanisms of axon ensheathment and myelin growth. Nat Rev Neurosci 6, 683-690.

Sobottka, B., Ziegler, U., Kaech, A., Becher, B., and Goebels, N. (2011). CNS live imaging reveals a new mechanism of myelination: the liquid croissant model. Glia 59, 1841-1849.

Tai, G., Reid, B., Cao, L., and Zhao, M. (2009). Electrotaxis and wound healing: experimental methods to study electric fields as a directional signal for cell migration. Methods Mol Biol 571, 77-97.

Tauber, H., Waehneldt, T.V., and Neuhoff, V. (1980). Myelination in rabbit optic nerves is accelerated by artificial eye opening. Neurosci Lett 16, 235-238.

Taveggia, C., Feltri, M.L., and Wrabetz, L. (2010). Signals to promote myelin formation and repair. Nat Rev Neurol 6, 276-287.

Thurnherr, T., Benninger, Y., Wu, X., Chrostek, A., Krause, S.M., Nave, K.A., Franklin, R.J., Brakebusch, C., Suter, U., and Relvas, J.B. (2006). Cdc42 and Rac1 signaling are both required for and act synergistically in the correct formation of myelin sheaths in the CNS. J Neurosci 26, 10110-10119.

Trajkovic, K., Dhaunchak, A.S., Goncalves, J.T., Wenzel, D., Schneider, A., Bunt, G., Nave, K.A., and Simons, M. (2006). Neuron to glia signaling triggers myelin membrane exocytosis from endosomal storage sites. J Cell Biol 172, 937-948.

Trapp, B.D., Andrews, S.B., Wong, A., O'Connell, M., and Griffin, J.W. (1989). Co-localization of the myelin-associated glycoprotein and the microfilament components, F-actin and spectrin, in Schwann cells of myelinated nerve fibres. J Neurocytol 18, 47-60.

Trapp, B.D., Moench, T., Pulley, M., Barbosa, E., Tennekoon, G., and Griffin, J. (1987). Spatial segregation of mRNA encoding myelin-specific proteins. Proc Natl Acad Sci U S A 84, 7773-7777. 
Vincze, A., Mazlo, M., Seress, L., Komoly, S., and Abraham, H. (2008). A correlative light and electron microscopic study of postnatal myelination in the murine corpus callosum. Int J Dev Neurosci 26, 575584.

Wake, H., Lee, P.R., and Fields, R.D. (2011). Control of local protein synthesis and initial events in myelination by action potentials. Science 333, 1647-1651.

Waxman S., Kocsis J., Stys P., (1995). The axon. Orford Uni Press.

Webster, H.D. (1971). The geometry of peripheral myelin sheaths during their formation and growth in rat sciatic nerves. J Cell Biol 48, 348-367.

Werner, H.B., Kuhlmann, K., Shen, S., Uecker, M., Schardt, A., Dimova, K., Orfaniotou, F., Dhaunchak, A., Brinkmann, B.G., Mobius, W., et al. (2007). Proteolipid protein is required for transport of sirtuin 2 into CNS myelin. J Neurosci 27, 7717-7730.

Wiggins, R.C., Chongjie, G., Delaney, C., and Samorajski, T. (1988). Development of axonaloligodendroglial relationships and junctions during myelination of the optic nerve. Int J Dev Neurosci 6, 233-243.

Wullschleger, S., Loewith, R., and Hall, M.N. (2006). TOR signaling in growth and metabolism. Cell 124, 471-484.

Yang, S., Li, C., Qiu, X., Zhang, L., Lu, W., Chen, L., Zhao, Y.Y., Shi, X.Y., Huang, C.X., Cheng, G.H., et al. (2013). Effects of an enriched environment on myelin sheaths in the white matter of rats during normal aging: A stereological study. Neuroscience 234, 13-21.

Yin, X., Peterson, J., Gravel, M., Braun, P.E., and Trapp, B.D. (1997). CNP overexpression induces aberrant oligodendrocyte membranes and inhibits MBP accumulation and myelin compaction. J Neurosci Res 50, 238-247.

Zalc, B. (2006). The acquisition of myelin: a success story. Novartis Foundation symposium 276, 15-21; discussion 21-15, 54-17, 275-281.

Zhao, M., Song, B., Pu, J., Wada, T., Reid, B., Tai, G., Wang, F., Guo, A., Walczysko, P., Gu, Y., et al. (2006). Electrical signals control wound healing through phosphatidylinositol-3-OH kinase-gamma and PTEN. Nature 442, 457-460. 


\section{Curriculum Vitae}

\section{Nicolas SNAIDERO}

Date of Birth: 18/05/1984

\section{Adress}

Ulmenweg $2 b$

37077 Goettingen

Germany

LinkedIn profile:
Nationality: French

Mobile:+49-(0)176-283-68170

Email: nicolas.snaidero@free.fr snaidero@em.mpg.de

http://www.linkedin.com/profile/view?id=136228807

\section{SKILLS \& EXPERTISE}

Personal aptitudes for:

oProject management during Ph.D and collaboration projects

oInternationalcommunication/ team work

oResponsibility/ organization in representative functions and conferences organization

-Communicational skills by giving lectures, workshops, student courses, seminars and conferences

\section{Microscopy techniques:}

oTransmissionElectron microscopy/ Tomography

-Confocal microscopy

oScanning Electron Microscopy: FIBSEM

-Atomic force microscopy

\section{Sample preparation:}

oHigh pressure freezing olmmunoEM, sectioning (Tokuyasu) oLaser micro dissection

oPerfusion and dissection oSlice and cell culture

Data acquisition and analysis:

olmod/ 3D modeling

olmage J/ Adobe suite

oCoreldraw

Generic:

oMouse anatomy

○Cell morphology

oMolecular biology methods

Languages:

oFrench (native)

proficiency)

○English (bilingual proficiency)

oGerman (limited working

\section{EDUCATION}

Second year of MSc and currently Ph.D in Neuroscience program, Georg-AugustUniversitätGöttingen, International Max Plank Research school (IMPRS) (2008-present)

First year of MSc Neuroscience, University of Glasgow (2007-2008)

Bachelor of Bio-informatics and Biology, Joseph Fourier, Grenoble, France (2004-2007)

Medical studies, Grenoble, France (2002-2004) 


\section{LABORATORY and RESEARCH EXPERIENCE}

Ph.D: Morphological and biochemical investigation of the myelination process in CNS

- Advanced morphological investigation of the myelination process in mammalians using state of the art imaging techniques: HPF-TEM, Focus ion beam milling, 3D reconstruction and live imaging

- Understanding of the molecular mechanisms that are underlying the myelination process in CNS

- Characterization of the causes of abnormal myelin growth leading to myelin outfoldings in neurological diseases.

(08/10-present) Supervisor Prof Mikael Simons: MPI EM, Göttingen, Germany

\section{Master: Exploring techniques for 3D electron microscopy studies on myelination}

- Assessment of multiple high resolution volume microscopy techniques and 3D reconstruction suitable for nervous system morphological analysis: serial sectioning, tomography and focus ion beam milling

- Participation in the establishing of high pressure freezing and freeze substitution in the institute

(01/10-06/10) Supervisor Dr Wiebke Möbius:MPI EM, Göttingen, Germany

Bachelor: Biochemical investigation of axonal transport regulators in myelin mutants

- Investigation of axonal transport impairment in the spastic paraplegia type 2 neuropathy mouse model (Plp1ko) and in Cnp1 ko

- Molecular and morphological analysis showed that similar degeneration pathology is mediated by different molecular mechanisms in Plp1 and Cnp1ko mouse models

(09/07-05/08) Supervisor Dr Julia Edgar:AppliedNeurobiology Group, University of Glasgow UK

\section{HONORS, CONFERENCE ORGANISATION and INTERESTS}

Representative functions:

(2005-2006) At Université Joseph Fourier (Student representative)

(2012-2013) At Max-Planck-Institute fürExperimentelleMedizin(PhD representative)

\section{Honors:}

(2007-2008) Erasmus scholarship at Glasgow University

(2008-2009) Stipend of the Excellence Foundation for the promotion of the Max Planck Society

Organisation of conferences:

Neurizons 2011 From Molecules to Mind, Making sense of the brain (international conference)

PANOS 2012 Light meets Electron Microscopy (international meeting)

Interests:

-Modelling and painting on miniature models (Warhammer $\left.{ }^{\circledR}\right)$

-Team sport such as rowing (part of an 8+ for 2 years) or volleyball (University teams of Grenoble and Glasgow)

-Real time strategy team games such as Starcraft $\circledast$ 


\section{List of publication}

Myelin Membrane Wrapping of CNS Axons by PI(3,4,5)P3-Dependent Polarized Growth at the Inner Tongue

Snaidero N, Moebius W, Czopka T,HekkingL.,Mathisen C,Verkleij D,Goebbels S, Edgar J,Merkler D, Lyons D, Nave K,Simons M.

Cell. 2014 Jan;

A Global In Vivo Drosophila RNAi Screen Identifies a Key Role of CeramidePhosphoethanolamine for Glial Ensheathment of Axons.

Ghosh A, Kling T, Snaidero N, Sampaio JL, Shevchenko A, Gras H, Geurten B, Göpfert MC, Schulz JB, Voigt A, Simons M.

PLoS Genet. 2013 Dec;9(12):e1003980.

Cryo FIB-SEM: volume imaging of cellular ultrastructure in native frozen specimens. Schertel A*, Snaidero N*, Han HM, Ruhwedel T, Laue M, Grabenbauer M, Möbius W. J Struct Biol. 2013 Nov;184(2):355-60.

*Equal contribution

Choroid plexus transcytosis and exosome shuttling deliver folate into brain parenchyma. Grapp M, Wrede A, Schweizer M, Hüwel S, Galla HJ, Snaidero N, Simons M, Bückers J, Low PS, Urlaub H, Gärtner J, Steinfeld R.

Nat Commun. 2013;4:2123.

Myelin membrane assembly is driven by a phase transition of myelin basic proteins into a cohesive protein meshwork.

Aggarwal S, Snaidero N, Pähler G, Frey S, Sánchez P, Zweckstetter M, Janshoff A, Schneider A, Weil MT, Schaap IA, Görlich D, Simons M.

PLoS Biol. 2013;11(6).

Loss of electrostatic cell-surface repulsion mediates myelin membrane adhesion and compaction in the central nervous system.

Bakhti M, Snaidero N, Schneider D, Aggarwal S, Möbius W, Janshoff A, Eckhardt M, Nave KA, Simons $M$.

ProcNatlAcadSci U S A. 2013 Feb 19;110(8)

The histone H2B monoubiquitination regulatory pathway is required for differentiation of multipotent stem cells.

Karpiuk O, Najafova Z, Kramer F, Hennion M, Galonska C, König A, Snaidero N, Vogel T, Shchebet A, Begus-Nahrmann Y, Kassem M, Simons M, Shcherbata H, Beissbarth T, Johnsen SA. Mol Cell. 2012 Jun 8;46(5):705-13. 
Cell polarity in myelinating glia: from membrane flow to diffusion barriers.

Simons M, Snaidero N, Aggarwal S.

BiochimBiophysActa. 2012 Aug;1821(8):1146-53.

A size barrier limits protein diffusion at the cell surface to generate lipid-rich myelin-membrane sheets.

Aggarwal S, Yurlova L, Snaidero N, Reetz C, Frey S, Zimmermann J, Pähler G, Janshoff A, Friedrichs J, Müller DJ, Goebel C, Simons M.

Dev Cell. 2011 Sep 13;21(3):445-56.

Electron microscopy of the mouse central nervous system. Möbius W, Cooper B, Kaufmann WA, Imig C, Ruhwedel T, Snaidero N, Saab AS, Varoqueaux F. Methods Cell Biol. 2010;96:475-512.

Early ultrastructural defects of axons and axon-glia junctions in mice lacking expression of Cnp1. Edgar JM, McLaughlin M, Werner HB, McCulloch MC, Barrie JA, Brown A, Faichney AB, Snaidero N, Nave KA, Griffiths IR.

Glia. 2009 Dec;57(16):1815-24. 